\title{
A Mathematical Principle of Art and Human Vision
}

\author{
Xin Zhang ${ }^{1^{*}}$
}

\begin{abstract}
The study of visual illusions is an old subject and an important part of the psychology of human visual perception, but hitherto there has been no single principle able to explain radically different kinds of visual illusions conjointly. Such a principle does exist, as is to be shown, and has the virtue of being rigorous: it is the mathematical theory of Fourier analysis. A great many visual illusions are what happen when the visual objects involved undergo certain frequency filtering, a concept deduced from Fourier analysis. Phenomena thus explained belong in these distinct categories: brightness illusions, colour illusions, geometrical illusions, and motion illusions, all of which have been simulated with computer programmes based on this mathematical principle. Visual illusions obeying this principle have in fact been depicted in Western painting for centuries, and art can in certain ways shed light on the quest for the understanding of human vision.
\end{abstract}

The fundamental laws of nature known to us through the discipline of physics, governing all physical phenomena and also serving as explanations of them, are expressed in the exact language of mathematics. It is therefore unsurprising to wonder whether that is true within an even broader scope. Where the human mind is in any way concerned, however, a contrast is conspicuous: that in such domains as psychology and fine art, mathematics has up till now played no significant part in helping us unravel mysteries.

Yet facts to be presented below suggest that mathematical principles do exist in human visual perception, and in fine art as well. To be more exact, one mathematical theory, Fourier analysis, is the key to delineating a large number of visual illusions (also called optical illusions) of different kinds, and also certain characteristics of Western painting. Those visual illusions involve both static images and animated ones; static image illusions, including geometrical ones induced by lines or shapes, are the main focus of study in this paper.

Psychologists were not, strictly speaking, the first group of people in human history to have studied visual illusions as a professional activity; those who did it first, for several hundred years, but practically unnoticed by all, were artists, many of whom consistently depicted the visual illusions observed by themselves onto their works of art. For instance, of great relevance to brightness illusions and colour illusions are painters in oil in Europe after the Renaissance. Examples will be given.

In this paper, the concept of a visual illusion has a slightly narrower sense than usual. It is restricted to the visual system's planar alteration or distortion of the visual scene, and does not include, among others, the illusion, in fine art, of volume and space (i.e. of threedimensionality) created on the flat surface of a painting by the use of perspective or light and shade. A little more clarification follows. The visual scene in front of an eye (that is, one eye),

\footnotetext{
${ }^{1}$ In Luoyang, Henan province, China, and currently not affiliated to any university or research institute. The author used to specialize in physics at Tsinghua University, Beijing, China.

*e-mail: zhangxin6phy@foxmail.com
} 
whether a real three-dimensional scene or just a two-dimensional image to be observed, is always equivalent to a two-dimensional image there (to put it crudely, the three-dimensional scene can be projected onto a vertical plane in front of the eye, thereby becoming a twodimensional image). It is the monochromatic, chromatic, or geometric distortion by the visual system of this two-dimensional image into a different two-dimensional image that is considered as an illusion and is studied in this paper. That other kind of illusion just now mentioned, on the other hand, involves no such distortion, but exists as the brain's special interpretation of an image, and so is irrelevant here.

Furthermore it is worth noting that the two fundamental forms of physical existence in the universe, waves and particles, complementary to each other, appear in certain respects to have direct counterparts in the workings of human vision.

The basic ideas of the branch of mathematics called Fourier analysis - or in a less complicated but more practical form, Fourier series - are familiar to all those with a background in the physical sciences, engineering, or mathematics, but may well sound foreign to those with a background in the social sciences or biology. So a very brief, intuitive description of what this part of mathematics is about had better be presented, without mathematical rigour, for the latter group, hence the section Fourier Analysis, which immediately follows; but readers belonging to the former group may want to skip it and go on to the section next to it, Visual filtering.

\section{Fourier analysis}

Central to Fourier analysis is the concept of decomposing a periodic function into simple sine waves the sum of which equals that function. A simplistic illustration of this function decomposition is given in Fig. 1, where the frames, identical in size, act as coordinate axes for the curves enclosed in them. Here we have a special periodic function (shown in the top left frame, whose left and right borders enclose one period of the function precisely) so constructed that it is able to be decomposed exactly into six sine waves of different frequencies, magnitudes, and phases (the $n$ beneath each frame is the number of that wave's periods per frame), plus one constant function $(n=0)$. Summation of these seven waves $(n=0,1,2, \ldots, 6)$ produces the original function. 


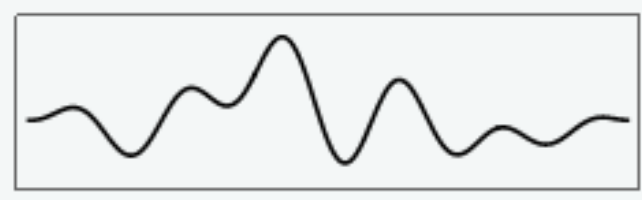

original function

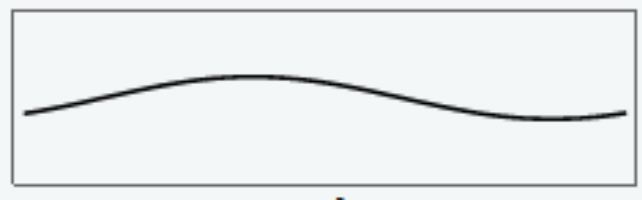

$n=1$

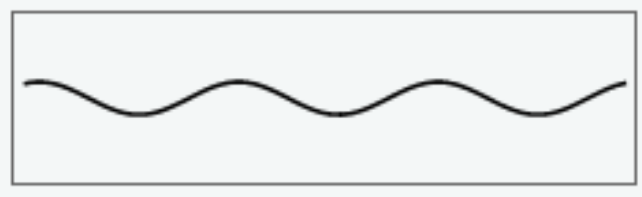

$n=3$

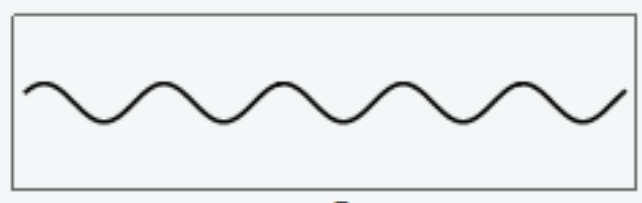

$n=5$
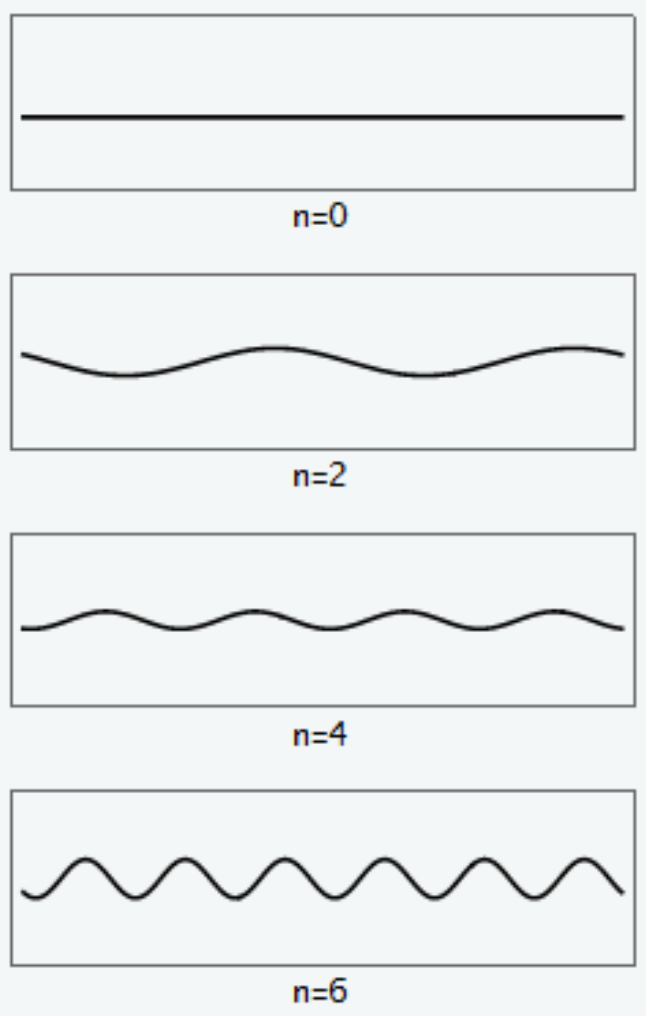

Figure 1

Fourier analysis shows that an arbitrary periodic function $f(x)$ (herein we are concerned only with periodic functions) may be decomposed into many, generally an infinite number of, simple sinusoids (namely, sinusoidal waves) of different frequencies, amplitudes, and phases; the sum of these sinusoids, called its Fourier series, equals $f(x)$, except perhaps at certain peculiar positions, if any. This process is called Fourier transformation; the coefficients of these sinusoids, $f$ s Fourier transform.

Fully analogous to the above case in one dimension, an arbitrary two dimensional periodic function $g(x, y)$, according to Fourier analysis, can be decomposed into a series of two dimensional sinusoidal waves of different frequencies, magnitudes, and phases. Furthermore, as a two dimensional object, a black-and-white image, mathematically speaking, is a two dimensional function; if the image is indefinitely repeated horizontally and vertically, it becomes a two dimensional periodic function $g(x, y)$ as just mentioned, for which, at a given position $\left(x_{0}, y_{0}\right)$ on the image, $g\left(x_{0}, y_{0}\right)$ is the brightness value there. Therefore, an arbitrary greyscale image can be decomposed into many two dimensional sinusoidal waves, whose sum, or the superposition of which, is that very image.

Also crucial to the understanding of this paper is the concept of filtering a function $f$ - or, to be more exact, filtering its component sinusoidal waves - to get a new function. In this process, each of $f$ s component waves is multiplied by a number to change its amplitude: namely, the 0 th component is multiplied by $k(0)$, the 1 st component by $k(1)$, the 2 nd by $k(2)$, and so on; so the $n$th component sinusoid is multiplied by $k(n)$, where $n=0,1,2,3 \ldots$, and in general, $0 \leq k(n) \leq 1$; the sum of these modified sinusoids is the new function we want. For instance, if $k(5)=0.2$, then the $5^{\text {th }}$ sinusoid is reduced in amplitude to $0.2=1 / 5$ of its original size. 
As an example, we can filter the function in Fig. 1, which has seven components $(n=0,1,2,3,4,5,6)$, in this way: let the $0^{\text {th }}$ component sinusoid be multiplied by 0.8 , the $1^{\text {st }}$ one by 0.9 , the $2^{\text {nd }}$ by 1 , the $3^{\text {rd }}$ by 0.8 , the $4^{\text {th }}$ by 0.6 , the $5^{\text {th }}$ by 0.2 , and the $6^{\text {th }}$ by 0.1 . The resultant sinusoids are plotted in Fig. 2, each with its frequency number $n$ below it. Then, adding all these together, we get the filtered output function shown in the top left-hand corner of this figure.

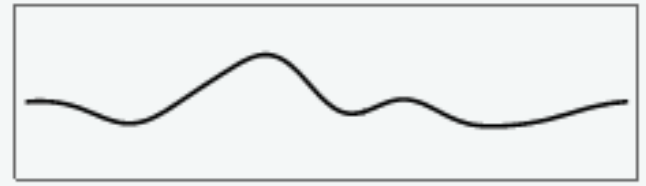

filtered function

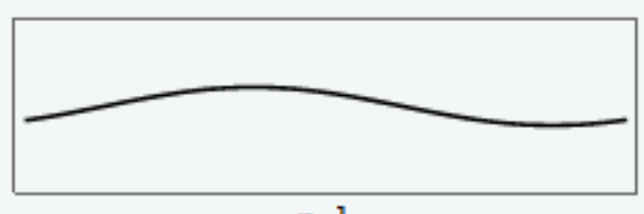

$n=1$

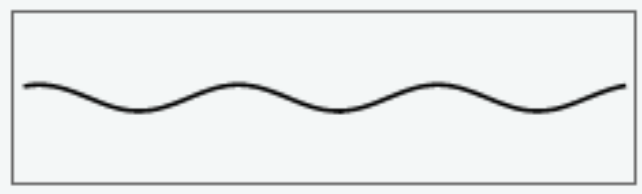

$n=3$

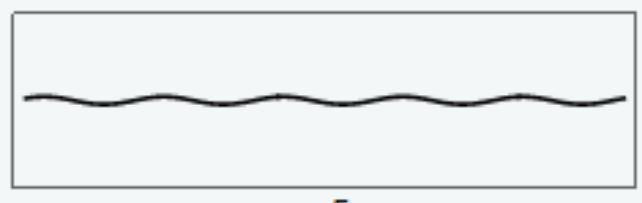

$n=5$

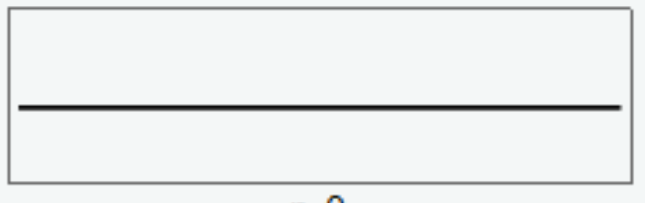

$n=0$

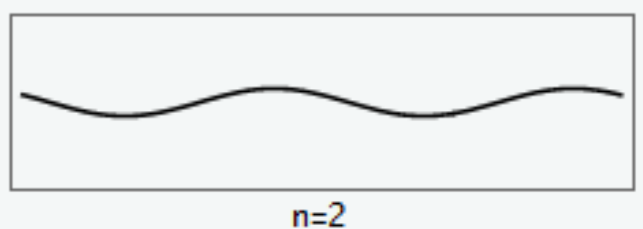

$\mathrm{n}=2$

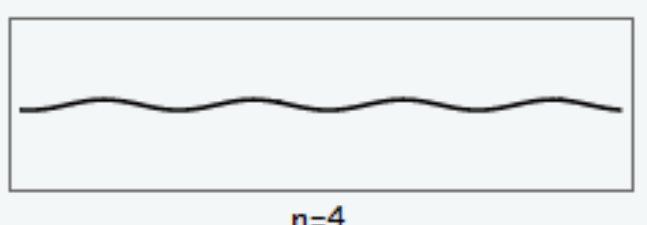

$n=4$

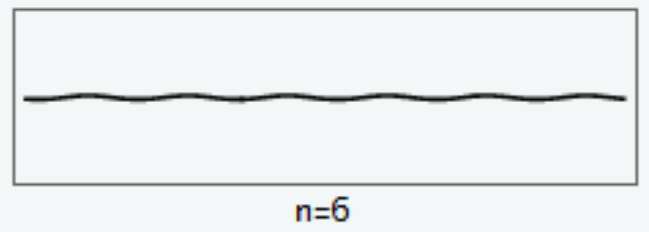

Figure 2

More detailed treatise of this part of knowledge can be found in certain textbooks on digital image processing. In many calculus courses the section on Fourier series is also helpful for a basic understanding of the subject.

In addition, it should be noted here that filtering in the frequency domain of a function is mathematically equivalent to what is called convolution in its space domain, with a corresponding filter or 'mask'.

\section{Visual filtering}

The mathematics discussed above is highly relevant to human visual perception for two reasons, one given by science, and the other by fine art. The former, known through experiments, is that certain cells in the primary visual cortex respond very strongly to sinusoid gratings, as shown in Fig. 4, where the brightness on the image varies as a sinusoidal wave along a certain direction (the number of cycles per unity view angle along that direction being the spatial frequency of the grating). Also, experiments conducted on human subjects ${ }^{1}$ show that the sensitivity of human visual perception to such gratings of different frequencies 
is as plotted schematically in Fig. 3, exhibiting a certain degree of similarity to what are called 'band-pass filtering' and 'low-pass filtering' in the field of signal processing. (If non-linear scales are used for the axes, the shape of the sensitivity curve should look fairly dissimilar from that in Fig. 3.)

This sensitivity curve of the visual system is naturally that of a frequency filter, determining how strong a grating image of a certain frequency - i.e. a sinusoidal wave of that frequency appears to visual perception. A sensitivity of zero for a certain high frequency, for instance, means that a grating of this frequency is totally filtered out by the visual system, and, mathematically, that the amplitude of this grating is multiplied by zero, the value of the filter curve at this point of frequency.

Curious though it may sound, a filter curve of practically the same shape can also be arrived at from one or two basic principles in the art of painting.

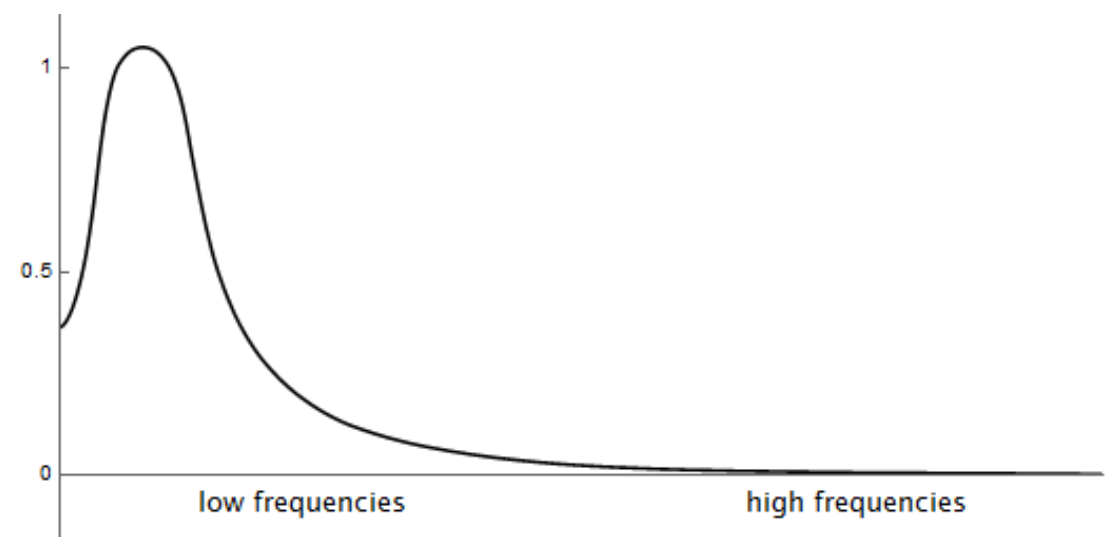

Figure 3

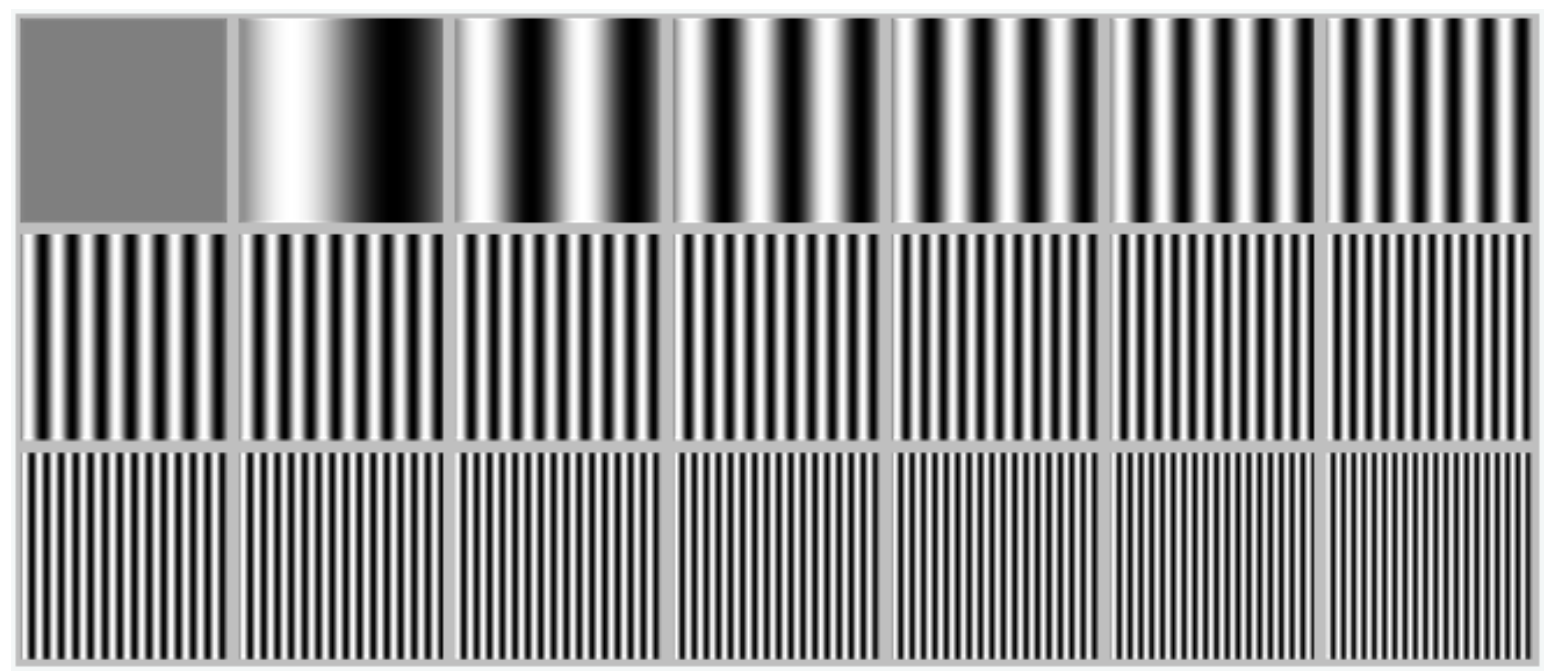

Figure 4

In this connection, two important, opposing concepts in art need to be introduced: unity and variety. Unity signifies that certain formal factors, for instance the colours, in all parts of a painting should look unified and harmonious; variety, that those factors should look varied and different. The aforementioned basic principle is that in a painting it is desirable to have 
as much unity and variety, combined, as possible, with a bias in the best work on the side of unity. Very simply put, the aesthetic aim is variety in unity.

For a basic illustration of the principle, two extreme situations can be considered: if every part of a picture is exactly the same colour, then there is much unity in it, but, owing to a total lack of variety, the picture will be very dull and monotonous; if on the other hand the picture plane is fully covered by patches of randomly chosen raw colours, then in it there is much variety but little or no unity, and as a consequence the picture will look chaotic and unpleasant. What is aesthetically desirable, therefore, is somewhere in between. Such a realization is almost the principle just explained.

In addition to that principle, a special concept needs mention. The word rhythm is not a rare term in discussions of painting or drawing techniques: 'Rhythm ... is where the real mystery and vitality of art exists. ${ }^{2}$ Yet there is something odd about the matter. By rhythm is meant, at least as its most basic sense, a regular repeated pattern; but no painting, not to mention masterpieces, is a regular repeated pattern of any sort. (In Fig. 16, for example, a reproduction of a painting by an old master, by no means is there a regular repeated pattern.) It is therefore in an extremely vague and loose manner that this word is used as a key concept in the principles of fine art; for this very reason the concept is general but mystical, difficult to grasp.

With the notion of Fourier decomposition of a picture and that of visual filtering, however, rhythm and its role in art can be given a much clearer meaning, as follows. The concept of rhythm in art refers to a certain well-organized set of sinusoidal waves (typically twodimensional, but not necessarily so) into which a picture, or a certain formal aspect of it, can be decomposed, each of them being naturally an unambiguously regular repeated pattern. As an overly simplified illustration, the filtered function (only that part in the frame) in the upper left-hand corner of Fig. 2 can be considered. It is, presumably few would disagree, a somewhat agreeable, rhythmical curve, but in it there is no regular repeated pattern, the several 'hills' being very much dissimilar from each other; its components, however, those simple sinusoidal waves in Fig. 2, which when added up form it, are regular repeated patterns each.

On the principle of unity and variety, and on the new concept of rhythm, an analysis of the grating patterns of Fig. 4, leading to a visual filter, is as follows. The analysis as it now is does not sound sufficiently scientific, but this does not matter much, because no other discussions in this paper are logically dependent on it. So the remaining five or six paragraphs in this section, which are devoted to it, can be glanced through as a short diversion.

In Fig. 4 there are 21 images of vertical sinusoid gratings, where the numbers of sinusoid cycles are, respectively, $0,1,2, \ldots, 20$.

Let $v$ represent the number of cycles in a grating. When $v=0$, the image has no rhythm whatever, and no variety, but complete unity; when $v=1$, there is little rhythm in the image, and since the left part is mostly white whereas the right part mostly black, there is too much variety but too little unity; when $v=2$, the presence of rhythm in the images is low, and the image has strong variety but not much unity; when $v=3$, the presence of rhythm is obvious but still not prominent, and the image has more unity than the previous 2 images because there are 3 identical cycles in it; when $v=4,5,6$, the presence of rhythm is prominent and the number of repetitions of black-and-white is comfortable, and the image ranks high in both 
variety and unity; when $7<=v<=15$, although rhythm is prominent, the number of repetitions is making the picture more and more monotonous, i.e., enough of unity but lacking in variety; when $v>=16$, there are so many tiny cycles that the picture looks monotonous again, approaching, in a different fashion, the extreme lack of variety in the $v=0$ case, despite the rhythm it has.

A filter $f l t(n)$ built according to the foregoing analysis ( $n$ here is equivalent to $v$ above), and furthermore to the requirement that its plotted curve should be smooth, will look like the following list (where some high frequency terms, all very small, have been omitted):

$f l t(0)=0.35 ; f l t(1)=0.42 ; f l t(2)=0.58 ; f l t(3)=0.80 ; f l t(4)=0.95 ; f l t(5)=1.00 ; f l t(6)=0.97 ; f l t(7)=0.88$; $f l t(8)=0.76 ; f l t(9)=0.64 ; f l t(10)=0.53 ; f l t(11)=0.43 ; f l t(12)=0.35 ; f l t(13)=0.28 ; \ldots$

Fig. 5 is a plot of this filter.

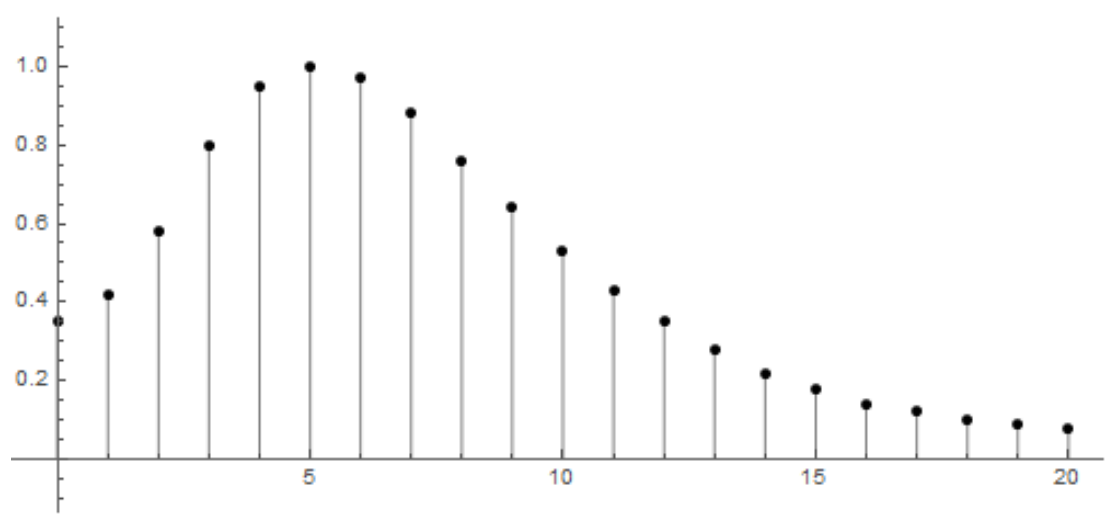

Figure 5

It should be emphasized that this filter was generated solely from aesthetic assessment of those gratings on the principle of unity and variety and the concept of rhythm. Whether for different persons the highest value of $f l t$ is at $n=5$, the overall shape of this curve will not differ much. Further, even if the above aesthetic analysis does not sound scientifically convincing, it does not prevent us from using the filter here described (Fig. 3 and Fig. 5), since there is still that experimentally established sensitivity curve, mentioned earlier, suggesting such a filter, and, far more importantly, since the value of the filter will ultimately be determined by how well it explains the various perplexing visual phenomena.

\section{Extra comments on visual filtering}

In all the discussions that follow, the filtering occurring in human vision, as illustrated in Fig. 3 , that nearly abandons high frequency waves and also reduces waves of other frequencies, especially those of the lowest frequencies, is called Fourier visual filtering, or just visual filtering.

About the shape of the filter function there is one thing to note in particular: the same filter will look different at different image resolutions. For instance, a filter that is defined for all $n<=500$ will look like Fig. 6 at different resolutions (namely, for different domains of $n$ ). 

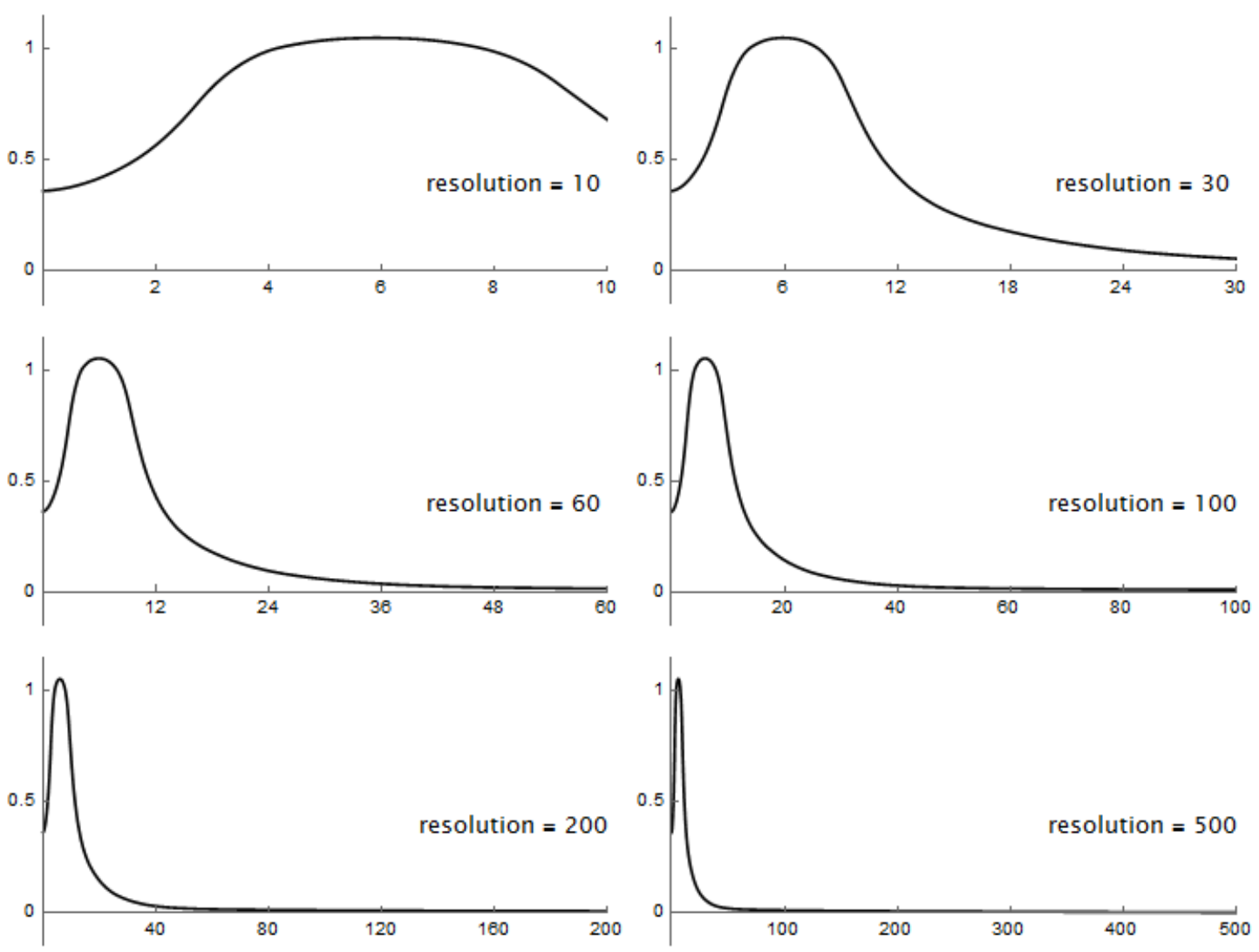

Figure 6

There have been investigations into brightness illusions using the method of convolution of an image, applied with a convolution profile made of two Gaussian functions (one positive and the other negative, and of different shapes and magnitudes) which, when superposed, form an excitatory centre and an inhibitory surround (Fig. 7). This method is able to produce some of the results obtained by the frequency filtering method, because, as mentioned above, filtering in the frequency domain is mathematically equivalent to convolution in the space domain. Nevertheless there are at least two drawbacks of this method to be noted: (a) the convolution profile being made of two Gaussians, this mathematical form is too special and simplistic, and almost certainly will, for that reason, eventually prove to be inadequate to describe myriad human visual phenomena; $(b)$ with such a method, the aesthetic significance of rhythm is barely visible, and creating an acceptable filter through aesthetic principles is impossible. 


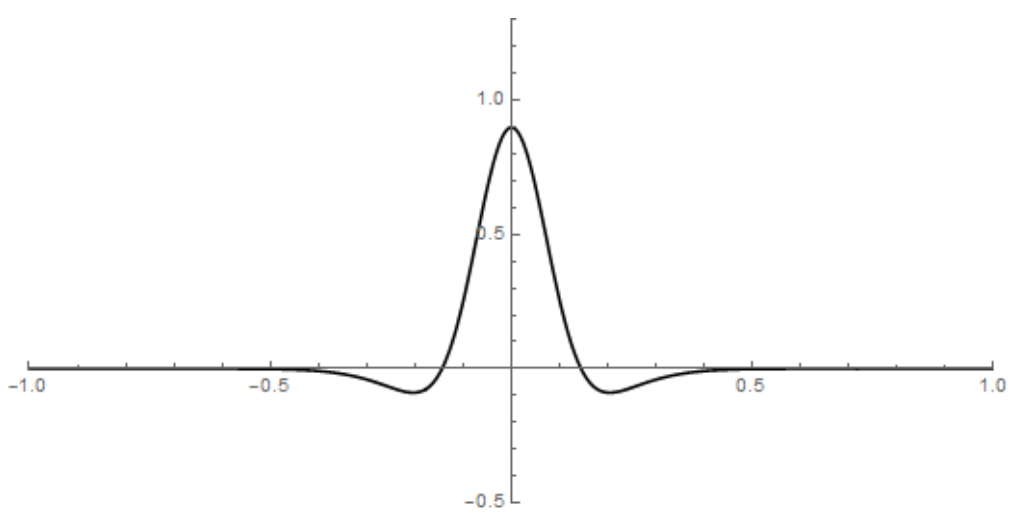

Figure 7

\section{Brightness illusions}

Now that the meaning of visual filtering has been illustrated, it is to be seen how various visual illusions are the results of it. This short section establishes a connection between the monochrome image and mathematics, as a preparation for the modelling of brightness illusions.

In a monochrome image, the darkest and brightest possible colours, namely black and white, can be represented by the numbers 0 and 1, respectively; from black to white there can be different levels of grey of increasing brightness, which can be represented by real numbers between 0 and 1, as shown in Fig. 8, at the centre of which is a monochrome bar of linearly increasing brightness from left to right, the brightness value at a certain point of it being indicated by the number on the axis below it.

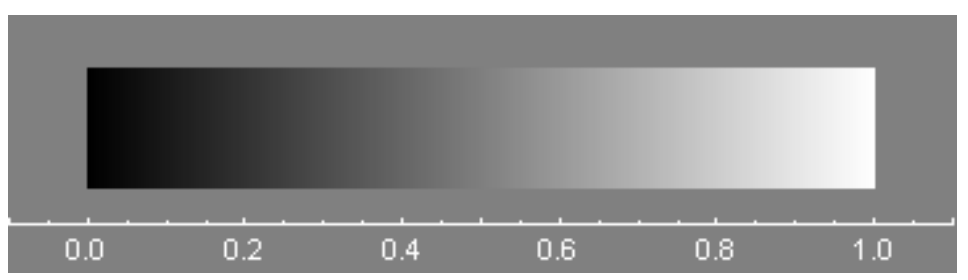

Figure 8

A digital greyscale image that is $m$ pixels in width and $n$ pixels in height ( $m$ and $n$ both positive integers), therefore, is an $m \times n$ matrix whose elements are real numbers larger than or equal to 0 and smaller than or equal to 1 ; so it is also a function $g(i, j)$ taking as arguments $i$ and $j$, the ordered pair of which is the position of a certain pixel. This means the image can be subjected to the visual filtering described in the previous sections.

A note should perhaps be added here: if the reader finds in the ensuing discussions two or three explanations of brightness illusions that are unfortunately not completely unheard in the literature (but were independent of any other's work), please do not stop reading, because they are just a very small part of this paper. 


\section{The generalized Gibbs phenomenon}

The Gibbs phenomenon, in non-rigorous terms, is this. If a periodic function is continuous everywhere except at certain points of discontinuity (where the function has a vertical jump), but its Fourier series is obtained nevertheless, then at those discontinuities, the partial sum of the first $n$ terms of the series, no matter how large $n$ is, will display overshoots, which are non-existent in the original function. (The number $n$ here is a positive integer large enough for the sum to resemble, roughly at least, the original function.)
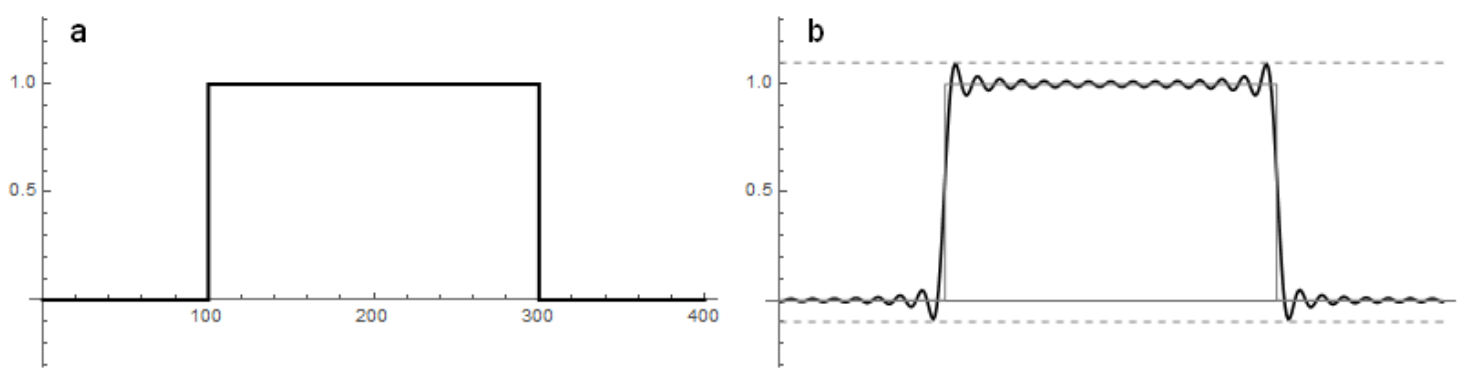

Figure 9

For instance, shown in Fig. 9a is one cycle of a square wave, a periodic function whose value alternates between the level of 0 and that of 1 , the switches themselves being instantaneous; so within this cycle there are two jump discontinuities, or steps, occurring at position 100 of the horizontal axis as well as at 300. After being filtered with all the component waves of frequencies above 30 cut off, and with those of frequencies less than or equal to 30 remaining unchanged, the square wave is turned into the curve presented in Fig. 9b, where up- and down- overshoots are clearly seen at each of the two 'jumps', reaching the dotted horizontal lines, and where the original square wave is also plotted in grey lines for ease of comparison. In addition, ringing, in other words ripples, though nowhere to be found in the original square wave function, is obvious in its truncated Fourier series.

If the filter is not a clear cut-off at a certain frequency, as described above, but a smooth change from 1 to 0 (Fig. 10b), less ringing there will be in the resultant filtered function (Fig. 10a), but still the result of filtering closely resembles the standard Gibbs phenomenon (Fig. $9 \mathrm{~b})$. By the way, the horizontal axis, representing frequencies, of the filter plot in Fig. 10b has been linearly rescaled to run from 0 to 1 (in more detail, with 1 corresponding to half of the diagonal pixel dimension of the image to be filtered); so have those that occur in some subsequent figures. 

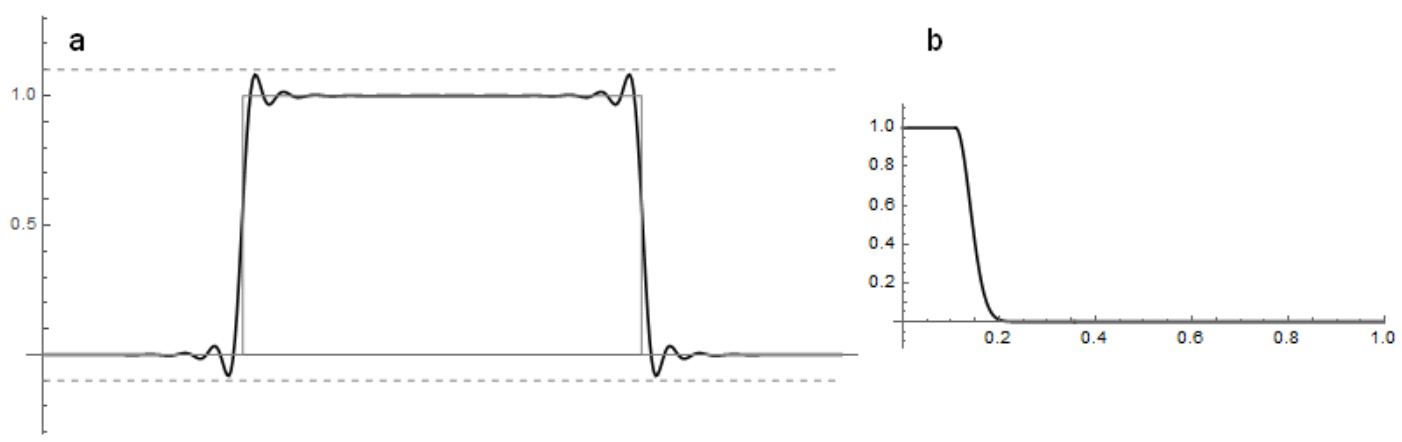

Figure 10

Presented next is the Gibbs phenomenon as occurring in the filtering of images and in brightness illusions.

Fig. 11a consists of vertical black and white bands, and for that reason the greyscale values along any horizontal line on the image is always the square-like function shown in Fig. 9a, no matter where that line is. Filtering this image with the filter in Fig. 11c (which is identical to Fig. 10b) gives us the image in Fig. 11b. Note that, in the filtered image, most parts of the two black bands are no longer completely black (that is, the greyscale values there are now not 0 ) as in the original image, and neither are most parts of the central white band completely white. This corresponds to real human visual experience: if one gazes at the pattern in Fig. 11a long enough, the black areas are no longer totally black any more, except at the borders of it; likewise, the white areas no longer white, except at the boarders. Such a simulation normally requires linearly rescaling the filtered outcome image so that the minimum and maximum of its greyscale values are, as in the original image, 0 and 1, respectively.

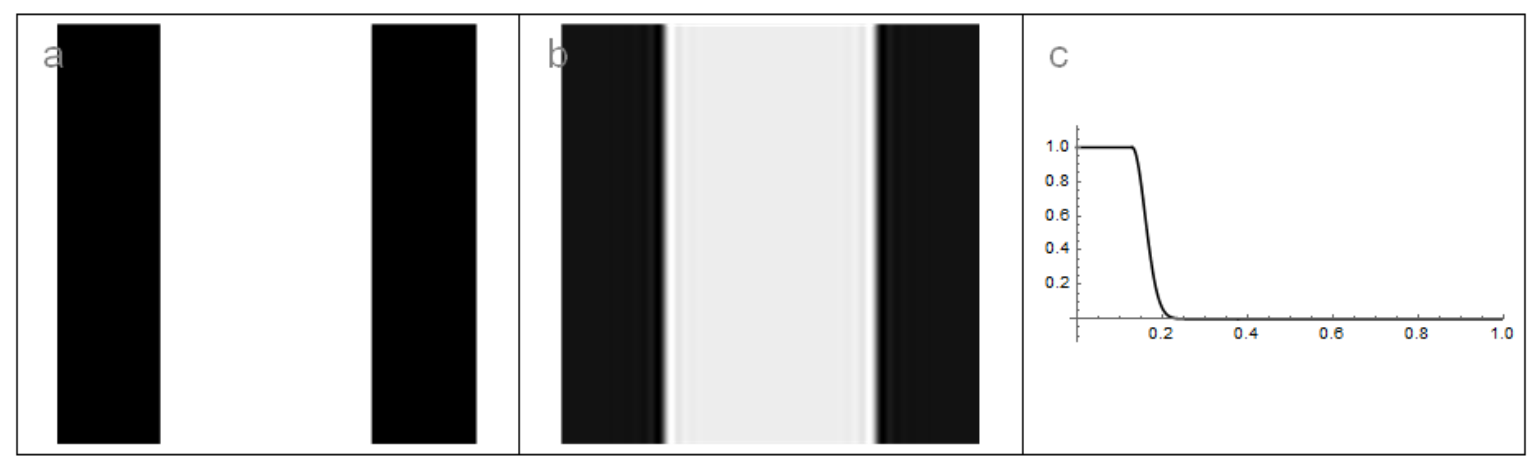

Figure 11

Neither the standard Gibbs phenomenon nor the above filtering outcome, however, is exactly suitable for the investigation of human vision. Instead, the truly relevant thing is what can be called the generalized Gibbs phenomenon, created by filters similar to those in Fig. 3 and Fig. 5, which are evidently different from the low pass filters as seen in the field of signal processing (somewhat similar to that in Fig. 10b). Applying such a filter (Fig. 12b) to the square wave in Fig. 9a, we obtain a result (Fig. 12a) characterized by overshoots that are greater, in this case much greater, than those in the standard Gibbs phenomenon (in Fig. 12a, the two long horizontal dotted lines indicate the upper and lower limits of the overshoots of the standard Gibbs phenomenon, as in Fig. 9b). 


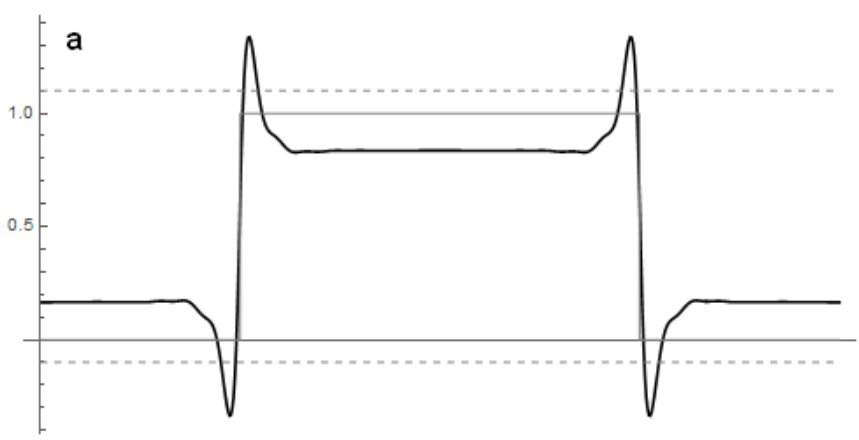

b

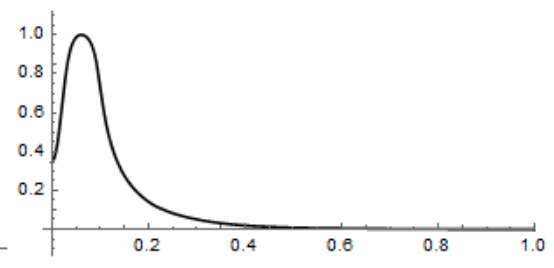

Figure 12

Thus, of the test image in Fig. 11a, a filtering result closer to human visual illusions is as shown in Fig. 13b (it is the second image in the row, the sub-labels $a, b$, and $c$ having been omitted in order not to add distraction to the image when being observed; similar cases of omission will be found later). In order to see the illusion more vividly, one should gaze steadily for at least 10 seconds at a certain position in the image, preferably somewhere near the centre of the white area.

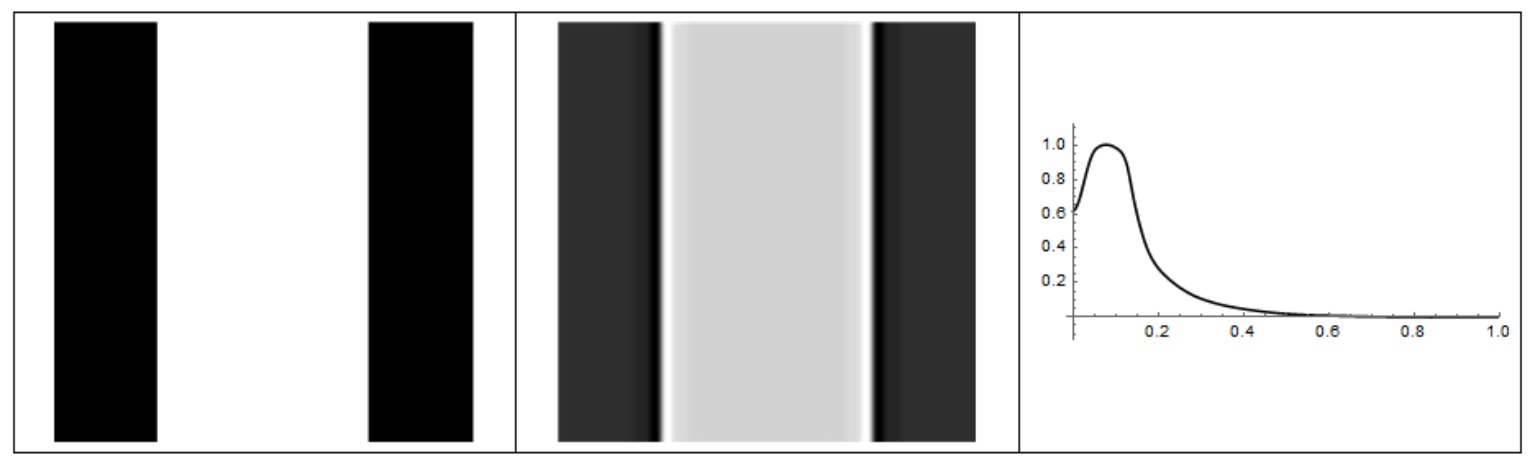

Figure 13

The Mach bands illusion (Fig. 14a, the first panel of Fig. 14, where the label 'a' has been omitted for better viewing of the figure) is also due to the generalized Gibbs phenomenon. In this pattern there are six vertical bands that are increasingly brighter from left to right, and inside each band the brightness is a constant value throughout, though it appears to the human observer that for most of the bands the brightness increases from right to left. Fig. $14 \mathrm{~b}$ is the result of visual filtering of Fig. 14a with the filter in Fig. 14c. (That the actual Mach bands illusion looks much smoother than as shown in Fig. 14b might be an indication that that shape of the filter needs further tweaking, or that another mechanism, similar to what is called the water colour illusion, is involved.) 


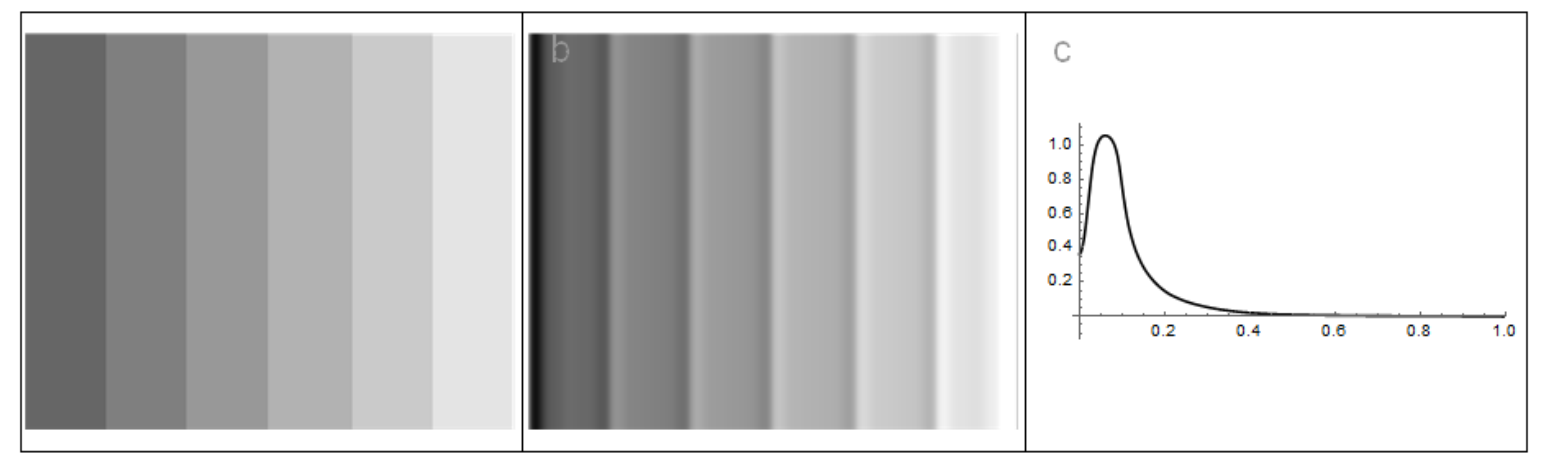

Figure 14

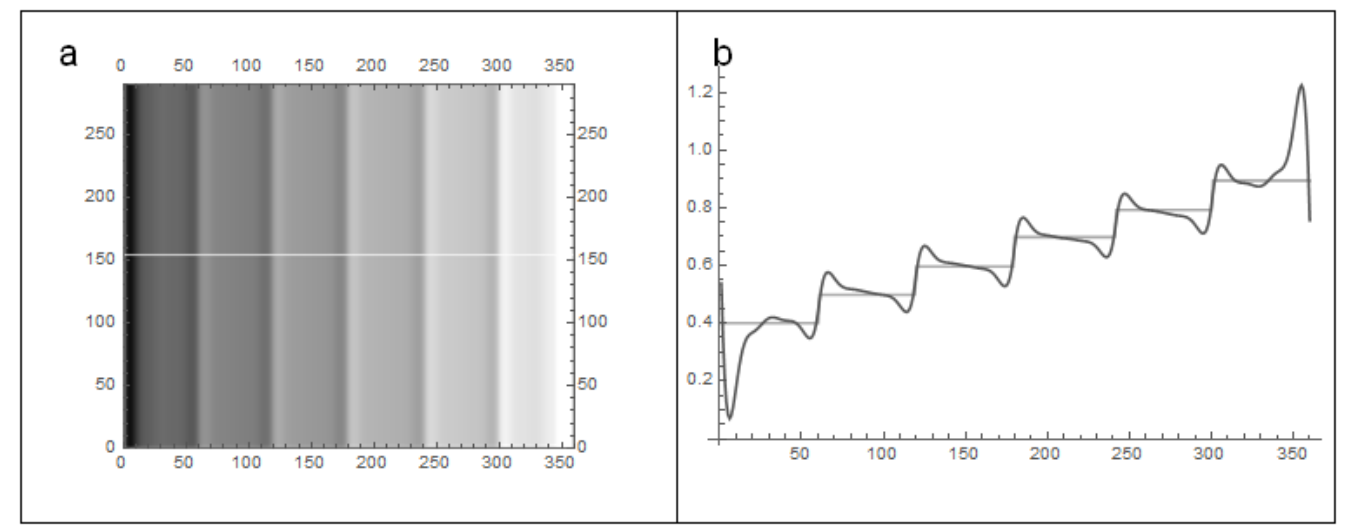

Figure 15

The filtered image in Fig. 14b is duplicated in Fig. 15a, and the grey level values of the filtered image along the white horizontal line in Fig. 15a have been gathered and are plotted as the black curve in Fig. 15b. For comparison, grey level values of points along the same horizontal line in the original unfiltered image are also plotted, namely the grey curve, which looks like a series of stairs. Quantitatively and with complete clarity the plot describes the fact each step of the stairs of brightness, after filtering, is no longer flat, and goes down from left to right: that is to say, each uniform grey band of the original image becomes, after filtering, brighter where it is close to the darker band it touches, and darker where close to the brighter band it touches.

According to the range of visual illusions that can be modelled by Fourier visual filtering, the generalized Gibbs phenomenon is the reason not only for the Mach bands illusion and many other illusions that occur where two different shades or colours are contiguous along a sharp boundary, but also for some important geometrical illusions, some size illusions, and some motion illusions, as will be shown in later discussions.

It is therefore more appropriate to adopt for all these related illusions a common name: illusions of contiguity.

\section{The Gibbs phenomenon as a good thing}


Scientists and engineers have always regarded the Gibbs phenomenon as undesirable artefacts (in the field of signal processing, for instance), hence the development of special methods to eliminate it. Painters, however, working in an entirely different world, have depicted the generalized Gibbs phenomenon - the contiguity illusion - in their paintings for several hundred years: in the trained eye of a traditional artist, enhancing the contrast at the boundary of two parts of different colours, which is the artistic representation of the generalized Gibbs phenomenon, is often aesthetically necessary.

Additionally, whenever the word artist appears in this paper, it is always used in its traditional sense, denoting, typically, a painter that paints on canvases with careful attention to the aesthetic value of his works, realistic or not. (The scholarly-sounding term of aesthetic or any of its derivatives may not often be used by painters, who have more specialized vocabulary to talk about their work and that of others.)

Fig. 16 is a monochrome reproduction of the oil painting Girl with Racket and Shuttlecock, by the 18th-century French painter Jean-Baptiste-Siméon Chardin, one of those masters whom few histories of Western painting would ignore. In this work, various instances of the contiguity illusion can be found, of which the most conspicuous are designated by arrows and marked A, B, C, D, and E in Fig. 17.

It is interesting to note that while $\mathrm{A}, \mathrm{B}, \mathrm{D}$, and $\mathrm{E}$ are all normal depiction of the contiguity illusion, $\mathrm{C}$ is not. This part is particularly noteworthy in that the thin black band (the rim of the dark garment) and the thin white band (the rim of the white underwear or whatever) together form the bottom and top overshoots, respectively, of the generalized Gibbs phenomenon at the dark-garment-and-grey-skin boundary. Painted so dark was the rim of the garment that it is apparent that the painter did it on purpose. Thus, what happens at part $\mathrm{C}$ shows this, that careful arrangement of certain real world items within the painting, in other words some special composition, has been used to accommodate the need for the contiguity illusion.

In addition to the aforementioned ones, there are still other instances of the contiguity illusion, which the interested reader can try to find.

Yet artists would not depict the contiguity illusion at all places where it is logically possible. One of the reasons is this: if they did that, the picture might be flooded with this effect and thus look monotonous and therefore rather unpleasant - in more philosophical terms, that would be a neglect of variety in the aesthetic principle of unity and variety, previously mentioned in the section about visual filtering. Another reason, presumably more important, is that the darkening or brightening effect around the boarder can often spread out on its own side, as the ink dropped on the margin of a basin of water will spread out and finally dye the whole basin of water more or less homogeneously; perhaps it has the same mechanism as the watercolour illusion. The third one is more dependent on the judgement by the trained eye of the painter. It is very likely that this effect, if depicted at curtain places on the painting, will improve how the painting looks, but, if depicted at others, will unfortunately impair that. As a result, it should only be applied where it is indeed needed. Last but not least, the style of an artist also decides how much this illusion should be represented on the canvas; some prefer more of it while others less. 


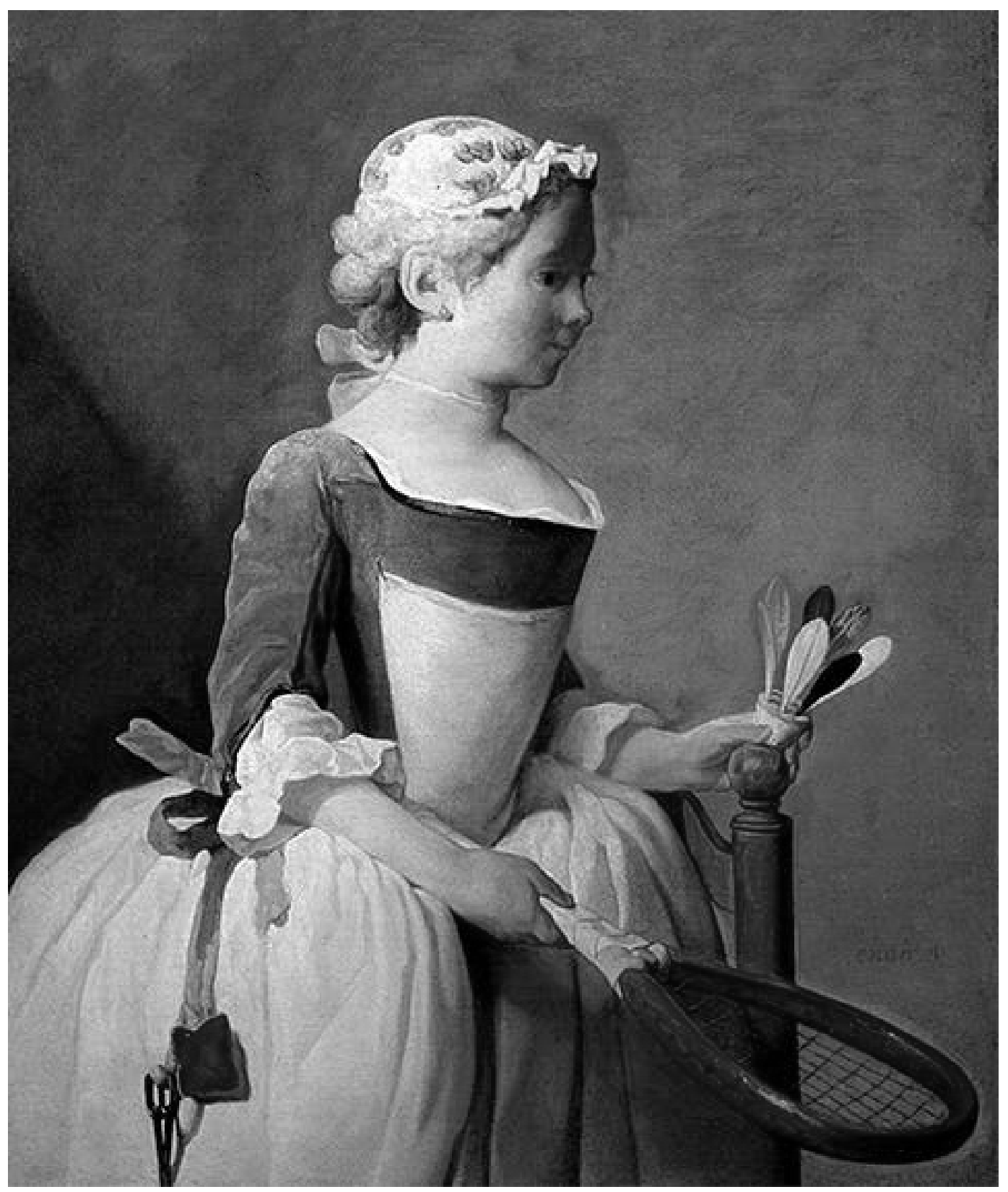

Figure 16 


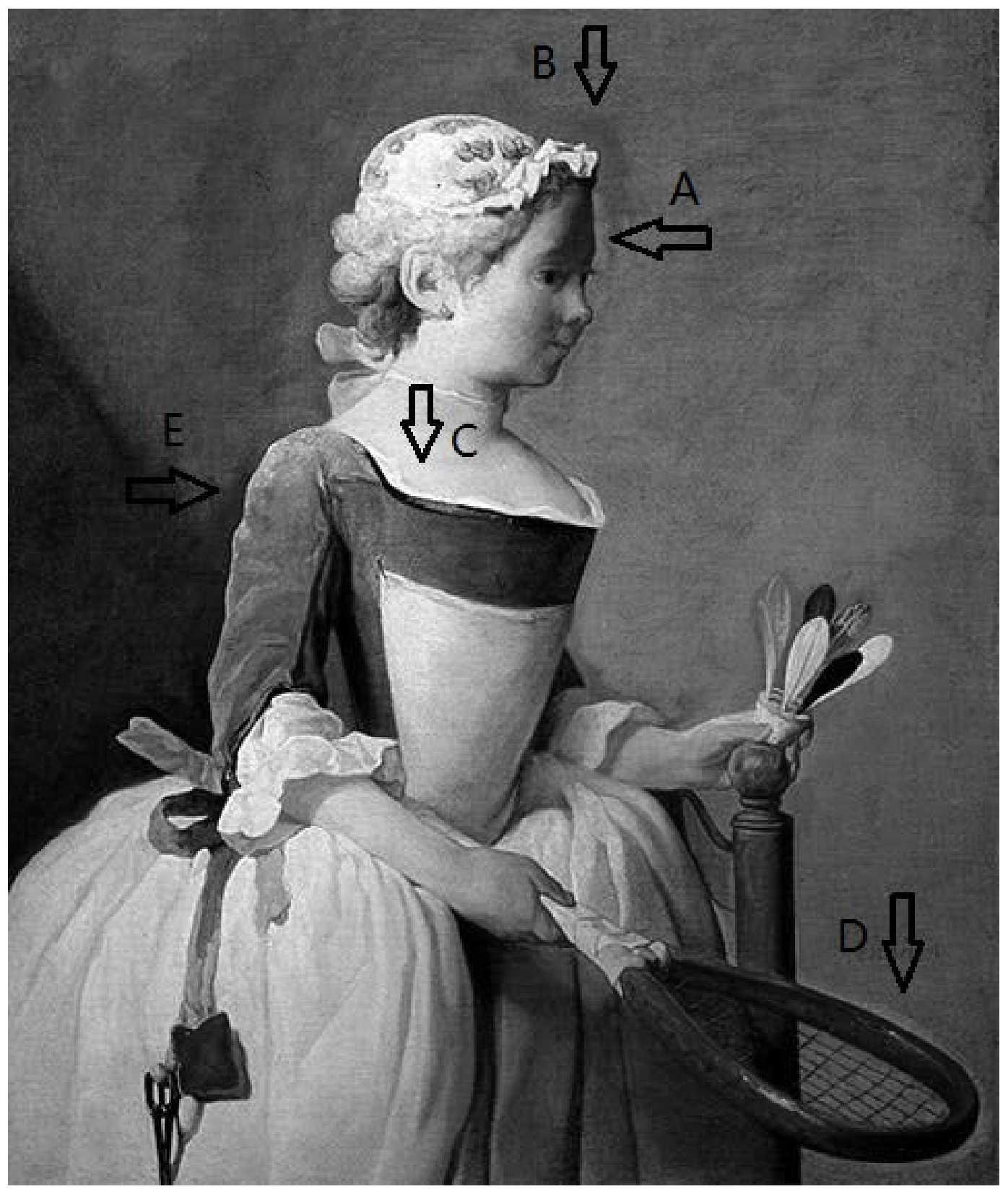

Figure 17

Here is one more example for this section. In Fig. 18, a monochrome reproduction of Study for 'Bathers at Asnières', by the Neo-Impressionist painter Georges Seurat, the contiguity illusion is evident around the two standing figures in the middle of the picture. In the original painting, with colour, the effect is even stronger. 


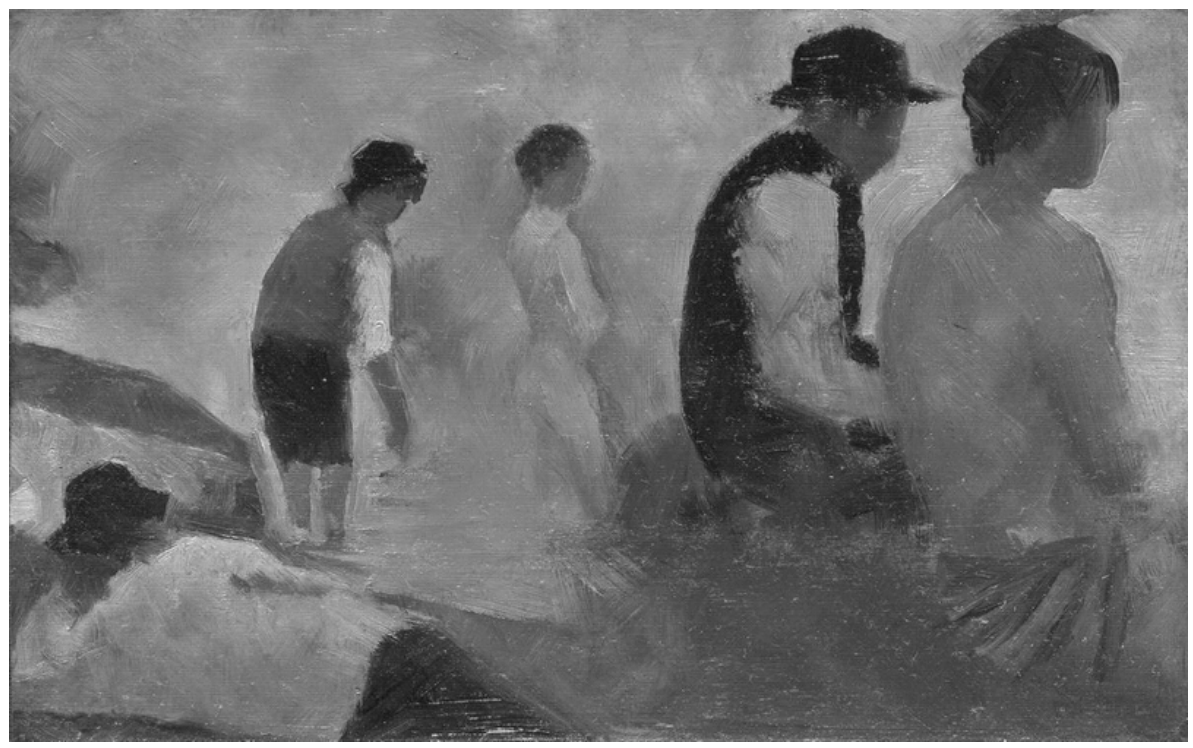

Figure 18

It is not difficult to find other Western masters in whose paintings there are noticeable depictions of the generalized Gibbs phenomenon: El Greco, Diego Velázquez, and Paul Cézanne, to name three more. Yet it is not, after all, only those masters in oil painting that do this; for instance, enhancing the bright and dark contrast around certain sharp boarders is a technique among the many things that the author was taught when learning drawing as a teenager.

\section{The checker shadow illusion}

In Fig. 19 a cylinder stands on a corner of what looks like a checkerboard, and casts on it a shadow, in which lies the square designated by the letter B, but not the one designated by $\mathrm{A}$. Although square B appears considerably brighter than square A to the human eye, the physical reality is that on this image the two of them have exactly the same brightness. Fig. 20 is the clean original image without the distraction of letters. Filtering this image with the filter shown in Fig. 21c, we get Fig. 21b, where at first sight the generalized Gibbs phenomena, though not the concern of this section, are easily noticed; Fig. 21a, the original image, is juxtaposed there for immediate comparison. Fig. 22a is the filtered image with two white horizontal straight lines going through square A and square B; the grey level values on the image along those two horizontal lines are plotted in Fig. 22b, with label A indicating those values from square A, and label B those from square B. As is clearly seen, the values of part A are far below those of $\mathrm{B}$, meaning that square $\mathrm{A}$ is evidently darker than square $\mathrm{B}$ after visual filtering, just as the checker illusion appears. 


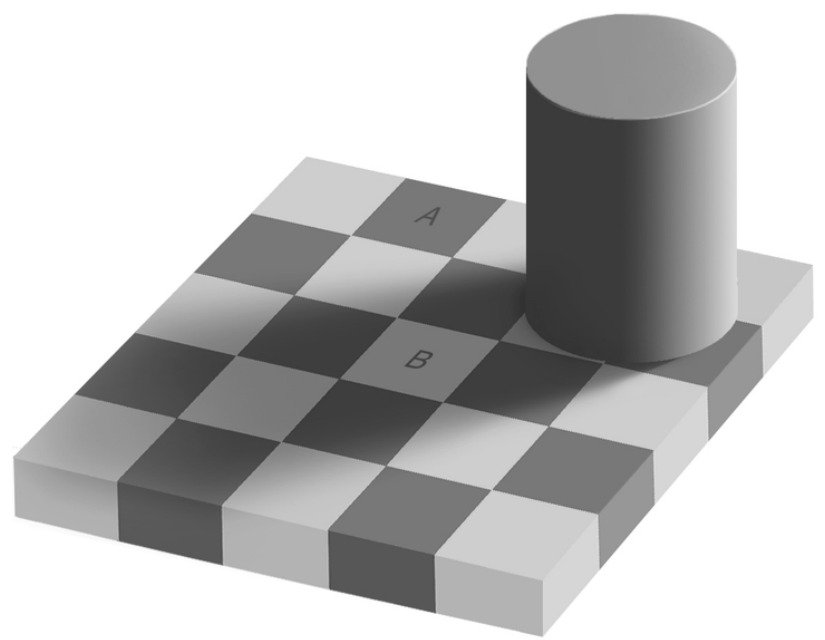

Figure 19

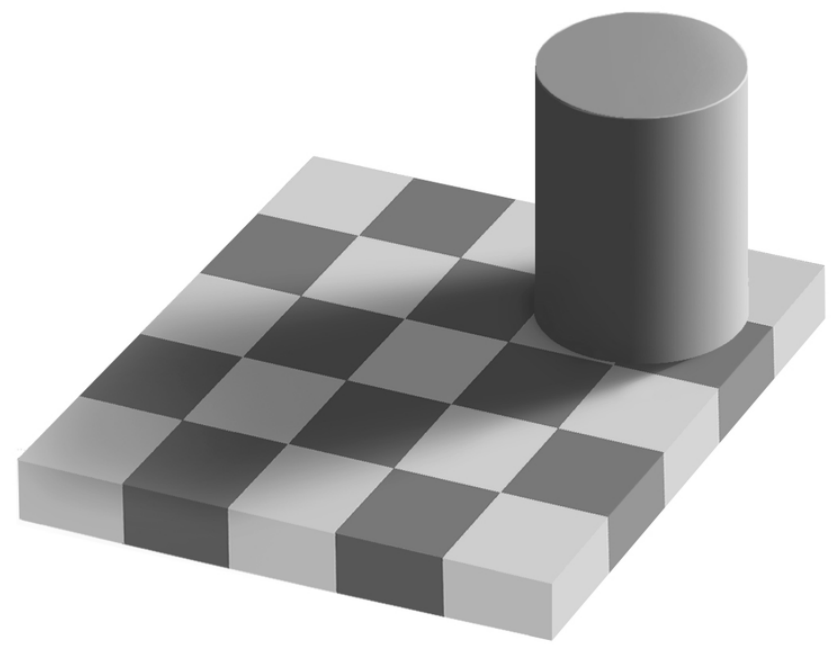

Figure 20

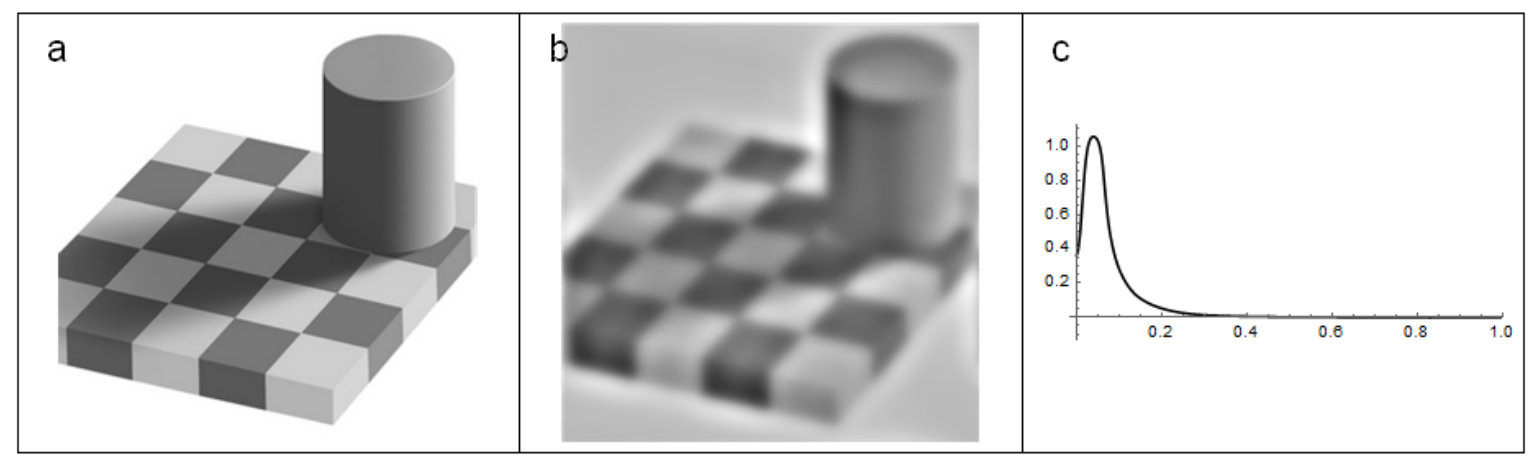

Figure 21 


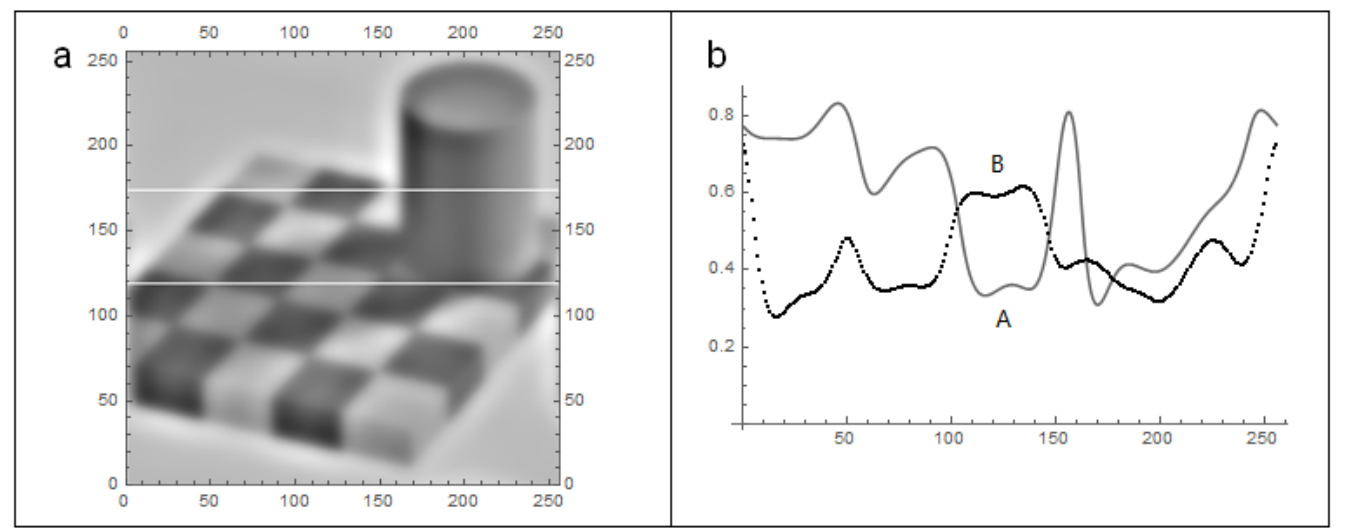

Figure 22

\section{The simultaneous contrast illusion}

In Fig. 23 the background is a colour gradient from black to light grey, and in the middle of the image is a thick horizontal bar. The bar appears to progress gradually from light grey on the left to dark grey on the right, but is, as a matter of fact, only one shade of grey everywhere within it. This visual illusion is very well explained by visual filtering based on Fourier analysis. Fig. 24 shows, from left to right, that original image, placed here for ease of comparison, the filtered image, and the filter used. Fig. 25a is the filtered image with a straight horizontal white line going through the central horizontal bar, and along that line the brightness values on the image are registered and plotted in Fig. 25b, where the part of the curve from $x=50$ to $x=200$ ( $x$ being the horizontal coordinate) is the values along that part of the horizontal line within the bar. Manifestly the curve shows that the left end of the 'filtered bar' is brightened by the filtering and the right end darkened, and that the brightness value on the bar gradually goes down from left to right. This matches the illusion.

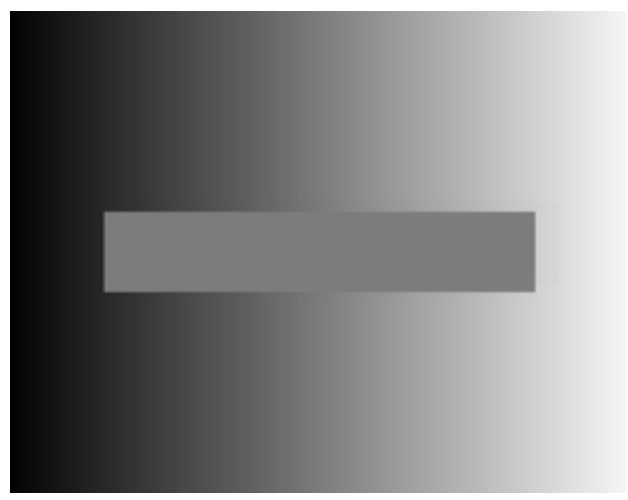

Figure 23 

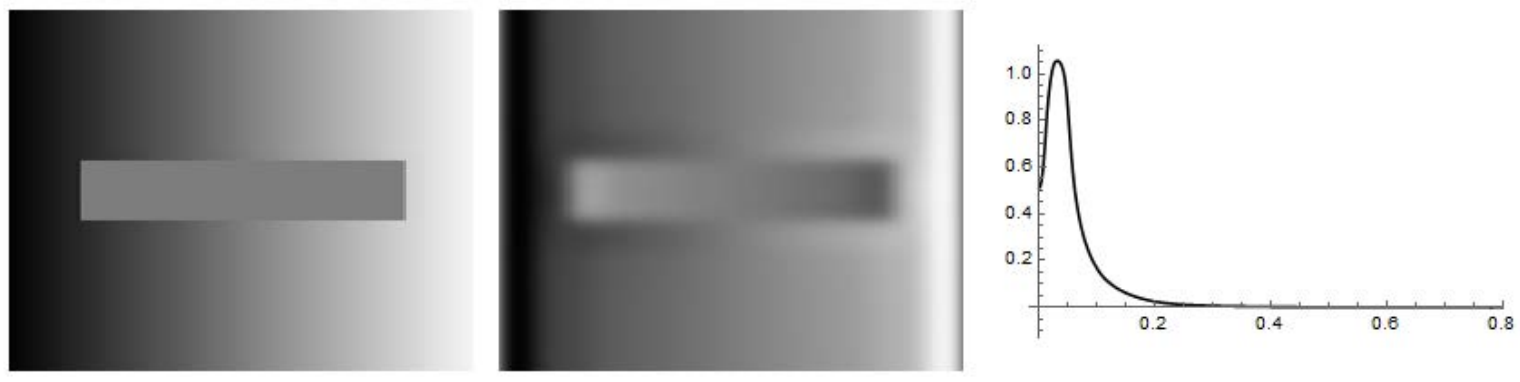

Figure 24

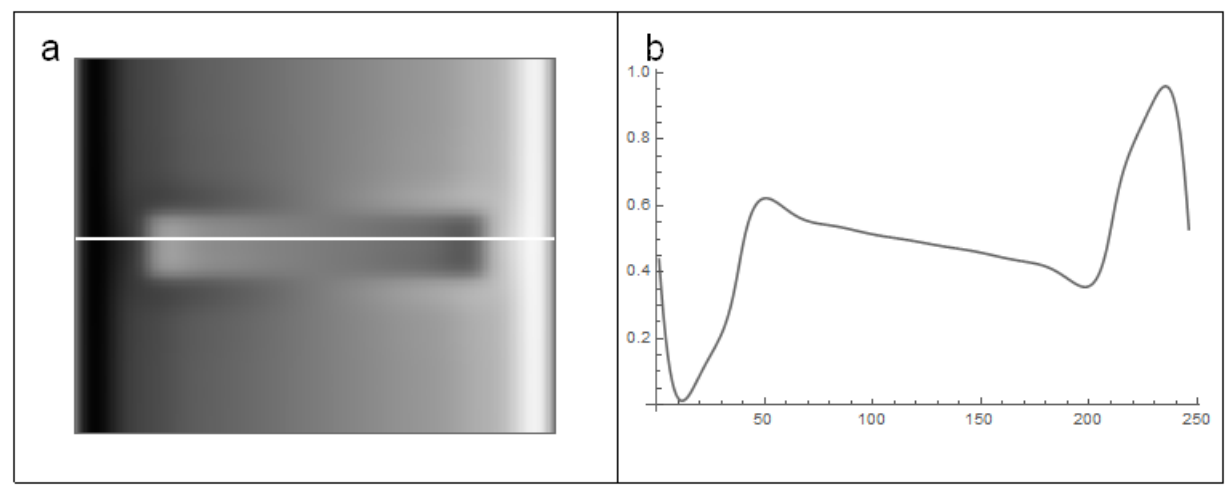

Figure 25

The darkening of the right side and the brightening of the left side of the horizontal bar as the result of visual filtering, although distorting the original image and accordingly regarded by scientists and engineers as sheer bad, unwanted artefacts, effectively enhance the balance, and the variety as well, possessed by the picture, and are therefore, in the eye of an artist (in the traditional sense), merits endowing the picture with a better composition and more beauty.

Another example of aesthetic improvement is that at the right edge of the filtered image there is a line darkened, and at the left edge a line brightened, both due to visual filtering, and together adding to the balance of the picture. There are also other changes worth noticing of this sort but they are not to be enumerated here. While the majority of people will find no merits in such effects, capable artists can see with their mind's eye the need for the existence of them, for aesthetic reasons mentioned just above. In other words, qualified and experienced artists are more sensitive than the layman to the kinds of visual illusions involved in their specialized artistic activity. Some of these visual illusions, however, may not be directly observable with the eye, but they can sense them with their mind's eye. 'Illusions seen with the mind's eye' may sound fictitious at first, but it is suggested here that they are real, because they follow the same principles as do the illusions perceived with the eye, and because, as was discussed previously and will be discussed further in some later sections, they are consistently depicted in and indispensable to many paintings by masters.

\section{Effects which are at once local and global}

From the checker shadow and simultaneous contrast illusions, it can be seen that Fourier visual filtering not only produces more enhanced light-dark contrasted contours via the 
generalized Gibbs phenomenon, but also changes the general light and dark pattern across the whole image. That second feature enhances the composition of the image in artistic terms, as was treated in the foregoing sections.

In good realistic paintings and drawings, correctly depicting large scale illusions of shades, such as the checker shadow and the simultaneous contrast illusions, is more important and more common, though far less noticeable, than the depiction of the continuity illusion (namely the generalized Gibbs phenomenon). As to what are good and what are bad realistic paintings, it is not a subject which can be dealt with in this paper, but well worthy of mention are that realistic paintings that look extremely similar to photographs are not aesthetically good, and that old masters of realistic (or, alternatively, naturalistic) painting, such as Diego Velázquez, did not paint photo-realistically at all (their works, though very realistic to the layman, by no means look like photographs to professionals in terms of colour as well as light and shade).

\section{Something obscure in the Hermann grid}

The commonly understood Hermann grid illusion (Fig. 26) is such that there are illusionary grey areas, or blobs, at the intersections of white passages; moreover, as a curious trait of it, those grey spots occurring at the intersections, though clearly visible, are destroyed when the white passages become wavy ${ }^{3}$ (Fig. 27).
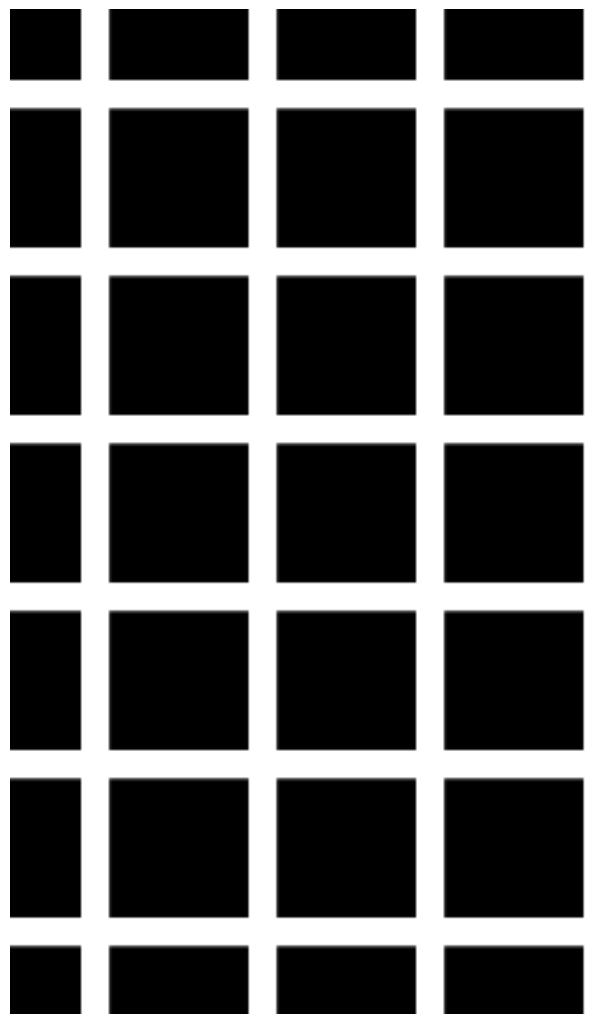
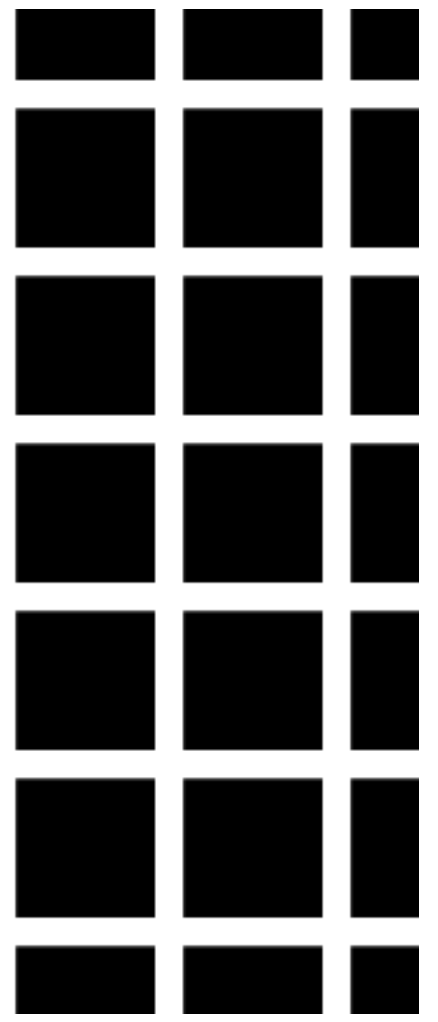


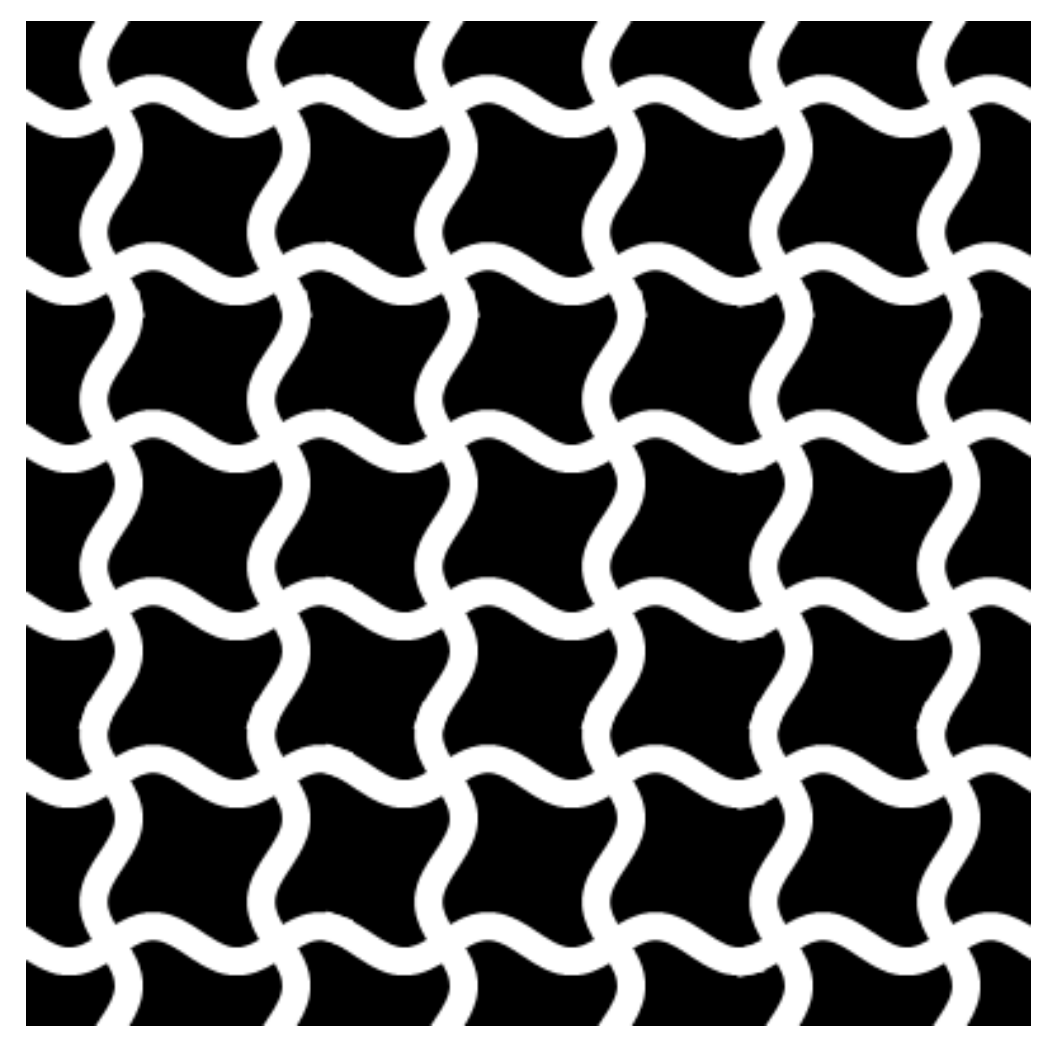

Figure 27

In this same grid pattern, there exits in fact another illusion, of a different kind, which is not at all as obvious: grey lines can be seen travelling along the middle of the white passages, and becoming thicker at the passage intersections, no matter whether the passages are straight or wavy (Fig. 30b and Fig. 31b). For better observation of it, probably the white passages should look wide enough to the eye (by enlarging the figure or letting it be close to the eyes), hence Fig. 28 and Fig. 29; also, it is advisable to gaze at the image for a relatively long time and with no movement whatever of the eyes.

That the Hermann grid illusion disappears when the white passages become wavy (Fig. 27) means that its generation is dependent on the rectilinearity of the grid pattern and is highly sensitive to deformation. Explanation of this trait actually involves key concepts from the study of geometric illusions with lines, to be presented later in this paper, and for that reason the common Hermann grid illusion can be called a vector brightness illusion. Those illusory grey lines described just now (Fig. 30b), by contrast, do not disappear when the white passages become wavy (Fig. 29 and Fig. 31b). So this second illusion is not sensitive to deformation, and can therefore be called a raster brightness illusion (for being independent of the rectilinear shapes involved). Visual filtering as described above can account this phenomenon well. 


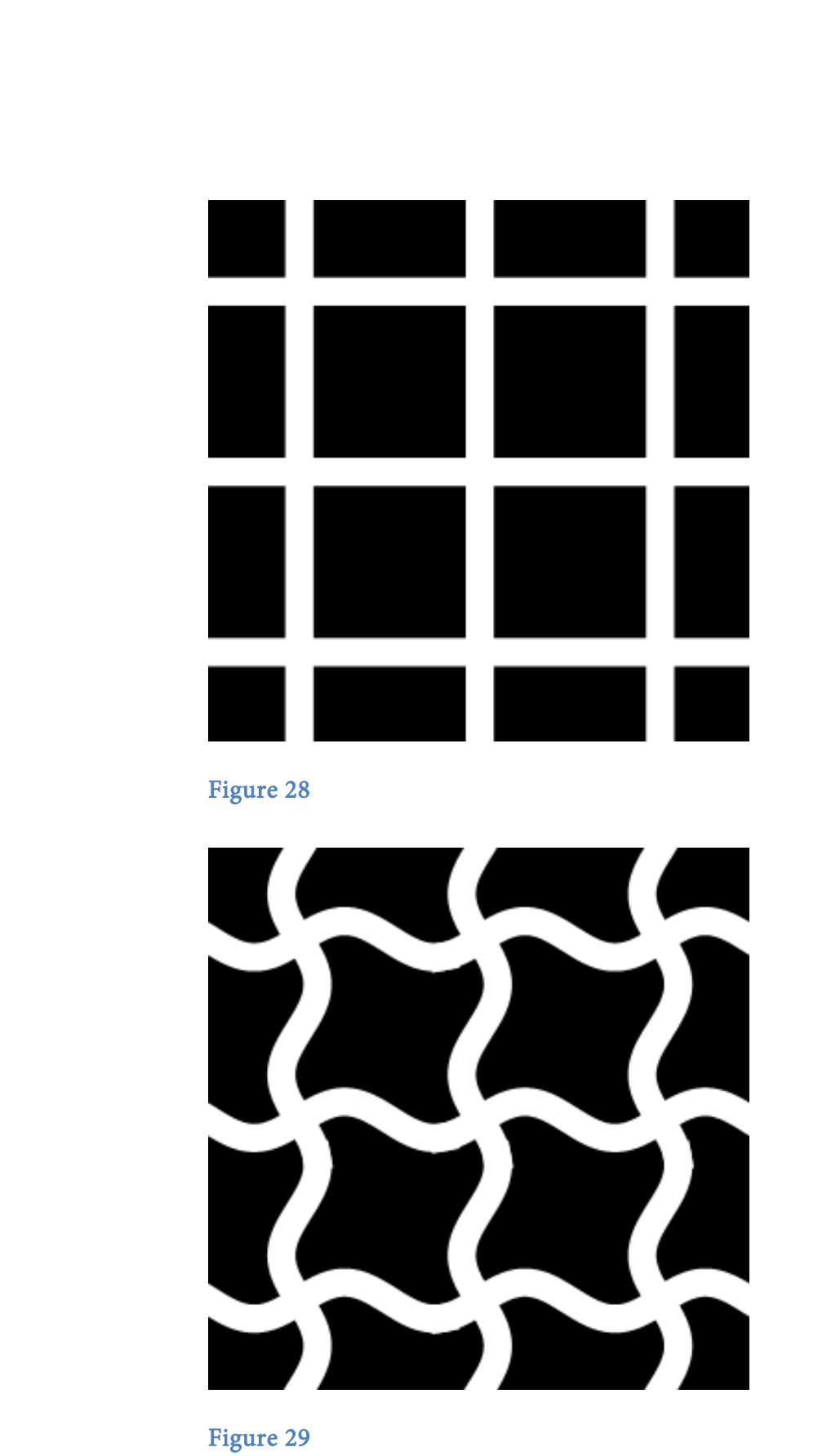


Fig. 30a is an image of the Herman grid used for visual filtering, where how many cycles of black squares there are horizontally or vertically does not really matter since in visual filtering the image in question is always taken to be periodical. Shown in Fig. 30c is the filter used, and the result is given in Fig. 30b. The same goes for what are presented in Fig. 31, which shows that this raster illusion is not conditional on the rectilinear shapes of the white passages, and remains undiminished by that shape distortion. The adoption of the word raster is also due to the fact that the form of visual filtering which has been used in Fig. 30 and Fig. 31, and in all previous brightness illusions, processes the image - or rather, any image - in a way similar to raster-scanning, not caring in the slightest about what shapes there are in the image. Vector brightness illusions, on the other hand, do not accept such filtering.

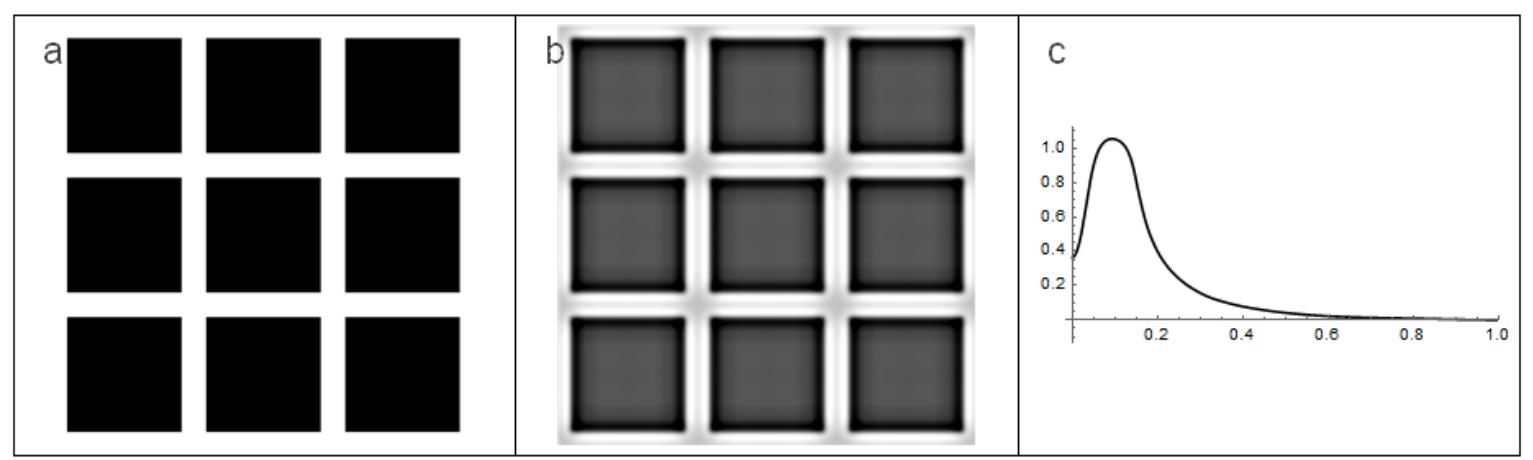

Figure 30

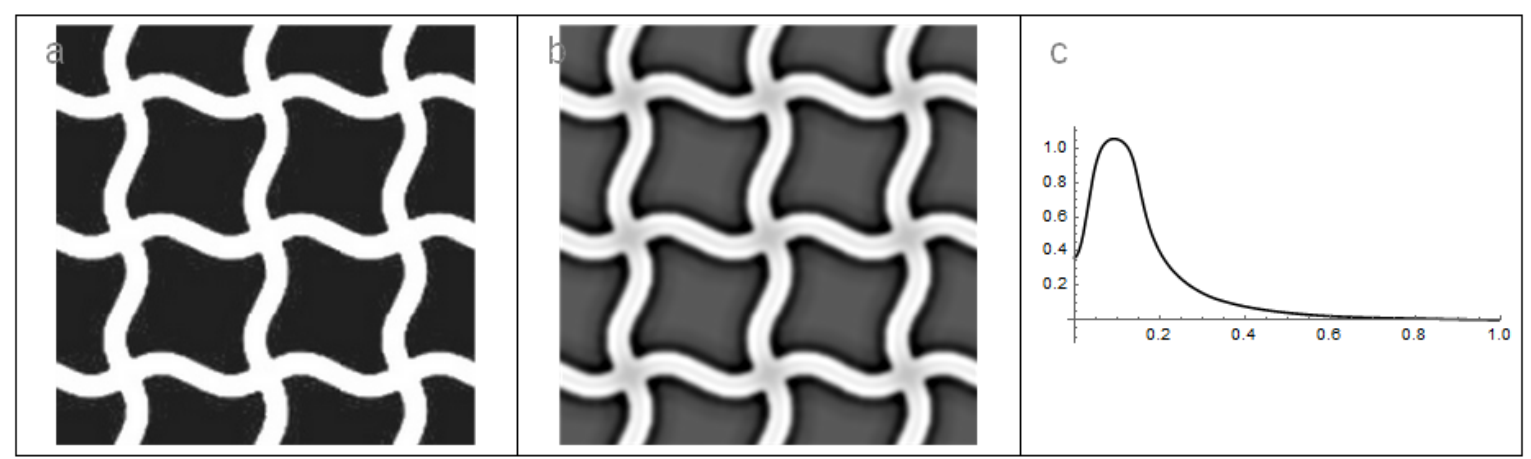

Figure 31

In addition, it is well known that there has been the attempt to explain the Hermann grid illusion through the lateral inhibition theory, which attempt fails however to account for the disappearance of the illusion when the white passages are wavy. What the lateral inhibition theory explains in this case is actually right this raster illusion hidden in the Hermann grid: with a properly chosen convolution mask (namely the lateral inhibition function as in Fig. 7), the grey lines in the middle of the white passages (as in Fig. 30b and Fig. 31b) can also be generated by convolution of the image in the space domain, a process equivalent to filtering in the frequency domain.

An explanation of the common Hermann grid illusion itself and the scintillating grid illusion as well, both of them vector brightness illusions, will be given in a section far below, after discussions of some geometrical illusions. 


\section{Colour illusions}

When it comes to the simulation of colour perception of human vision, one has to deal with the problem of what colour system (or colour model, with the same meaning) to use, there being various models such as the RGB, CMYK, Munsell, LAB, HSV, and HSL models. The best choice is the Munsell model, because, invented by an artist, it matches the human sensations of colours the best. For example, in this model, colours of the same value (namely, the number indicating brightness) indeed appear to have the same brightness - that is, to be as bright as each other - to the human eye, whereas in the LAB, HSV, or HSL model, colours of the same 'brightness', 'value', or 'lightness', respectively, are clearly not of the same brightness to the human eye. Moreover, complementary colours given by the LAB, HSV, and HSL models may not be completely as felt by the human visual system.

Colour image processing in the present paper uses a self-made colour model that mimics the Munsell model; in more detail, it can be thought of as a modified HSV model such that all colours of the same value, no matter what this value is, appear to the human eye to be of the same brightness (i.e. as bright as each other) - unlike the standard HSV model. (Utilising the HSV or HSL model, or even the RGB model, can generate results roughly similar to those presented below but of much less satisfactory quality.) The following words in this section give some technical details of colour Fourier visual filtering, which can be skipped by a reader that does not plan to replicate the experiments.

Suppose that we now have a suitable colour model. Whether or not it is the real Munsell model that is used, the model's colour space, of a highly irregular three-dimensional shape, has to be stretched or squeezed in the saturation axis, namely for each $(h, v)$ pair, in order for the colour space to have the shape of a cylinder, otherwise the model is unsuitable for visual filtering.

When that colour model is ready, it is still not immediately suitable for use in the colour image filtering process, because it specifies points in the colour space with a cylindrical coordinate system (in which $v$, the 'value' of a colour, ranging from 0 to 1 , is the $z$-axis). Holding $v$ constant, we have an $h$-s (hue and saturation, both ranging from 0 to 1 , too) plane of constant brightness, on which each point is specified by this pair of polar coordinates: $h$ $(0 \leq h \leq 1)$, a number in proportion to the direction angle (ranging from $0^{\circ}$ to $360^{\circ}$ ) of the point, and $s(0 \leq s \leq 1)$, the distance of the point from the axes centre. What needs to be carried out is to convert this polar coordinate system of $h$ and $s$ into a Cartesian coordinate system, which is easy. After the conversion, a colour $(h, s, v)$ now becomes the $(x, y, v)$ of a threedimensional Cartesian coordinate system for colours. It is in this system of $(x, y, v)$ that the Fourier visual filtering of colour images is performed, which involves independent onedimensional Fourier transforms (and filtering) on the $x, y$, and $v$ directions.

In addition, visual filtering based on this $(x, y, v)$ system corresponds to some major techniques of oil painting. More specifically, filtering in the $v$ direction contributes to the drawing aspect of the painting process, which aspect pays attention not to the colours in the scene, but to the light and shade in the scene being painted and the solid forms of the objects therein, and the artistic representation thereof; filtering in the $(x, y)$ plane, on the other hand, contributes to the colour aspect of the painting process, which is responsible for the observation of the complex colour relationships within the scene, and for the proper artistic 
representation of them in the painting, especially the rhythmical use of complementary colours.

\section{Examples of colour visual filtering}

In the first panel of the three in Fig. 32, a monochrome horizontal bar of which every part is of the same grey, completely colourless, is placed in front of a background whose colour changes gradually from red on the left to cyan on the right. Despite its complete lack of colour, the left side of the bar appear cyan-ish to the human visual system and the right side reddish; this is in reality a colour version of the monochrome simultaneous contrast illusion (Fig. 23). It has been simulated by colour visual filtering, which uses a special colour model as described in the previous section, and the filter in the third panel of Fig. 32; the result is given in the second panel of Fig. 32. To make sure that the result is indeed representing the illusion experienced by the human visual system, two points are chosen (as the respective centres of two little circles) at the left and the right end of the horizontal bar in the filtered image (Fig. 33a), and then the colours at these two points are presented as two swatches, placed in the same order, in Fig. 33b. The left swatch is cyan-ish and the right one reddish, each clearly matching our illusion.

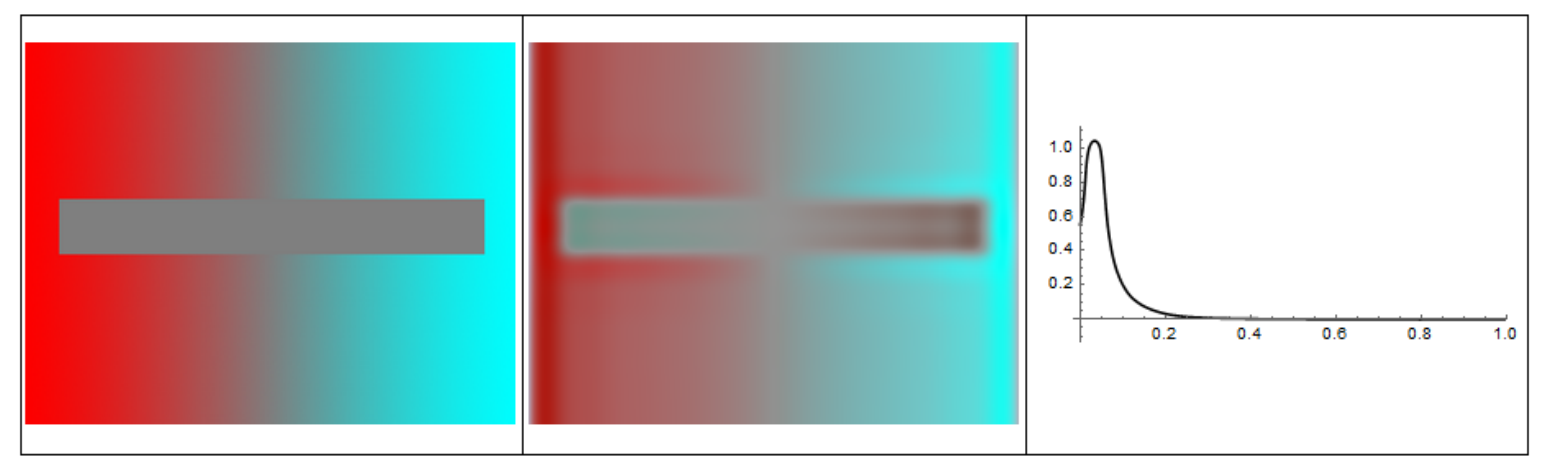

Figure 32

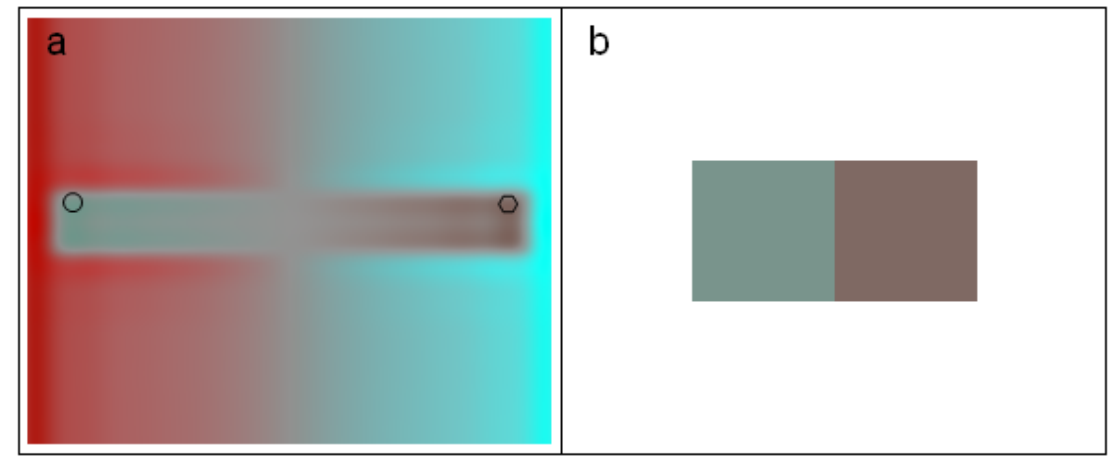

Figure 33

In Fig. 34, placed side by side are two images of a cube which are identical except that on the right one two labels, $A$ and $B$, have been added so as to identify two little squares on the cube. Square A and square B are really both of the very same brown colour, but their colours appear drastically different to the eye, with that of A being yellow and much brighter than 
that of B. This illusion is reproduced well by means of colour visual filtering (Fig. 35b, with Fig. 35c showing the filter used). Of the filtered image, the colour at the centre of square A and that at the centre of B are juxtaposed, in the same order, in Fig. 36b, clearly showing their distinction, as it is felt in the illusion. Admittedly the swatch to the right looks a bit too dark and also that to the left possibly a bit too bright, a sign that the filtering was a little overdone, due mainly to the fact that some low frequency components of the image, especially some of the lowest, were over-reduced.
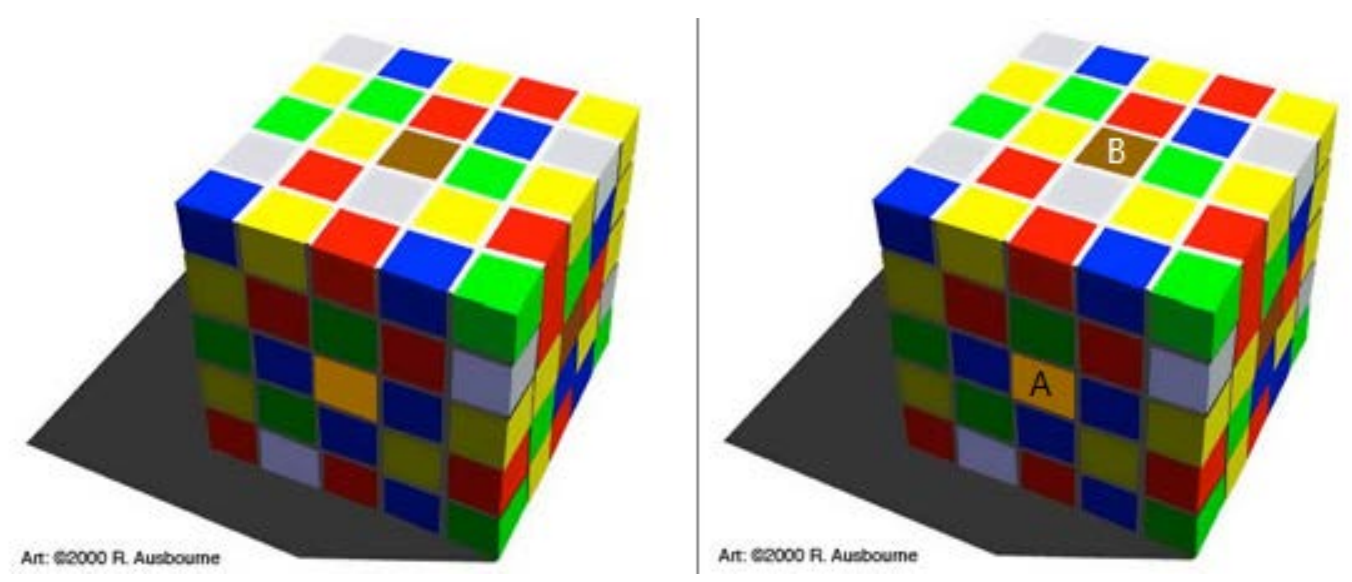

Figure 34

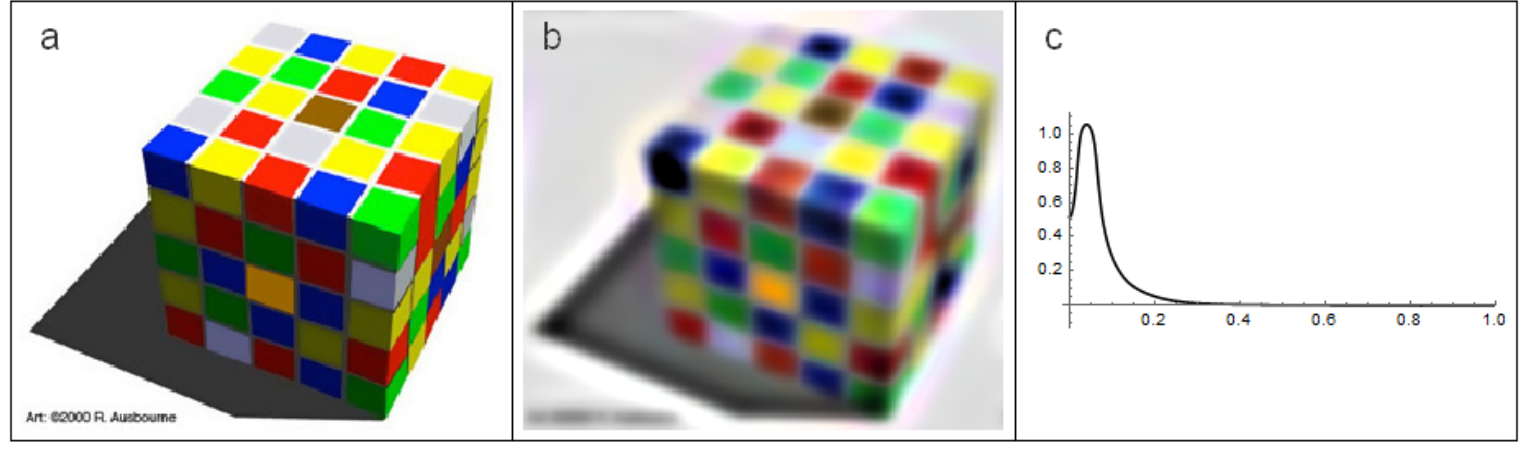

Figure 35

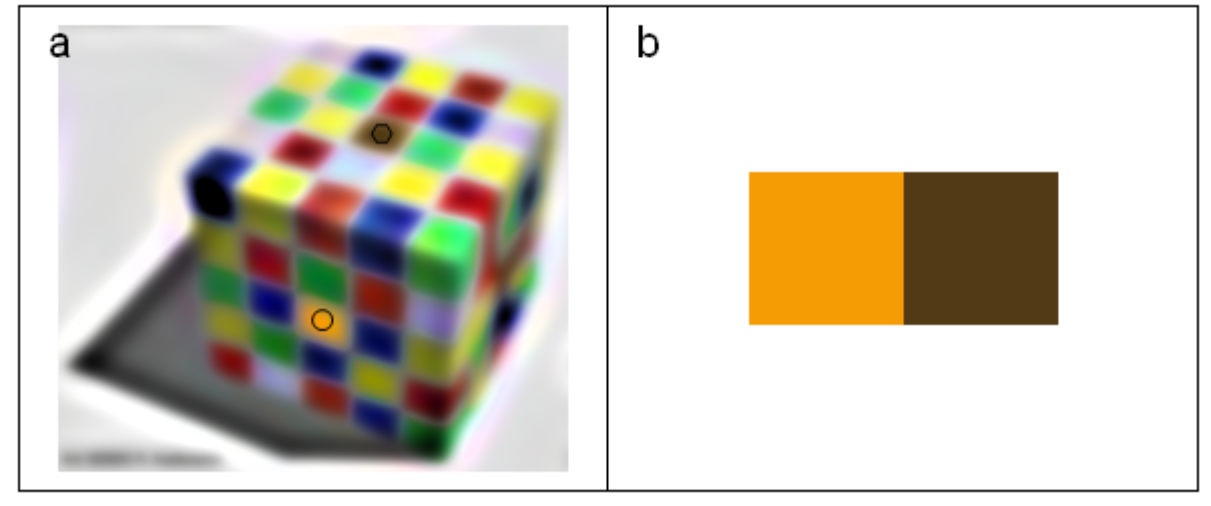

Figure 36

As with brightness illusions and visual filtering of monochrome images, colour illusions and visual filtering of colour images too are able to increase aesthetic aspects of the images in 
question. Further discussion of it will be found in the section Aesthetic elevation of the colours of an image, near the end of this paper.

\section{Geometric illusions with lines}

In China, 书法 ( $\mathrm{Shu} F a$ ), translated into English, in fact with great loss in significance, as calligraphy, is a traditional, vast, and deep form of art, with a very long history and many great old masters. This art seems to be closely relevant here: geometric illusions are created by special arrangements of lines (not necessarily straight) or of geometrical shapes, whose contours are still lines; Chinese calligraphy is, in an important sense, an art of lines.

In historical discussions of Chinese calligraphy, there is one sentence, describing the process of appreciation of works by masters, that is most likely to reveal a truth about human visual perception of lines: ‘余尝历观古之名书, 无不点画振动, 如见其挥运之时。’. Quoted from 《续书谱》, a discourse on calligraphy written in the 12th century by the calligrapher and poet 姜醬 (Jiang Kui), those words can be translated as this: When I admire old masterpieces of calligraphy, to me the strokes in them always appear flowing, as if I were directly watching their authors moving their writing brush above the paper and creating those brush strokes. The remark means that when appreciating a masterpiece of calligraphy, he mentally reconstructs the process of its author creating that particular work, simulating in the mind the motion of the author's brush on that paper. Hence it was virtually pointed out by Jiang Kui that the calligrapher, when looking at calligraphic lines, does not process these lines in the mind in a static manner, but dynamically instead; in other words, the calligrapher's mind 'sees' the lines as motion.

What the calligrapher's mind sees is another example of one thing noted earlier, namely that qualified and experienced artists are more sensitive than the layman to the kinds of visual illusions involved in their specialized artistic activity. Nonetheless, the recognition that lines tend to imply motion is not limited to artists only: people in general when so told and looking attentively at appropriated pictures (such as Fig. 37a) are able to sense it too (like the flowing movement of the curve indicated by arrows in Fig. 37b). Thus we can hypothesize with good reason that lines are intrinsically motions to human vision. 


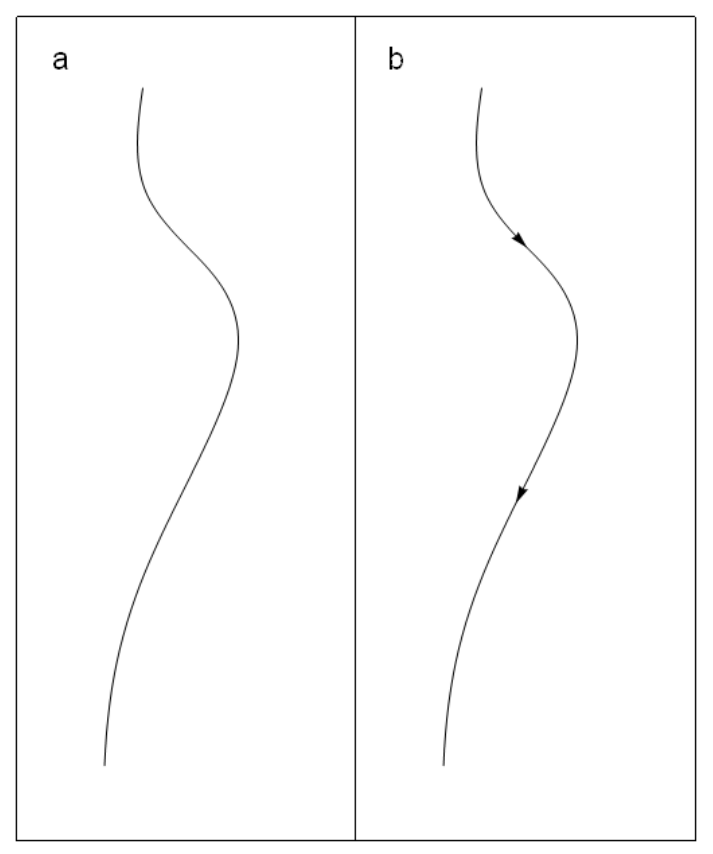

Figure 37

Now we interpret the lines in geometrical figures as trajectories of moving particles (or, more vividly, dots), treating each separate line as one virtual moving particle for the sake of simplicity, and subject their movements to visual filtering with Fourier analysis.

One question immediately arises: a line, either straight or curved, is a static thing; what speed values do we assign to points on it so that the line can become a trajectory we need? The simplest approach is to let every point on it have the same constant speed, and see whether the result of visual filtering is satisfactory enough, which is what will be adopted in the following discussions.

The line figures investigated in this paper are of two types, the simple single-path figure and the vertex multi-path figure. A simple single-path figure is here defined as a figure consisting of one continuous line that does not intersect itself or contain acute angles, so that there is only one virtual moving particle involved, which creates one single path (e.g. each of the three lines in Fig. 38a). A vertex multi-path figure is here defined as one wherein multiple lines meet at a common point, the vertex, and the situation involves multiple virtual particles moving simultaneously, each creating its own path through the vertex, and interacting with each other in the process of producing visual illusions (Fig. 38b). 
a

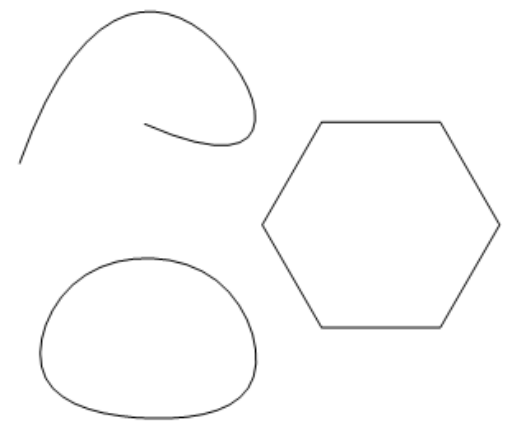

$\mathrm{b}$

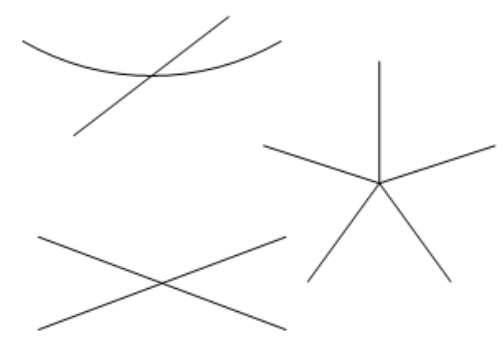

Figure 38

An acute angle, alone, its two sides meeting at the vertex, is in fact primarily a vertex multipath figure. That can be decided from and illustrated by the case of a very sharp angle, as in Fig. 39a, where the two angles drawn are the same, with one of them labelled. It appears clear, though difficult to prove, that, if we look at that angle, the perception process, or at least our attention, the subconscious part of it perhaps, tends to start from the vertex $\mathrm{O}$ and go down the two sides OA and OB separately but simultaneously, and finally (when OA and OB are of the same length) to reach points A and B simultaneously too. Going in the opposite direction is also highly possible, with $\mathrm{A}$ and $\mathrm{B}$ as the simultaneous starting points and $\mathrm{O}$ as the common end point. Thus $\mathrm{AO}$ and $\mathrm{BO}$ are the two paths existing for virtual moving particles in this angle.

If $<\mathrm{AOB}$ is a large obtuse angle (Fig. 39b), for instance of 172 degrees, it seems evident that the path is either $\mathrm{AOB}$ or, equivalently, its reverse, $\mathrm{BOA}$, that the simultaneous paths of $\mathrm{AO}$ and $\mathrm{BO}$ are impossible because $\mathrm{A}$ and $\mathrm{B}$ are too far away from each other, and therefore that the angle $<A O B$ is a single path figure.

Such words about Fig. 39 are, of course, some assumptions, whose validity or invalidity depends on whether or not they lead to models that match the corresponding illusions. Thus, from an extremely small acute angle (e.g. of 1 degree) to a nearly 180-degree obtuse one (e.g. of 179 degrees), there ought to be a gradual transition from a complete multi-path figure to a complete single-path figure, with the angles in between being a varying mixture of both. In other words, the percentage of single-path-ness of an angle (for simplicity of description, the sizes of angles involved here are always between 0 degree and 180 degrees) is a monotonously increasing function of the size of the angle. 


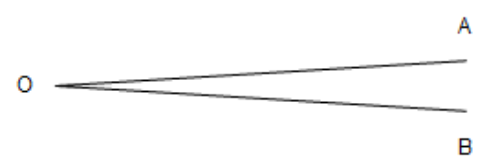

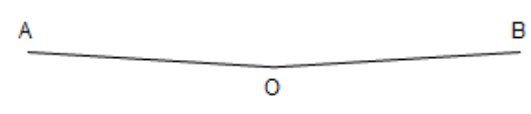

Figure 39

A simple-path figure as defined just now may also be multi-path in some other circumstances; for instance, in both the smooth closed curve in Fig. 38a and the smooth wavy curve in Fig. 64, it seems also possible that the visual processing of the curve starts from the central, highest point of the curve, i.e. the part of the figure that first catches the viewer's subconscious attention, and goes down the left and the right sides of it simultaneously, in a more or less symmetric manner. Further investigation into this matter goes beyond the concerns of this paper.

\section{Choice of kinematic quantities}

Yet another question arises too: in view of the fact that, at any instant, to describe the state of the particle's motion, there are such quantities as position, velocity, and acceleration, which of them should be used to represent the movement of the particle and then undergo visual filtering? (The terms speed and velocity in this paper are used exactly as they are in physics, where speed is the magnitude of velocity, which, as a vector quantity, has direction as well.)

For simple single-path figures, this problem can be satisfactorily solved, because, as a matter of fact, it does not exist: the mathematical properties of Fourier analysis determine that subjecting any of the three quantities to visual filtering by the same filter will finally give the same resultant trajectory, and for that reason the three of them do not make any difference as to visual filtering. Naturally the simplest of them, the particle's position as a function of time, i.e., trajectory, will be used when dealing with single-path figures.

There is still another quantity that is likely to be quite a qualified candidate for the purpose of visual filtering: path curvature, that is to say the mathematical concept of curvature at any point of the path curve. This concept, a purely geometrical one, is inherently different from all the other three (position, speed, and acceleration) in that if used it has the effect of treating the motion of the virtual moving particle as always maintaining a constant speed. The other three quantities, by contrast, are kinematic concepts, and allow speed variation along the path, and are therefore dynamic; they are strictly necessary when real motion illusions of moving particles are studied. Moreover, curvature is mathematically more complicated than velocity and acceleration. (If, in addition, there are corners along a path, the curvature at a corner is, strictly speaking, infinite; fortunately, that infinity can be eliminated by discretisation of the path.) Hence curvature is not used for visual filtering in this paper, but it may be a good enough concept to work with in many cases. 
For vertex multi-path figures, however, things become much more complicated and the above analysis for single-path figures no longer applies when dealing with interactions between different lines. The details will be seen in the corresponding part below.

\section{Simple single-path figures}

Such figures, of which the definition has been given, are the simplest cases in the discussion of geometric illusions with lines, because here there is no interaction between different lines - hence no interaction between different virtual moving particles, and because, as has been explained, the positions of the particle can be used directly for filtering.

Application of visual filtering to a line requires first the determination of the motion of the imaginary moving particle along the line. A constant speed motion, the simplest of all, generally suffices for a meaningful result. The particle's motion having been decided, parametric equations of it should be given, namely, its position $(x, y)$ as a function of time (all lines here considered are two dimensional, and the Cartesian coordinate system is used):

$x=x(t) ; y=y(t)$.

Next comes the question of what happens when the virtual particle reaches an end of the line if it is not a closed curve. In this paper, it is supposed that the virtual particle bounces back, so to speak, at each end of the line, and then follows the line in the opposite direction, so that its path still forms a closed loop (Fig. 40).

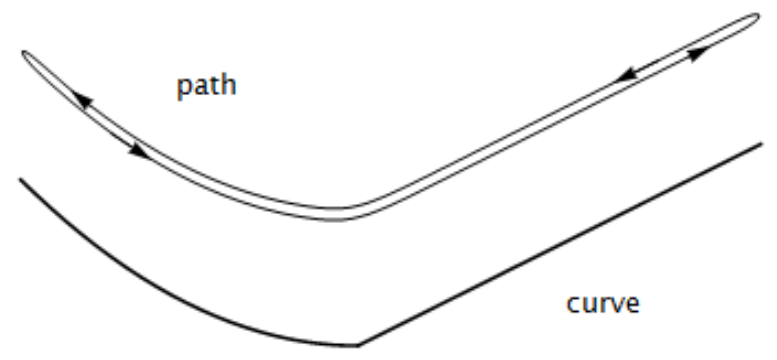

Figure 40

Afterwards, the parametric trajectory is discretized into a large number of sample points, with equal and very short time intervals between consecutive ones. These sample points then undergo visual filtering, which, more specifically, consists of two independent filterings on the $\mathrm{x}$-direction data and the $\mathrm{y}$-direction data.

\section{The bulb illusion}

The curve for producing this illusion consists of an arc and two straight line segments that are joined to and tangent to the arc at the two ends of it (Fig. 41a, which is the leftmost panel of the four in Fig. 41, the label 'a' having been omitted so as not to pose distraction to 
observation of the shape). Because the line segments are tangent to the arc at the joints, the arc should not appear to be a swelling bulb with respect to the two lines; but to the human viewer it does appear that there are little swellings at the ends of the arc, such that the perceived appearance of the arc resembles, to a certain degree, the shape of a swelling light bulb. Filtering the path of this shape with a filter as shown in Fig. 41d, we get the resultant shape in Fig. 41b, which distinctly delineates the illusion. Fig. 41c shows the filtered curve, in black, placed on top of the original curve, now in grey. The same is true of Fig. 42, where the arc, in this case, is a much larger portion of a circle.

This illusion is also an example of the contiguity illusions mentioned earlier, a manifestation of the generalized Gibbs phenomenon, since at either joint we have a circular motion on one side and a straight line motion on the other, namely a discontinuous jump of the moving particle's path curvature as well as acceleration. (In the configuration in Fig. 42, there is an intersection of the two straight lines, thus creating a multi-path vertex there, but this is not the concern of the current bulb illusion problem and is therefore ignored here.)

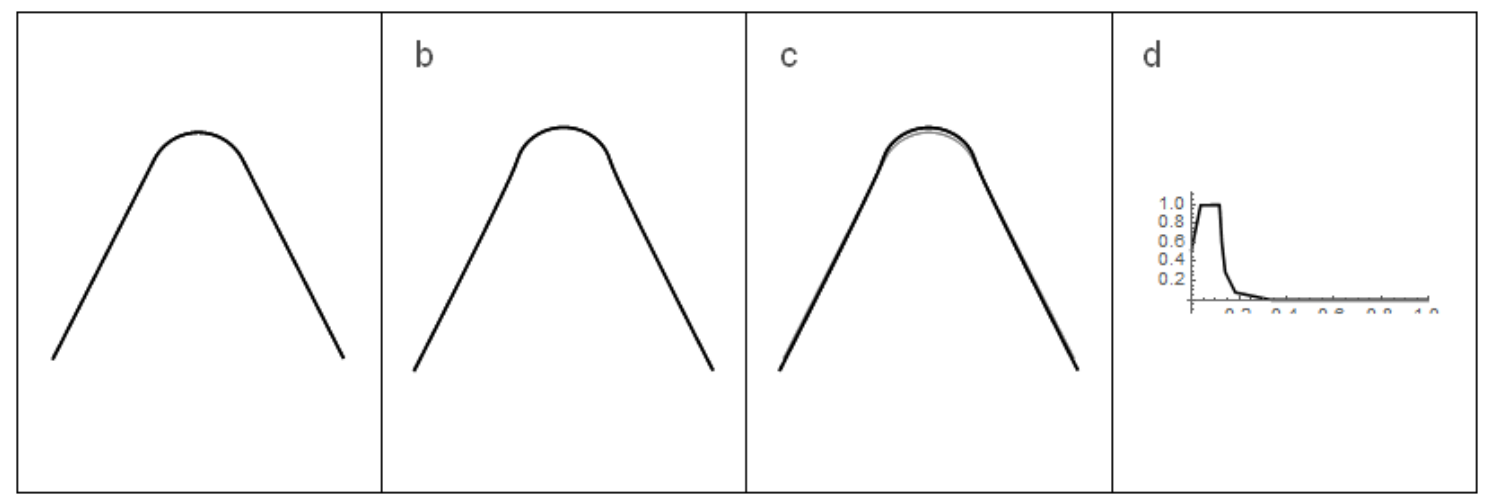

Figure 41

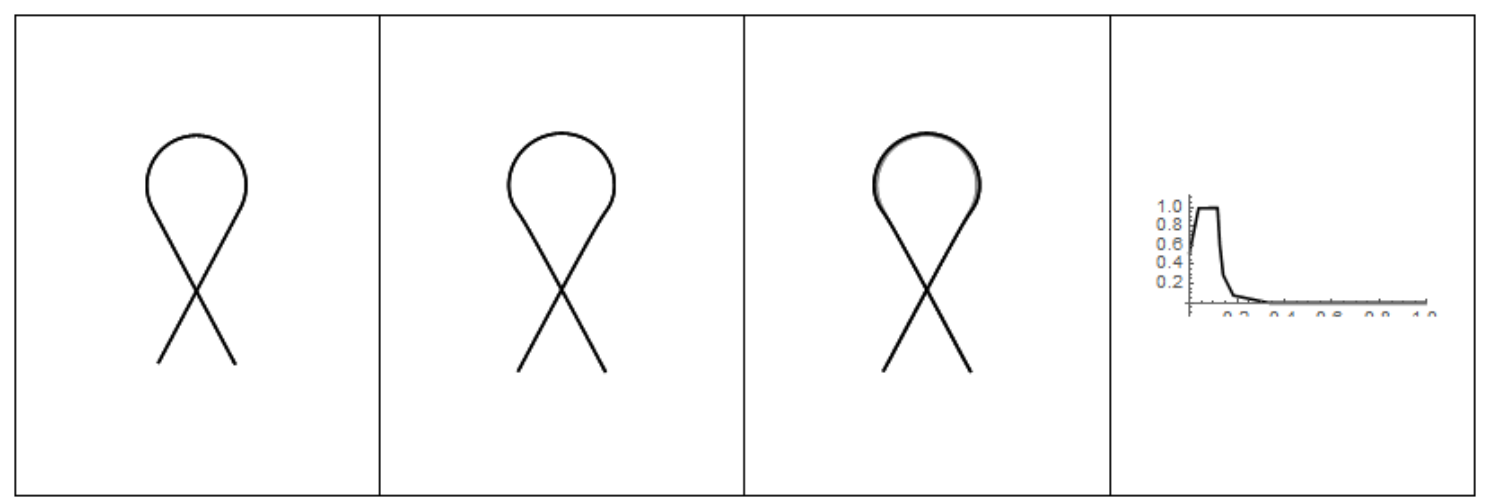

Figure 42

\section{Deformation of single-path angles}

Unusual subtlety dwells in this matter. When acting as a single-path figure, an angle, whether acute, right, or obtuse, will appear very slightly smaller to the human observer than it really is; furthermore the sides of it will not look perfectly straight. An alternative description is that the angle appears a bit sharper than it really is when near its vertex. For instance, treating the 
acute angle in Fig. 43a (the left panel in Fig. 43, with label 'a' omitted) as a single closed path (as in Fig. 40), and subjecting it to visual filtering with the filter in Fig. 43c, we get the sharpened angle in Fig. 43b; the same goes for Fig. 44. As illustrated with more clarity in Fig. 45, where the results of Fourier visual filtering (in red) are laid on the original angles (in grey), the illusionary angle is always smaller than the original regardless of whether the latter is acute or obtuse.

Again, this is an example of the contiguity illusion, a manifestation of the generalized Gibbs phenomenon, due to the abrupt change in the direction of movement at the vertex.

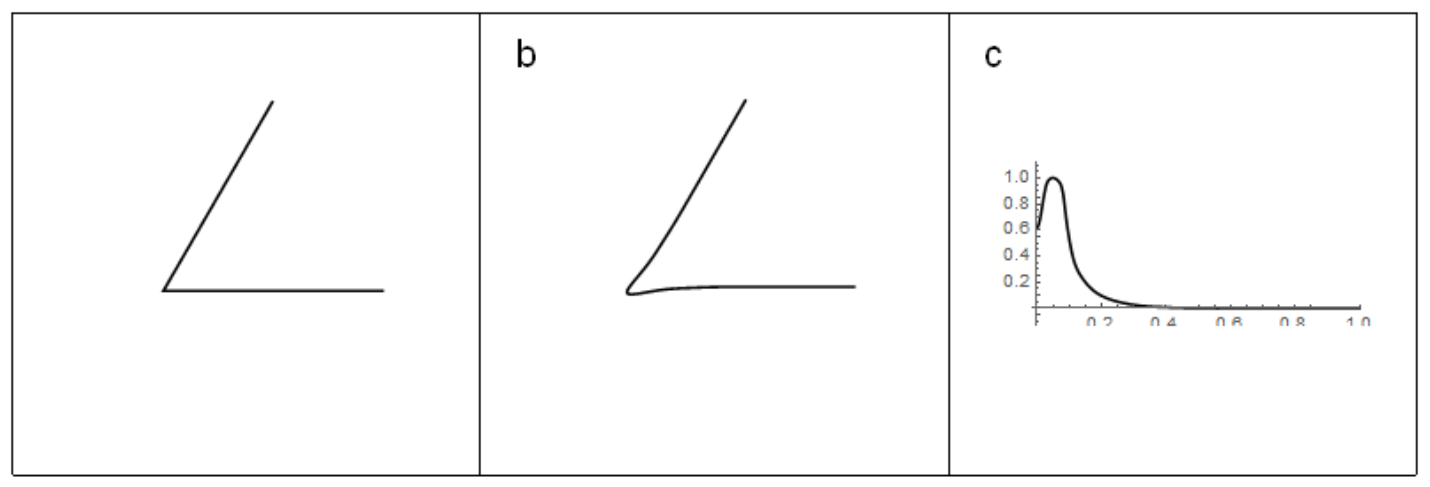

Figure 43

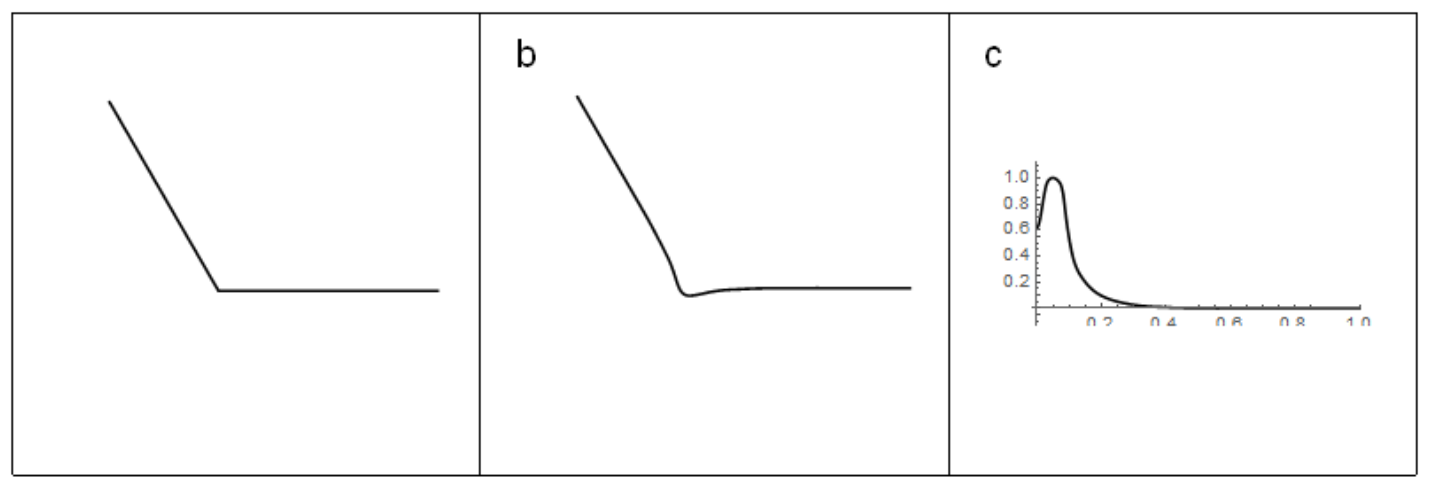

Figure 44

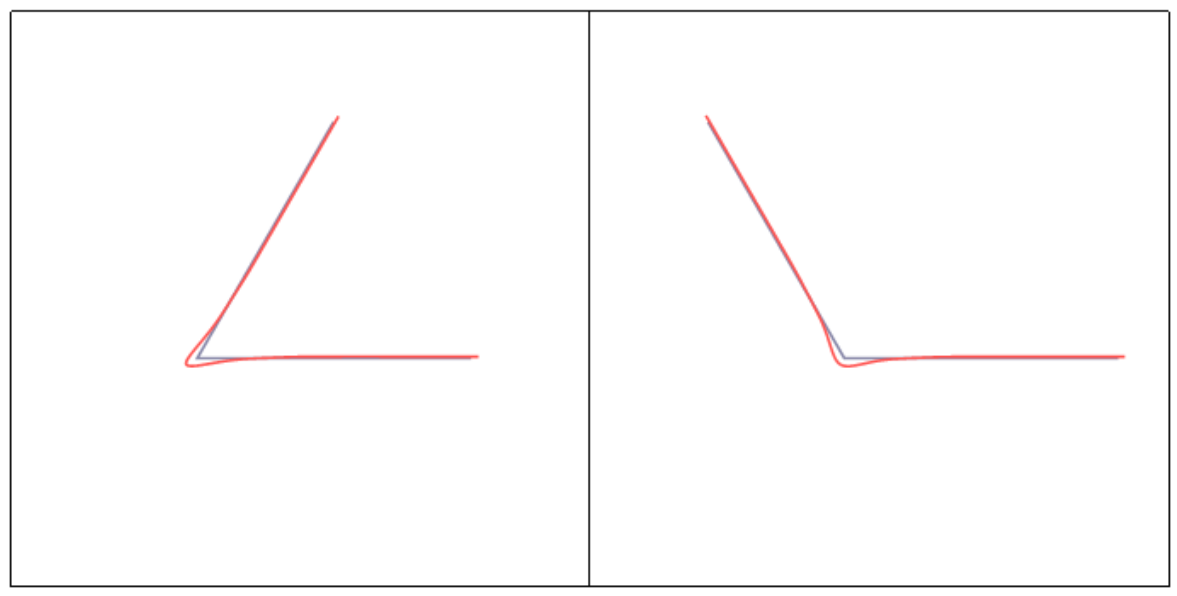

Figure 45 
Yet very difficult, if not impossible, it is to detect this illusionary effect for an isolated angle, especially an acute one. The acute angle has a prominent multi-path aspect, making the angle appear expanded (this multi-path expansion will be discussed in detail in other sections below), which is an opposite effect to the sharpening of the angle. In order for this singlepath effect to manifest itself, special patterns have been devised as in Fig. 46, Fig. 47, and Fig. 48. In Fig. 46 an array of 30 identical acute angles (of 60 degrees) are packed in a row with their sides parallel with each other. In this construct the single-path aspect of the angles in the middle of the row (to be more precise, from the 3rd angle through to the 28th) has been strengthened, and their multi-path aspect inhibited, perhaps completely. It can be observed that the two sides of those angles in the middle of the row appear curved and those angles themselves sharper, just like the Fourier filtered angles shown in Fig. 43 and Fig. 45. (To see the illusion better, the eyes should perhaps be a bit farther away from the figure than is usually suitable for reading text; a distance of half a metre or more may be good.

Alternatively, one can shrink the images appropriately if reading this paper on a computer.) This effect of single-path acute angles is more evident in Fig. 47 and Fig. 48, the former showing 15 rhombuses of the same shape, with the same centre, but of regularly different sizes, and the latter 15 equilateral triangles similarly constructed.

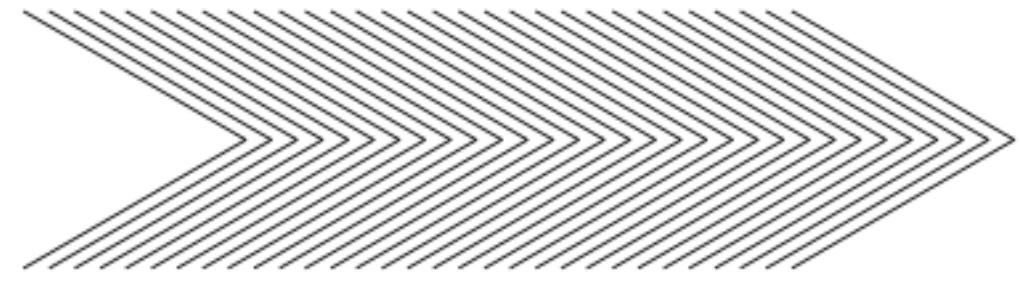

Figure 46

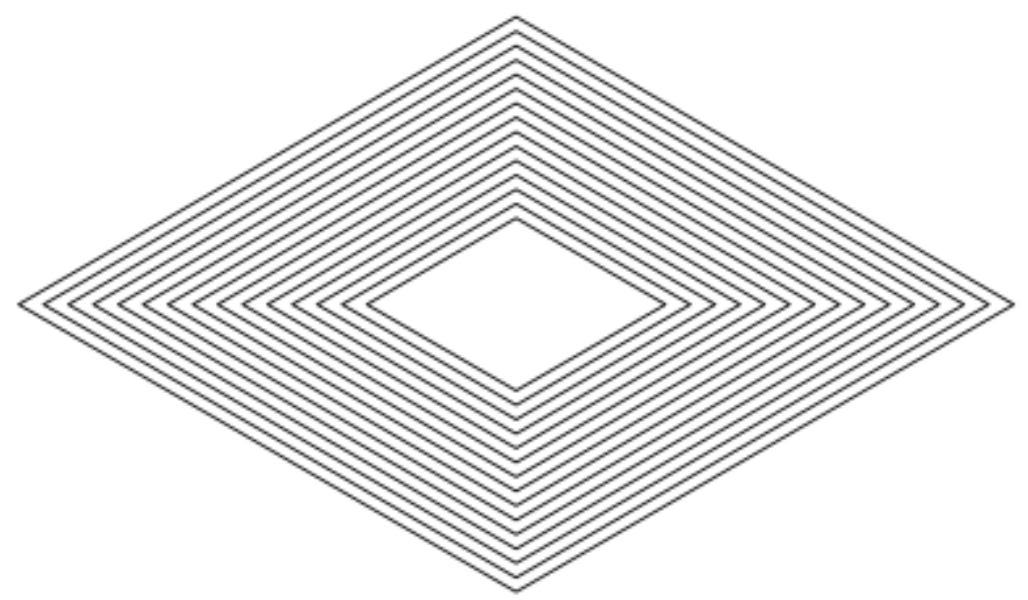




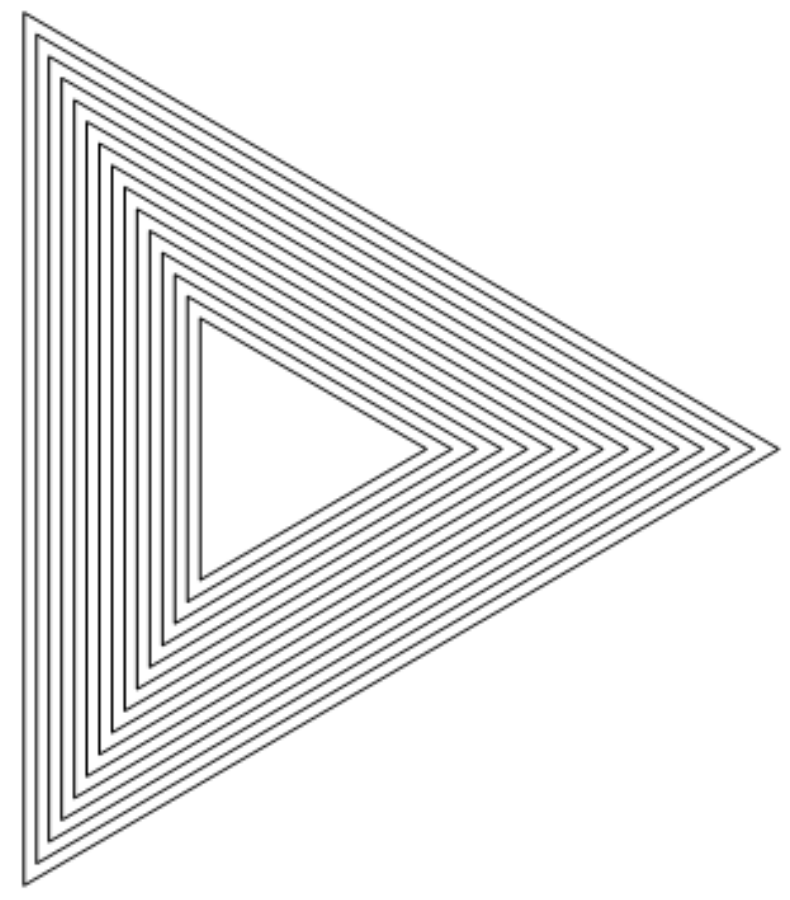

Figure 48

The mechanism of the inhibition, in these patterns, of the multi-path aspect of acute angles is proposed as follows. In Fig. 49, from the middle of the angle row, an angle $<\mathrm{AOB}$ is chosen for discussion. For the visual system to have two virtual particles starting simultaneously from $\mathrm{A}$ and $\mathrm{B}$ and travelling through $\mathrm{AO}$ and $\mathrm{BO}$, respectively, to the vertex $\mathrm{O}$, the visual system has first to detect instantly and definitely that $\mathrm{A}$ and $\mathrm{B}$ are the two ends of the same angle, and also that $\mathrm{AO}$ and $\mathrm{BO}$ are the two sides of that angle. Such detection is impossible, however, within these closely packed angles, if there are absolutely no visual aids present, as in the original figure (Fig. 46). So point A cannot be associated with point B in any way in the visual system, but is probably associated easily with $\mathrm{C}$ and $\mathrm{D}$ instead, end points that are located nearest to it but belong to the previous and next angles. Of all the angles in the row, only the first one and the last one, i.e. the two at the ends of the row, are completely free from this lack of shape identity, and are therefore able to keep their multi-path aspect well; the result is that these two angles do not appear sharpened.

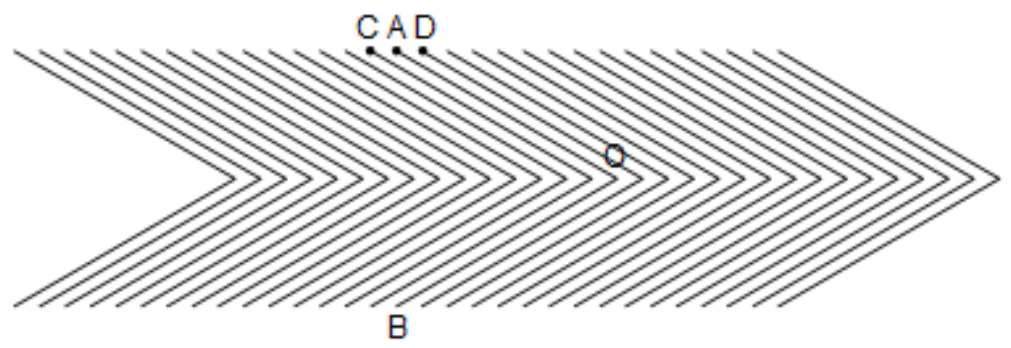


There is an even more interesting phenomenon about these packs of acute angles (Fig. 50 and Fig. 51). If an angle in the middle of the pack is painted a vivid colour (Fig. 50), for example red, and thus its whole shape made distinct to the eye, the effect of sharpening disappears for this angle. Similarly, if an equilateral triangle in the middle of the pack is painted a vivid colour to let the eye identify its three sides with easy and certainty (Fig. 51), the sharpening effects of its three angles disappear. In other words, if the paths of virtual particles for an angle are made definitely unambiguous to the visual system, the single-path visual illusion of the angle is no longer observable.

This recalls the double-slit experiment in quantum physics: when there is no way whatsoever to know which path each electron follows (or, more simply, which slit the electron goes through), an interference pattern of the electrons occurs behind the slits; when, conversely, the path of each electron is able to be determined, the interference pattern disappears.

Although there seems to be very little possibility that the painted-path phenomenon of acute angles here is in any way related to the quantum mechanical double-slit experiment, a degree of similarity between them exists and is perhaps worth noticing.

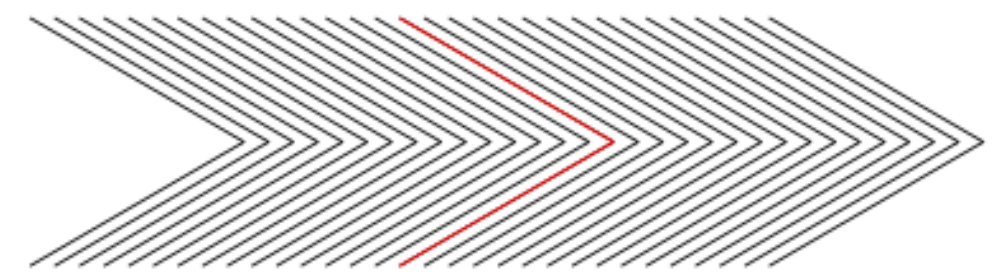




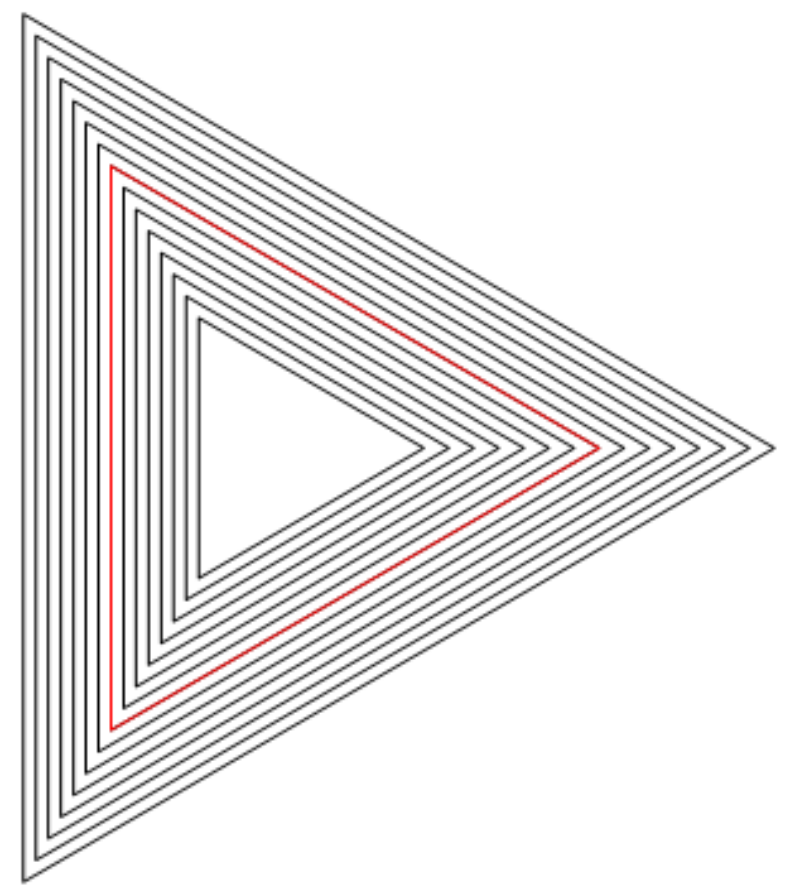

Figure 51

\section{Vertex multi-path figures}

Multiple lines placed and viewed together as a single geometrical construct are treated as motions of multiple particles moving along these lines and interacting with each other in the human visual perception system; such interactions create visual illusions at a level different from that with the single-path illusions. Of great significance and of particular interest to this paper is the vertex multi-path figure, defined earlier. Each path in a multi-path figure still creates, mutually independently, its own single-path illusion. Consequently, the illusions of multi-path figures are a combination of single-path illusions and illusions due to interactions of different paths.

\section{Determination of paths through a vertex}

The problem of what paths the different virtual particles move along in a vertex multi-path figure can be illustrated by a simple example. In Fig. 52a, where two straight line segments $\mathrm{AB}$ and $\mathrm{CD}$ intersect each other at the point $\mathrm{O}$, there are, for the virtual moving particles, these possible paths (each sequence of three capital letters denotes a path): AOB, COD, AOC, $\mathrm{DOB}, \mathrm{AOD}, \mathrm{COB}$, and all the foregoing paths in a reverse direction as well. Now some questions arise. Which of these paths are used by the human visual system? Or more likely, if each of the paths has a weight factor indicating how salient it is in the figure's generation of 
visual illusions, what should these factors be? And, for practical purposes, which of the paths should be retained and which of them are negligible in the simulation of illusions?

It would be impractical, at the current stage of our knowledge of the mechanism of human vision, to try to formulate a definite general principle that determines how the brain chooses what paths for the virtual moving particles to follow when the eye is looking at a vertex multi-path figure of whatever complexity. Nonetheless, a rough principle, whose soundness will be seen in later applications, is this:

The more fluent a path is, the more prominent and influential it is. Here, to be more fluent is to be smoother, to have less bending, and to be less curved. For example, in Fig. 52b, of the paths $\mathrm{AOC}, \mathrm{BOC}$, and $\mathrm{AOB}$, path $\mathrm{AOC}$, the one with the least bending, is the most fluent and therefore the most salient, whereas path $\mathrm{AOB}$, extending an acute angle of 30 degrees, is the least fluent and may well be neglected by human visual perception system. With such a principle, determination of paths or weight factors thereof can be made on an ad hoc basis at least.

The simplest case of vertex multi-path figures is made of two straight lines that intersect at one point (Fig. 52a), namely the vertex in question. In general the two lines are not perpendicular to each other. More discussion of this is in the next section.

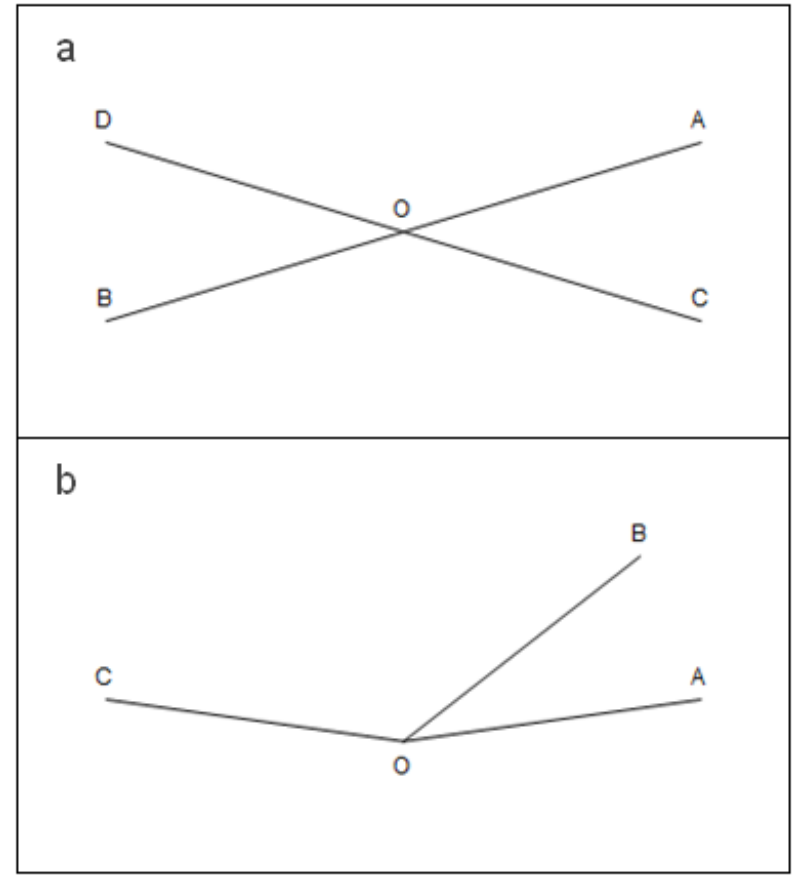

Figure 52

\section{Angle expansion of intersecting lines}

Illusionary expansion of acute angles in the human visual system is believed to be the principal cause of many geometrical illusions. The effect is schematically illustrated, with perhaps much exaggeration, in Fig. 53a: when two straight lines go obliquely across each other (black lines), the acute angles formed by them are seen by viewers as expanded (grey 
lines). With the notion that lines are intrinsically motions to human vision, it turns out that this angle expansion is yet another example of the generalized Gibbs phenomenon.

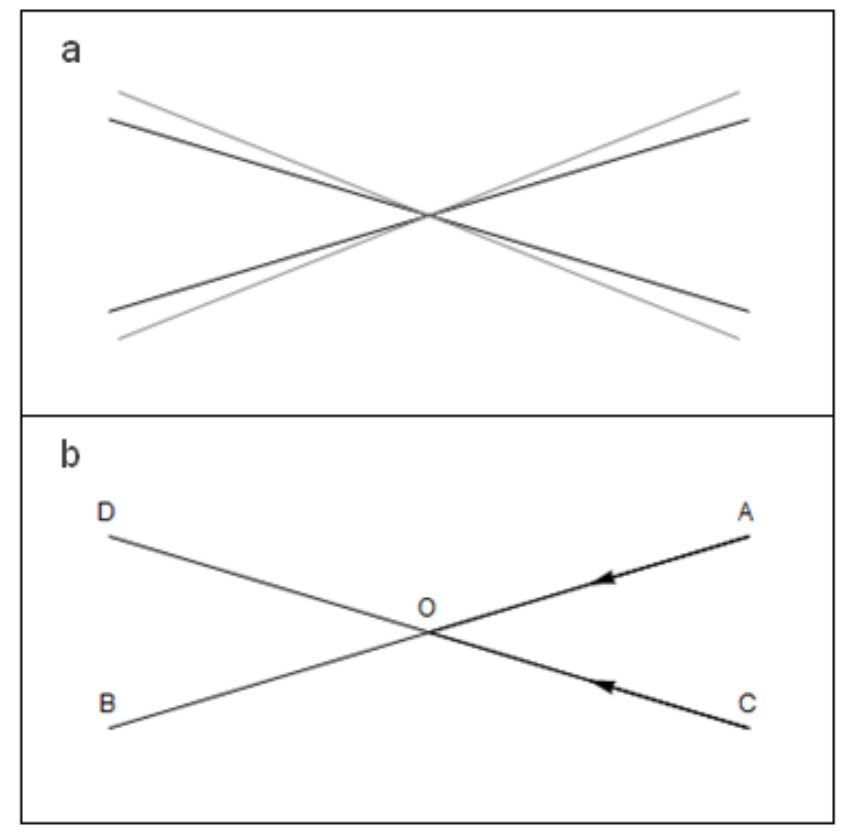

Figure 53

As is discussed right above, in order to analyse the illusion created by this figure, it is needed to determine the paths for virtual moving particles in this situation (Fig. 53b). Straight lines, having absolutely no bending or curvature anywhere, are the most smooth and fluent of all possible curves. Compared with the straight line paths AOB and COD (along with the paths in the reverse direction), all the other paths are bent at the vertex $\mathrm{O}$, and are therefore considerably lacking in fluency, but, among them, at least the obtuse angles, i.e. $<A O D$ and $<\mathrm{COB}$, are still not negligible as paths for the virtual moving particles, especially when they are large (hence constituting quite fluent paths). To the human viewer, these obtuse angles will appear smaller, according to discussions on single-path angles in the previous section.

The angle $<$ AOC obviously acute, as shown in Fig. 53b, do the two straight paths go across each other in the AOB-and-COD fashion (or the direct reverse, BOA-and-DOC), or the $\mathrm{AOB}$-and-DOC fashion (or the direct reverse, BOA-and-COD)? Intuition tells us that our visual system groups the end points $A$ and $C$ together, because they are near, and, similarly, groups $\mathrm{D}$ and $\mathrm{B}$ together, whereas $\mathrm{A}$ and $\mathrm{D}$ are not processed together, for being too far away from each other, and neither are $\mathrm{C}$ and $\mathrm{B}$ for the same reason. So the paths in this case are AOB-and-COD (or, equivalently, the direct reverse of them).

(It is possible, however, that, in the visual system, $\mathrm{A}$ and $\mathrm{D}$ (and likewise $\mathrm{C}$ and $\mathrm{B}$ ) are also grouped together, but considerably less strongly than the grouping of $\mathrm{A}$ and $\mathrm{C}$ (or of $\mathrm{D}$ and B). In that case, paths AOB-and-DOC also exist, but are too weak to compete with the principal paths of $\mathrm{AOB}$-and-COD, and therefore still negligible for practical reasons, unless $<\mathrm{AOC}$ is quite large, e.g. more than 60 degrees.)

To decide further the motions of virtual particles along these two paths, a symmetry property should be taken into account: the line segments $\mathrm{AO}, \mathrm{CO}, \mathrm{DO}$, and $\mathrm{BO}$ are chosen to have 
equal lengths, there being no reason not to adopt this simplification in the geometry here. $\mathrm{AO}$ and $\mathrm{CO}$, in particular, are therefore symmetric about the angle bisector of $<\mathrm{AOC}$.

Thus the virtual particles in this straight line intersection figure move in this way: one particle, starting from end point $\mathrm{A}$, moves at a constant speed along path $\mathrm{AOB}$, and, meanwhile, another particle, starting from point $\mathrm{C}$ at exactly the same time as the first one, moves along path COD, with the same constant speed as the first; the two particles meet at $\mathrm{O}$, the vertex, and then continue to $\mathrm{B}$ and $\mathrm{D}$ respectively.

Now that the raw motions of virtual moving particles have been determined, to be shown next is how the particles interact with each other and thereby generate new particle motions responsible for the expanded acute-angle illusion.

The interaction between the two virtual particles is such that the velocity difference between them gets amplified (hence the need for a velocity-difference amplification factor $\epsilon$ ) when they come very close to each other, in the same way in which a step function's step jump gets amplified in the generalized Gibbs phenomenon (Fig. 9 and Fig. 12). Velocity here has the same meaning as it does in physics, and so it is a vector; and the difference between two velocities is obtained by subtraction of the two velocity vectors. (This velocity difference is just the concept of relative velocity in physics; nonetheless, the former term will be stuck to, since presumably the majority of readers are not familiar with the latter.) Fig. 54 shows, via the solid black curve on the right half of the plot, the velocity difference amplification factor $\epsilon\left(d / d_{0}\right)$ as a function of $d$, the distance between the two particles, $d_{0}$ here being an appropriate length constant, which can for example be thought of as the distance at which two virtual particles cease to interact with each other. Here the dotted curve on the left is symmetrical with the solid curve on the right about the origin of the coordinate axes; the two curves together form the step jump curve in the generalized Gibbs phenomenon (Fig. 12).

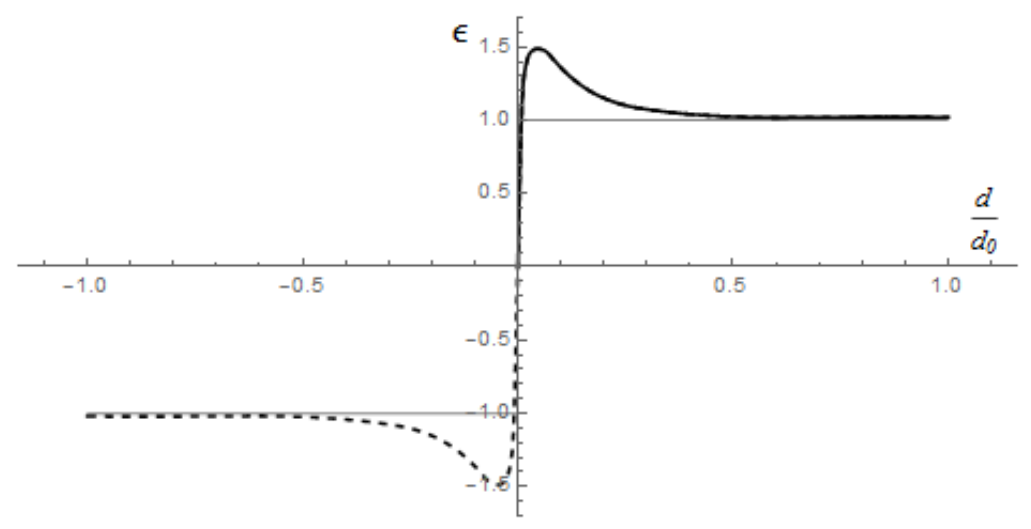

Figure 54

The method of calculation of the illusion of crossing lines is as follows. First, each particle's velocity is written as the average of the two particles' velocities plus half of its velocity difference relative to the other particle.

$$
\vec{v}_{1}=\frac{\vec{v}_{1}+\vec{v}_{2}}{2}+\frac{\vec{v}_{1}-\vec{v}_{2}}{2}
$$




$$
\vec{v}_{2}=\frac{\vec{v}_{1}+\vec{v}_{2}}{2}+\frac{\vec{v}_{2}-\vec{v}_{1}}{2}
$$

Then the velocity differences between the two particles at a certain instant of time $t$ are each multiplied by the amplification factor; thus obtained are the new velocities of the two particles, $\vec{u}_{1}$ and $\vec{u}_{2}$.

$$
\begin{aligned}
& \vec{u}_{1}=\frac{\vec{v}_{1}+\vec{v}_{2}}{2}+\epsilon\left(d / d_{0}\right) \frac{\vec{v}_{1}-\vec{v}_{2}}{2} \\
& \vec{u}_{2}=\frac{\vec{v}_{1}+\vec{v}_{2}}{2}+\epsilon\left(d / d_{0}\right) \frac{\vec{v}_{2}-\vec{v}_{1}}{2}
\end{aligned}
$$

In addition, the distance $d$ between the two particles is certainly a function of time, $t$.

$$
d=d(t)
$$

After that, integration (that concept in calculus) of the two new velocities over time generates the new paths, two curved lines which, despite their slightly irregular shapes, look like the result of expanding the acute intersection angle of the two original lines. This is illustrated in Fig. 55, where the two grey lines are the original crossing straight lines, and the black, slightly curved lines are the computer-simulated illusions of them, based on the theory and method of calculation described above.

Such illusory curved lines can in fact be observed; for example they are fairly obvious in Fig. 56 , where there are two intersections of straight lines. (Yet the two single path obtuse angles $<\mathrm{AOD}$ and $<\mathrm{COB}$ in Fig. 53b, together, actually produce curves of almost the same shape as given in Fig. 55; hence it is still difficult to tell how much of the illusory curve effect in Fig. 56 is multi-path and how much of it is single-path.)

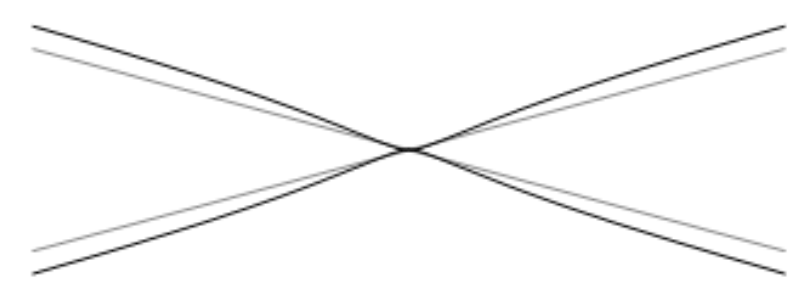




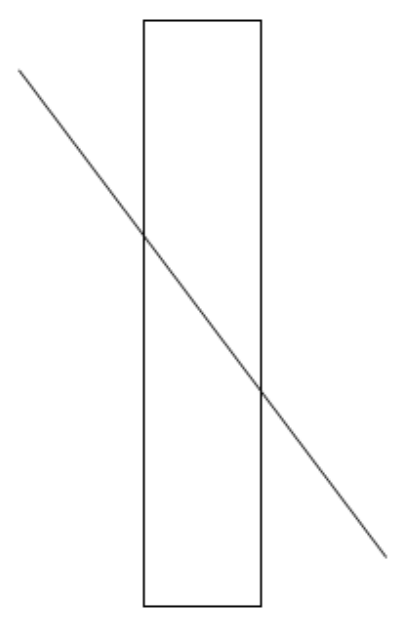

Figure 56

The illusion simulation thus obtained, however, is just part, albeit a very important part, of the story of the illusions of two intersecting straight lines: there is also the illusion of the shrinkage of obtuse angles ( $\angle \mathrm{AOD}$ and $<\mathrm{COB}$ in Fig. $53 \mathrm{~b}$ ), as is mentioned earlier in this section. The point here is that shrinkage of those obtuse angles is equivalent to expansion of the acute angles ( $<\mathrm{AOC}$ and $<\mathrm{DOB}$ in Fig. $53 \mathrm{~b})$. So, these two major illusions, being right in agreement with each other, cooperatively expand the acute angles of the crossing lines.

There is still the problem of how much reverse effect, namely shrinkage of the single-path acute angles ( $<\mathrm{AOC}$ and $<\mathrm{DOB}$ in Fig. $53 \mathrm{~b}$ ), there is in this crossing lines figure. Their competition (in angle shrinking) with the obtuse angles always loses since they are the less salient paths for being the more bent, less smooth ones. Thus the shrinkage of single-path acute angles is totally negligible as a practical simplification.

It is worthwhile to note that an acute angle as investigated in Fig. 43 is hugely different from an acute angle formed by two crossing lines, the former a single-path figure, involving no interaction between virtual particles, but the latter a multi-path figure, where interaction there exists between two virtual particles.

To conclude this section, we look at the situation in which the two straight lines intersect at right angles (Fig. 57). The tendencies of grouping of $\mathrm{A}$ and $\mathrm{C}$, of $\mathrm{C}$ and $\mathrm{B}$, of $\mathrm{B}$ and $\mathrm{D}$, and of $\mathrm{D}$ and $\mathrm{A}$, are all identical, and so the illusionary enlargements of $\angle \mathrm{AOC}, \angle \mathrm{DOA}, \angle \mathrm{BOD}$, and $<\mathrm{COB}$ as multi-path angles are all identical too and thus cancel out. Also, $<\mathrm{AOC},<\mathrm{DOA}$, $<\mathrm{BOD}$, and $<\mathrm{COB}$, each a right angle, are all exactly the same in size, and hence the illusionary shrinkages of them as single-path angles are all exactly the same and thus cancel out. The net result therefore is that there is no illusion of angle expansion or shrinkage when two straight lines go across each other at right angles. 


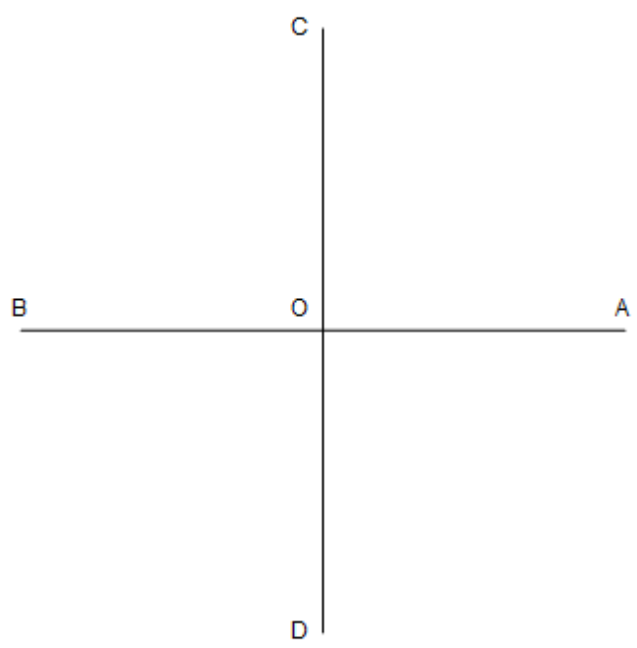

Figure 57

\section{Illusion of a pure multi-path figure}

When two lines (possibly curved) only go near each other somewhere but do not actually meet anywhere (that is, at any intersection or tangent point), illusion may still occur that alters the shapes of the lines. Fig. 58a is an example, where the vertical straight line appears bent towards the circle when getting near to it. In this case there are unquestionably two paths involved in the illusion; so this is a proof of the existence of multi-path illusions. The simulation of this illusion is the same as in the last section, using the velocity difference amplification factor, except $(a)$ that here the distance between the two virtual particles never reaches zero, and $(b)$ that the two virtual particles along the straight line and the circle respectively need to be going in the same direction, especially both downwards, when in proximity, and to be so synchronized that the straight-line particle reaches the mid-point of the straight line segment at the same time as the circle particle reaches the leftmost point on the circle. Fig. 58b is a two-straight-line version of Fig. 58a, appended here for a better experience of such an illusion. 

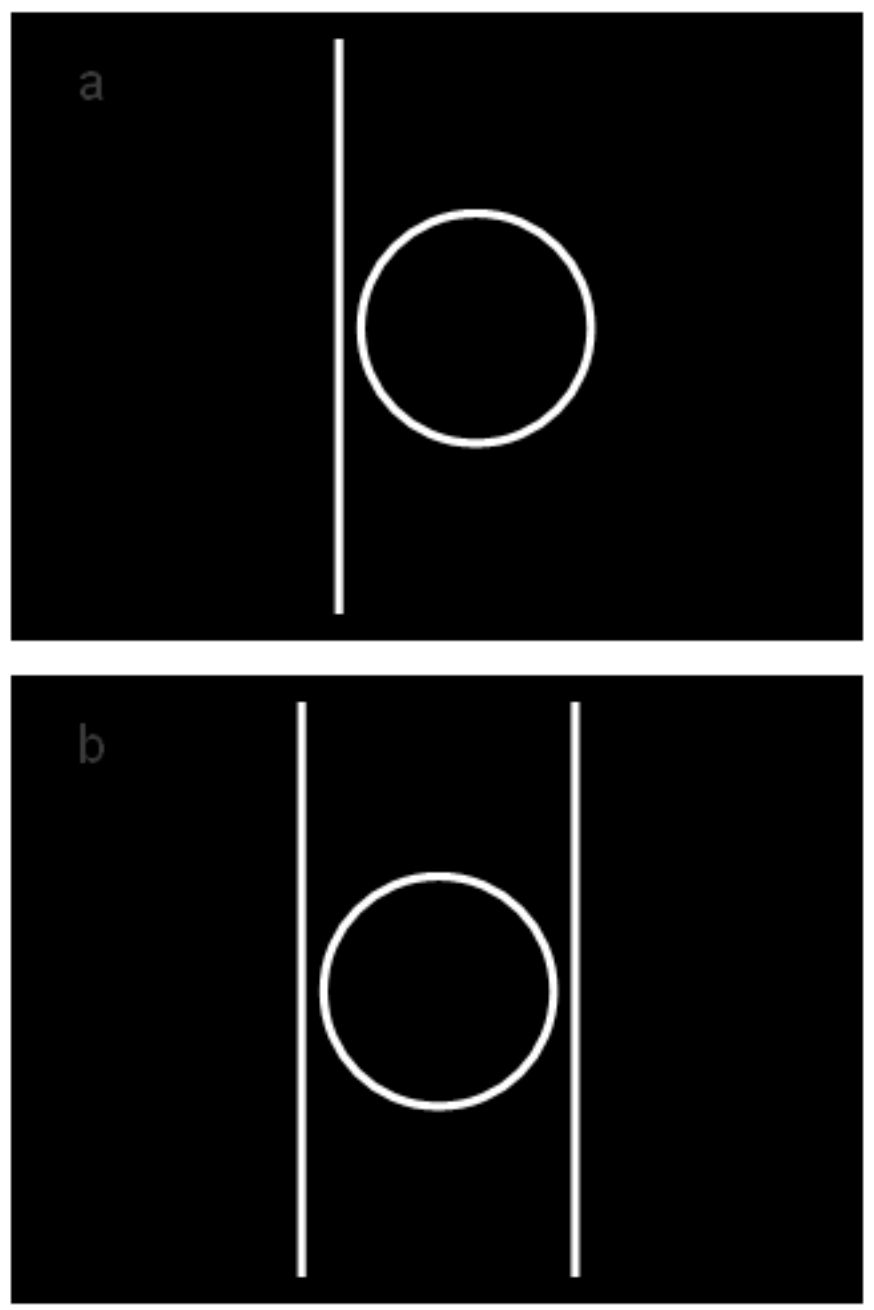

Figure 58

\section{The Poggendorff illusion}

As shown in Fig. 59 (which comprises, from left to right, Fig. 59a, 59b, and 59c, the labels $a, b$, and $c$ having been omitted so as not to pose distraction to viewing), the Poggendorff illusion, in its most common form, is the misjudgement, by the human visual system, of whether two oblique parallel straight line segments separated by two vertical straight lines (or a broad vertical stripe) are on the same straight line, i.e. lined up with each other. In Fig. 59b, the two oblique straight line segments appear to the visual system to be on the same straight line; in actuality, they are not. It is in Fig. 59a that the oblique line segments are on the same straight line, although they do not appear so to the human eye. Intended to show the illusion more vividly, Fig. 59c is the superimposition of the two figures on the left; here, of the two oblique line segments to the left of the vertical lines, the viewer may want to ask which one is on the same straight line with the oblique line segment on the right. The answer is just the opposite of the judgement of the eye, as can be verified with a ruler. 

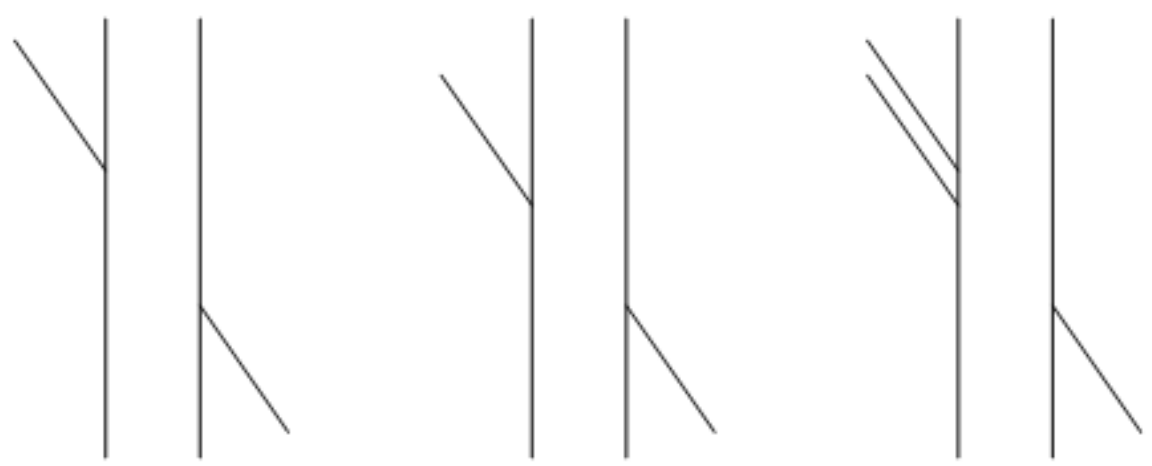

Figure 59

This illusion has most often been explained by the proposition that the acute angles in it are expanded by the human visual system. Such a view is in complete agreement with the dynamic interpretation of lines as paths of virtual particles, which gives an analysis of the problem as follows.

First it needs to be presented how that expansion of acute angles is able to cause the anomaly observed in the illusion. Fig. 60a is a labelled version of Fig. 59a, namely the original geometric configuration; so here, needless to say, DP is lined up with AO. The acute angles involved in this case are $\angle A O B$ and $<D P F$ in Fig. 60a. Fig. 60b shows the effect of the expansion of these two angles. The solid grey lines are the original configuration (namely Fig. 59a), where the oblique lines are lined up with each other. The two black lines represent the respective oblique sides of the angles $<\mathrm{AOB}$ and $<\mathrm{DPF}$ after they have been expanded; the dashed grey line indicates the infinite straight line to which the black line on the right belongs. The shifting upward of the left black line segment from the dashed line (i.e. the straight extension of the right black line segment) is obvious, hence the Poggendorff illusion.

a

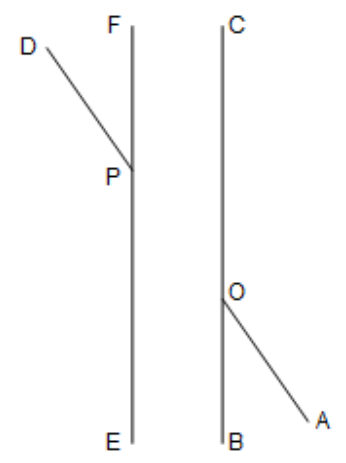

b

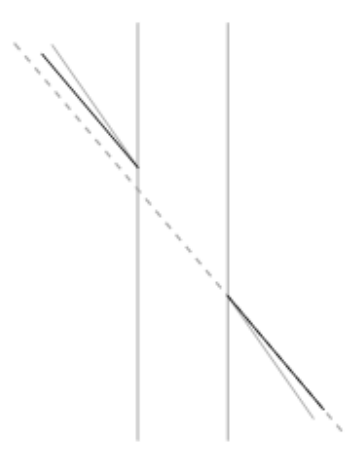

Figure 60

Here then is how expansion of acute angles arises in the Poggendorff illusion. Since this figure (Fig. 59a or, equivalently, Fig. 60a) is symmetric about its centre, it suffices to talk about the right part only. At the vertex point $\mathrm{O}$, there are three branches $\mathrm{OA}, \mathrm{OB}$, and $\mathrm{OC}$; as a single-path vertex can have only two branches, the vertex $\mathrm{O}$ here is a multi-path vertex. It is 
evident that point $\mathrm{C}$ cannot be grouped together with either $\mathrm{A}$ or $\mathrm{B}$, for being too far apart from them, whereas $\mathrm{A}$ and $\mathrm{B}$ are so close together that they are grouped together in the visual system as the starting points of two paths. Consequently, the two paths are BOC and AOC (as shown in Fig. 61a and Fig. 61b respectively). Thus $<\mathrm{AOB}$ is the multi-path angle in this question and is the same as $<\mathrm{AOC}$ in Fig. $53 \mathrm{~b}$ in the previous section. Such is one of the reasons why $<\mathrm{AOB}$ in Fig. 60a is expanded. There is another one, which is probably more important.

a

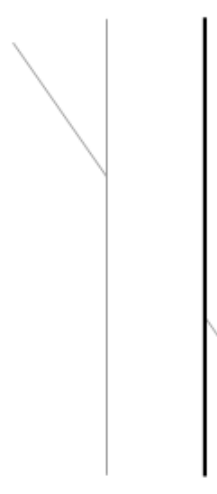

b

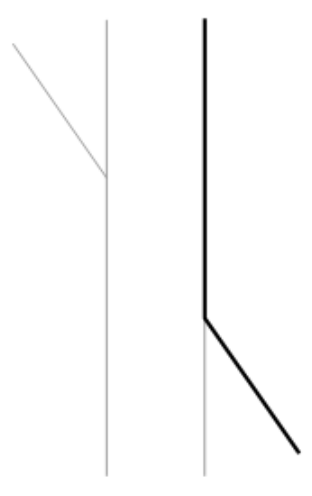

Figure 61

It has been pointed out that each of the single path angles in a multi-path figure generates its own illusion too; accordingly, in the Poggendorff illusion, paths AOC and AOB (Fig. 60a) should be investigated. As single path angles, $<\mathrm{AOC}$ and $<\mathrm{AOB}$ are both subject to illusionary shrinkage, but their deformation effects are directly opposite to each other on the apparent direction of the line segment OA. Path AOC, however, forming an obtuse angle, bends less, is more fluent, and hence has greater importance and, mathematically, more weight, than path $\mathrm{AOB}$, which forms an acute angle. So the shrinking competition between $<\mathrm{AOC}$ and $\angle \mathrm{AOB}$ is won by the former, with the simple net result of the latter, that acute angle, being expanded.

In summary, the illusionary expansion of the multi-path acute angle and the shrinkage of the adjacent single-path obtuse angle are both constructively involved in the Poggendorff illusion. It is yet difficult to decide their respective weights in the overall effect of the illusion.

\section{Other illusions similarly produced}

There are other well-known geometrical illusions that are readily explainable by the combined action of the expansion of a multi-path acute angle and the shrinkage of the adjacent single-path obtuse angle, notably the Hering illusion and the Zöllner illusion. Of the Hering illusion is Fig. 62, in which the two horizontal straight lines, accompanied by the radial lines, appear as if the upper one were bowed upwards and the lower one downwards. As a modified version of the Zöllner illusion, Fig. 63 consists of some parallel horizontal lines crossed with many short oblique lines that alternate their directions for different horizontal lines. Strangely these horizontal lines do not appear parallel or horizontal, but the fact is that they are. 


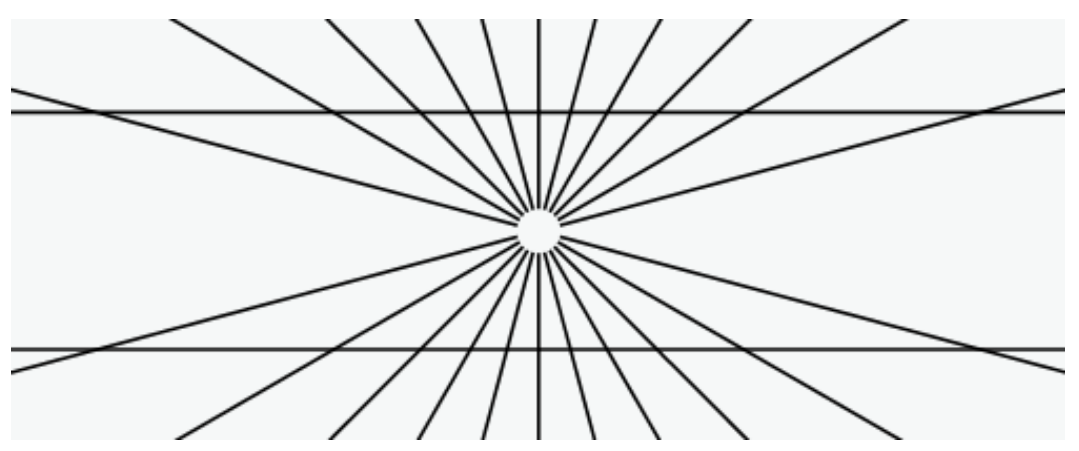

Figure 62

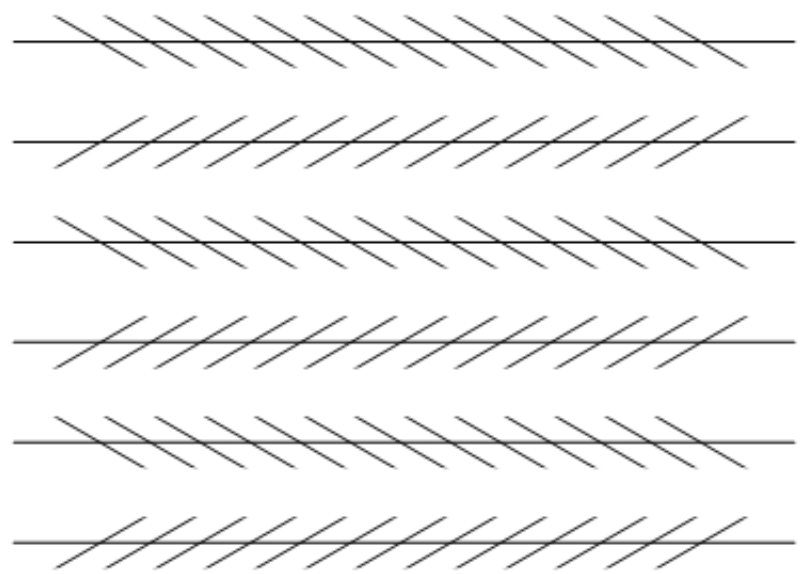

Figure 63

Omitted are the details of explanation.

\section{Remarks on some basic concepts}

Thus, out of all the preceding discussions, a duality property of vision starts to emerge, that, for raster brightness or colour illusions, the image should be conceived as being composed of two-dimensional sinusoidal waves, but, for geometrical illusions with lines, the image should be considered as being composed of virtual moving particles on a two-dimensional plane. It reminds one of the fact that waves and particles are two basic concepts in physics. A pebble thrown into the air, following a parabolic curve, is a particle, whereas a sound travelling in the air is a wave. Matter, according to quantum mechanics, exists both as waves and as particles; an electron, for instance, sometimes acts as a wave and sometimes as a particle. Besides those associations, there is a picture, as it were, worthy of mentioning: the virtual moving particle 'flowing' in a line, in the previous discussions, is guided by the 'path waves' which are the outcome of the Fourier visual filtering of its path.

To use one virtual particle for one path, with all particles (in a multi-path figure) moving at the same constant speed, is the simplest possible method of modelling the illusionary paths; the validity of it is determined by whether the result so obtained fits the illusion phenomenon in question. Yet it is certainly more plausible that each path is run along by a stream of particles just as a flow of water is a stream of 'water particles' moving along the 
stream. Also, the same constant speed for all virtual particles is sure to prove to be too drastic a simplification; for instance, in the Hering illusion (Fig. 62), the virtual particles moving along the many radial lines do not seem to have the same speed as one another, because the outer, more oblique radial lines seem to possess more 'momentum', so to speak, than the inner, more vertical radial lines. Moreover, in a complex figure where a lot of paths are involved, such as the Hering illusion figure, path interactions are conceivably not just within each local vertex construct.

When, more generally, the thickness of the lines involved cannot be ignored, possibly even changing along the path, which is a situation that the present paper does not deal with, a line has to be mathematically represented by a field of moving particles, the model resembling a fluid continuum, consisting of 'fluid particles', in fluid mechanics. Furthermore, for certain complex curve shapes, a two-dimensional field of virtual particles will be insufficient; a three-dimensional field, instead, is necessary, because the brain no longer, it is here proposed, processes in a two-dimensional manner shapes that are beyond a certain structural complexity or subtlety. This, in addition, is how Chinese calligraphy and certain crucial aspects of Chinese painting may be scientifically studied, if such a study is possible, in the distant future.

\section{Vector brightness illusions}

We have discussed, as two substantial parts, firstly 'raster' illusions by brightness (or colour) patterns that are not sensitive to the pattern's geometry (e.g. Fig. 30 and Fig. 31), and secondly geometrical illusions by lines and shapes where only the shape of the figure is altered by the visual system and where the brightness pattern is of no relevance whatever. It is now time to turn to those visual illusions that involve both aspects, namely, brightness illusions which have a direction, and are crucially dependent on the figure's geometry, and which can be called vector brightness illusions..

Here the concept of the speed of the virtual moving particle flowing along a line, either straight or curved, will play an important role. The virtual moving particle of a straight line, it is reasonable to assume, possesses higher speed than that of a curved line, the two lines being thin and equal in thickness: the more curved the line, the lower the particle's speed (Fig. 64). A more accurate version of the assumption is that the particle's speed on an arbitrary curve (generally with non-constant curvature) is a monotonically decreasing function of its curvature along it. Such an assumption is based primarily on the artistic experience that a straight line visually arouses a feeling of faster movement than does a curved line. 


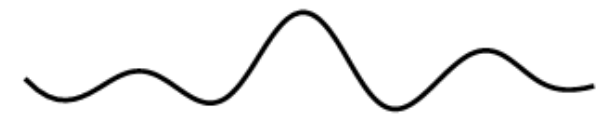

Figure 64

Nonetheless, it may occur to some people that in Fig. 64 an obvious sense of movement is given by the curved line, but not by the straight line, in which the virtual moving particle is said to possess a higher speed. So it should be pointed out that the 'movement' felt to be displayed by a line is often not the speed of the virtual moving particle involved, but the rhythmical change of its velocity (here, change in direction counts as well). Thus, if the curved line in Fig. 64 is replaced by a zigzag line of a similar shape and of the same span, made up of a series of joined straight line segments with sharp turns, a feeling of movement is produced too, though far less agreeable than given by that smooth curve.

A further assumption is necessary: that the virtual particles somehow influence the brightness (and also colour) information of the path which they follow, and the higher the speed of the virtual particles is on a certain path, the more prominent the effect of visual filtering on brightness (i.e. the brightness illusion) will be along that path. Special geometric constructs can be devised to test these two assumptions as a whole.

\section{Anisotropic visual filtering}

There are, it must now be assumed, virtual moving particles at all places of an image, whether it is a point on a line or a spot in the middle of a disc, and these virtual moving particles can be thought of as air molecules in a room. An image without pronounced straight-line patterns can be thought of as being analogous to a room of static air without any currents in it, in which the huge number of air molecules move randomly in all directions; it is therefore subject to visual filtering with isotropic filters, which has been the case with all the treatments of brightness illusions and colour illusions before.

By contrast an image with pronounced straight-line patterns can only be thought of as being analogous to a room in which there are apparent air currents, and the virtual moving particles as being analogous to air molecules moving along in those currents. In this case the virtual particles moving in a straight current possess large speed, and go in the direction of the flow of the current. (Such analogies with air molecules in a room are intended just to be practical for present purposes, if not appropriate enough, as the average speed of the random motion of air molecules is extremely high, approximately $500 \mathrm{~m} / \mathrm{s}$, but the average distance they can travel in a straight line without collision with others is just $0.1 \mu \mathrm{m}$.) Hence, according to the assumption two paragraphs back, the effect of brightness illusion will be stronger along this current, making necessary the adoption of a two-dimensional Fourier 
filter that has a more narrow shape in the direction of the current, namely an anisotropic filter.

Specifically, a straight line with a certain thickness will direct virtual moving particles in it to move inside and along this straight line at relatively fast speed, and also direct virtual particles near to it and belonging to the background to move straight along but beside this line at relatively fast speed. Further, the fast movements will not be stopped by a small gap or obstacle.

\section{The straight line gap illusion}

Fig. 65 shows eleven black horizontal straight lines with short gaps in them; the illusion is that fairly bright line segments can be seen to fill the gaps between horizontally consecutive black line segments, namely, to connect them. The other aspect of this illusion is that the white background of the image is no longer purely white, but slightly grey except at those bright gaps. When the black lines become wavy, however, the illusion disappears (Fig. 66). On the other hand, the illusion persists even when the gaps are quite large (Fig. 67).

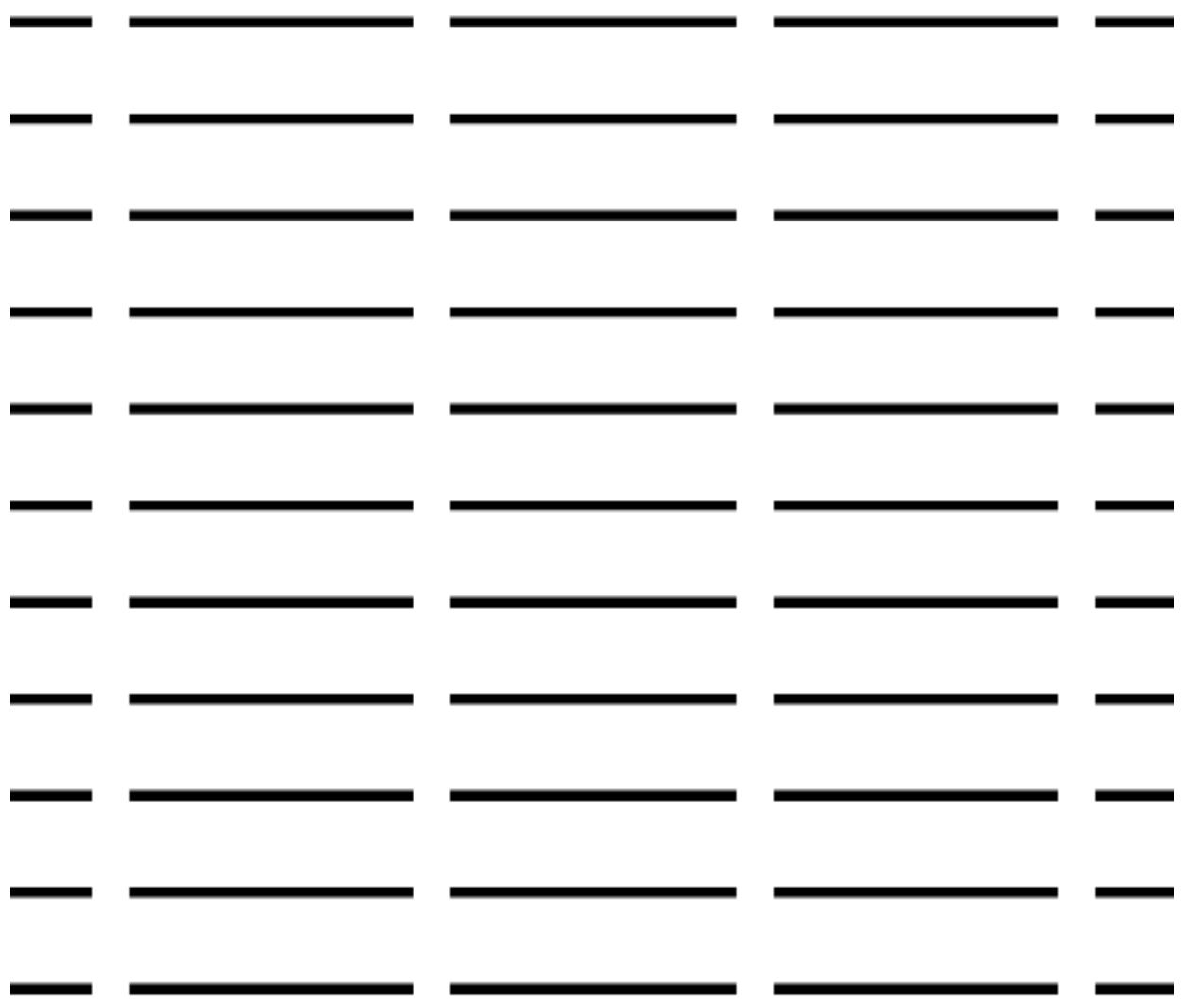




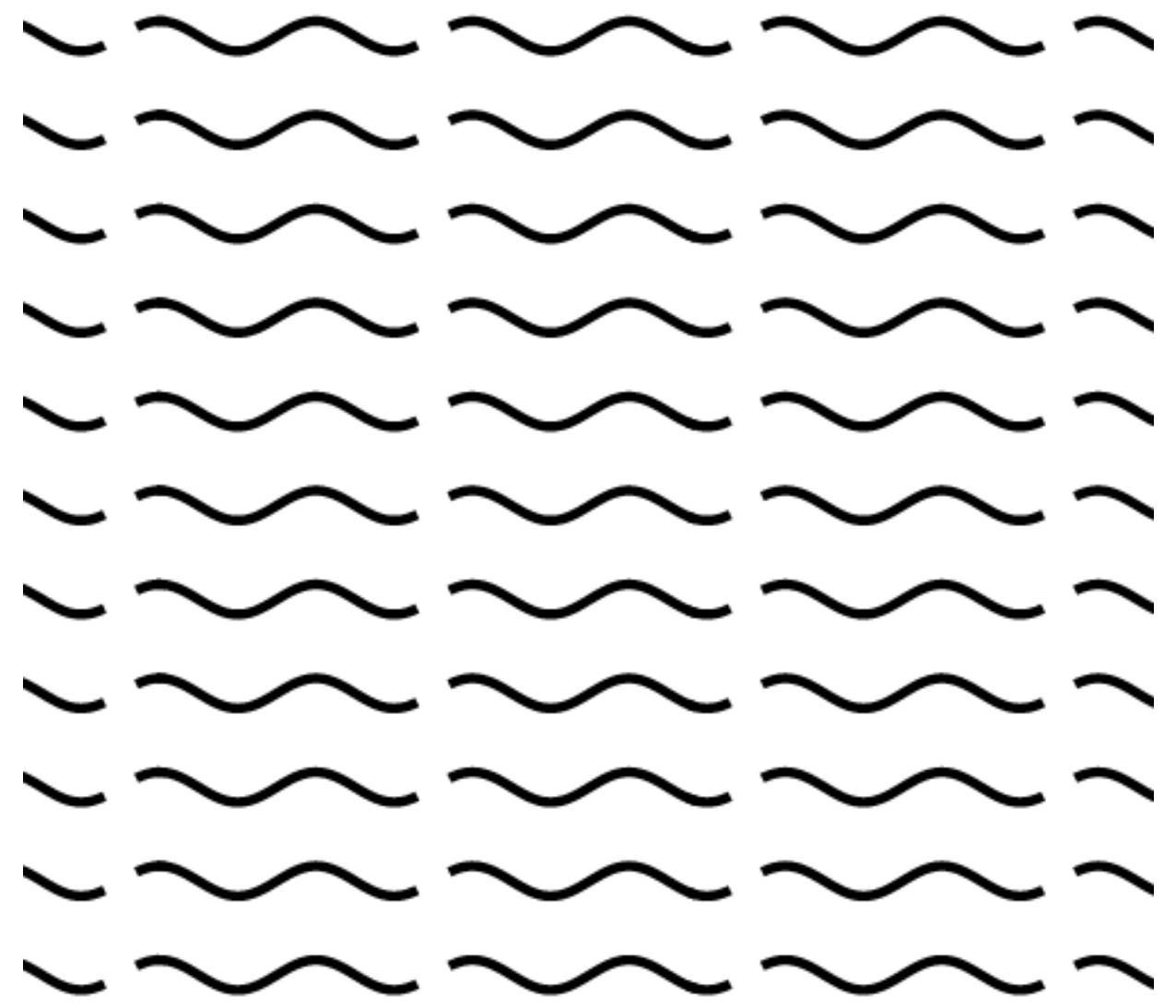

Figure 66 


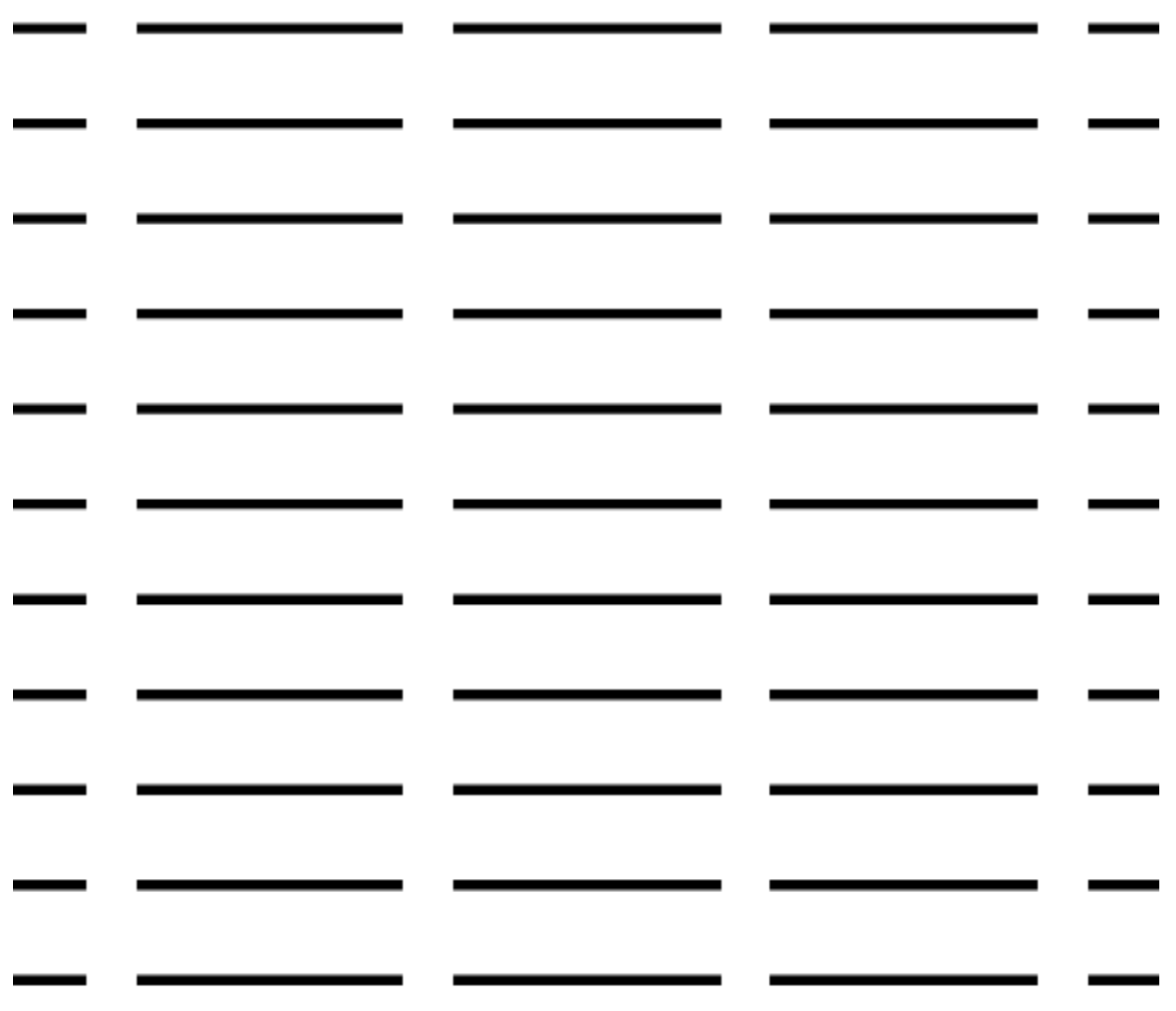

Figure 67

It is evident that in the original pattern, i.e. when the lines are straight, there is a strong, horizontal flow of virtual particles which covers the whole picture, forming either the main or the only current in it; therefore an anisotropic filter which is much narrower in the horizontal direction than in other directions is needed for brightness filtering. In the wavy pattern (Fig. 66), however, the straight, fast horizontal flows of virtual particles are destroyed; as a result, the strong illusion-inducing anisotropic filtering is no longer applicable in this case, hence the disappearance of the bright gap illusion.

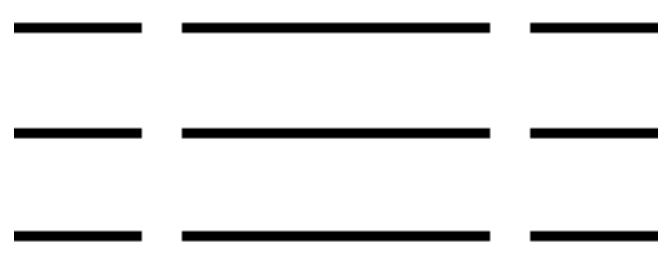




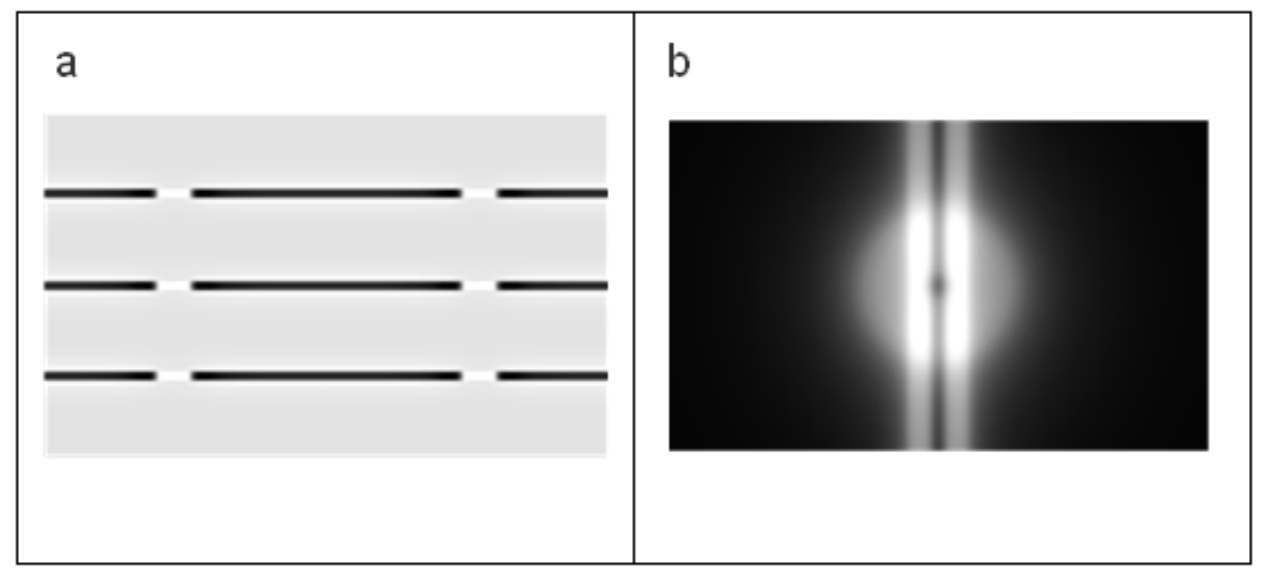

Figure 69

A simplified but equivalent version of Fig. 65, Fig. 68 is used for visual filtering on the computer. Fig. $69 \mathrm{~b}$ is a two-dimensional greyscale representation of the anisotropic filter used in the filtering (where the greyscale value at a certain point equals the value of the filter at that point), and Fig. 69a the filtering result, which correctly and manifestly depicts the straight line gap illusion. This anisotropic filter, specifically, is the superposition of (1) an ordinary isotropic filter whose cross-section curve looks broad and which does not produce evident filtering effect at all and is almost negligible, and (2) an anisotropic filter which looks quite narrow in the horizontal direction and produces strong filtering effect only in this direction. The maximum value of this anisotropic filter can be greater than one, and rescaling of the brightness data of the filtered image is needed, as in all visual filtering experiments, to make the final filtered image look realistic enough.

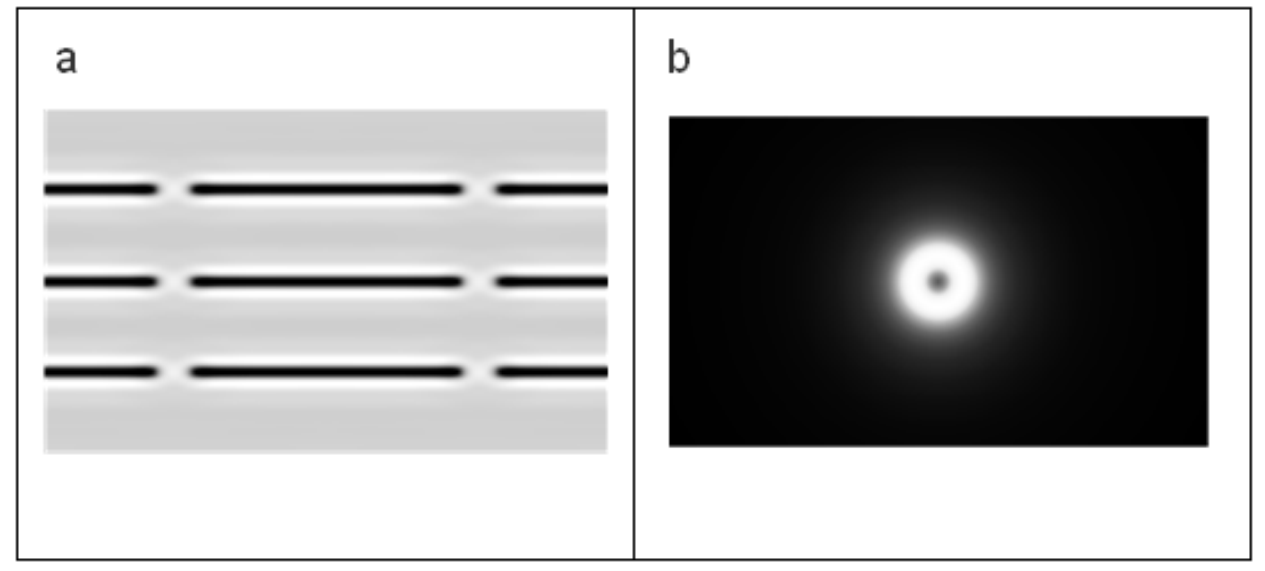

Figure 70

As a comparison, Fig. 70a is the result of filtering the same original pattern with a normal isotropic filter (Fig. 70b). The problem with this result is that although a small bright area appears at each end of a line segment (namely, within every gap), it exists as part of the bright contour coating the whole black line segment, therefore joining the gap very poorly, and that the other parts of the bright contour are of comparable scale and brightness. This is decidedly wrong.

From the significant difference between Fig. 69a and Fig. 70a can be drawn a noteworthy conclusion. In the mammalian visual cortex, simple cortical cells are known to exist that 
respond strongly only to straight lines in the visual field ${ }^{4}$. Each member of such cells is thought to receive information from ganglion cells whose individual circular centresurround receptive fields are well aligned and form a straight line in the retina. Simple cortical cells together with their ganglion cells, if so constructed, would produce, contrary to observation, a raster brightness illusion like that shown in Fig. 70a, rather than the straight line gap illusion.

Incidentally, there is a slightly different modelling of this gap illusion. The superposition used above of an ordinary isotropic filter and an anisotropic filter is one happening at the filter level. Approximately equivalent to that is one happening at the image level, which, to be precise, is a superposition of (1) the insignificant brightness pattern obtained by filtering the raw image with the weak isotropic filter, namely the disc-shape part of Fig. 69b, and (2) the brightness pattern obtained by filtering the raw image with the purely anisotropic filter, namely the two-vertical-bars part of Fig. 69b. Conceptually, part (2) can in essence be simplified into a situation in which a one-dimensional filter is applied to a one-dimensional 'image' consisting of a single black straight line with a white gap in it. Thus we get a onedimensional brightness illusion, which is most likely to be neuroscientifically more simple than any ordinary two-dimensional brightness illusion.

\section{The disc-on-straight-line illusion}

In Fig. 71, identical small white discs are placed evenly on white horizontal straight lines. Inside any white disc an illusionary horizontal grey bar can dimly be seen to go through it, connecting the white lines on the two sides of the disc. Again an anisotropic filter (Fig. 72c) like that in Fig. 69b is used to filter the image, and the result is shown in Fig. 72b. 


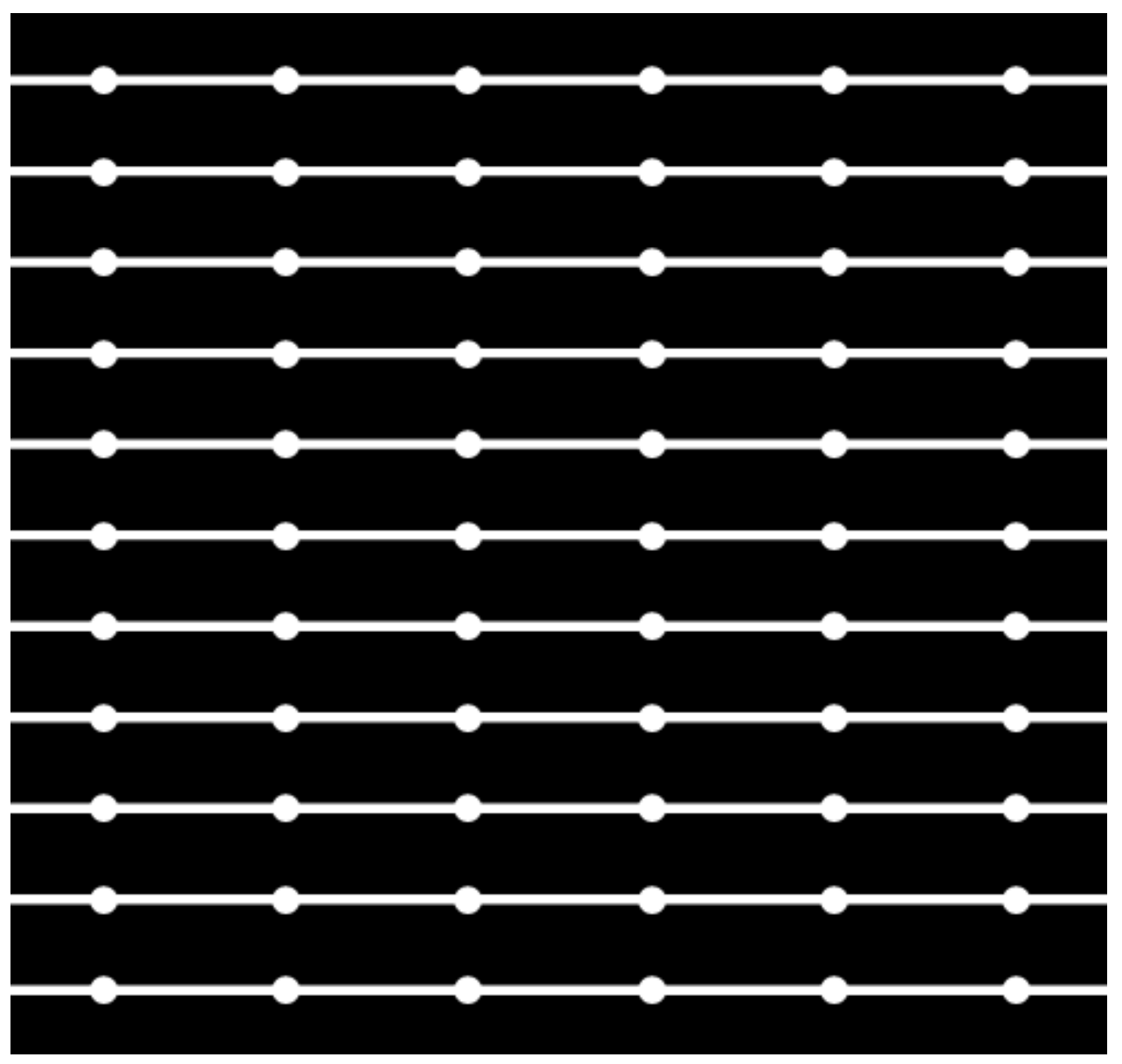

Figure 71

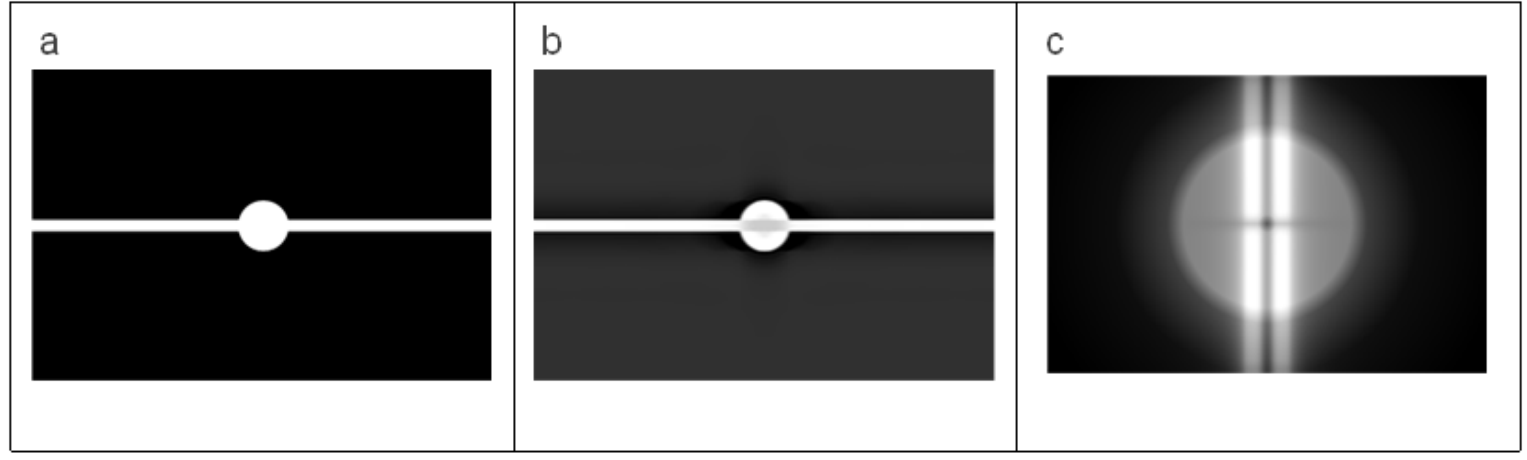

Figure 72 


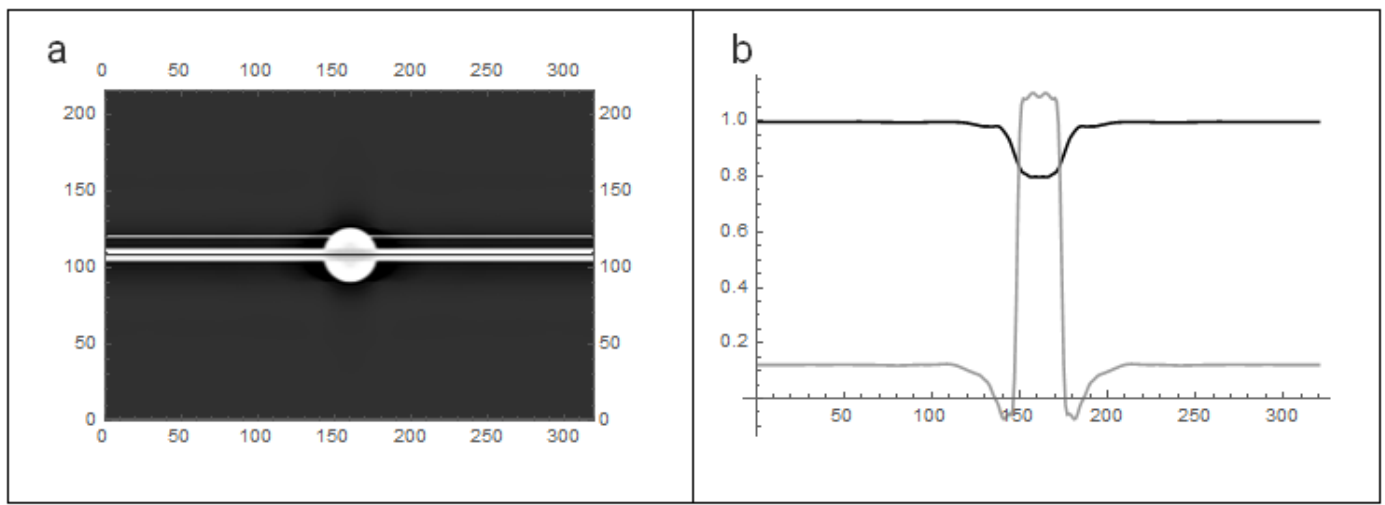

Figure 73

In addition to the illusionary grey bar, there is in fact another illusionary effect here to observe, less noticeable but equally important: the brightness of the upper and lower parts of the disc, which are not covered by the illusionary grey bar, is enhanced. This is demonstrated clearly in Fig. 73. Fig. 73a shows the two horizontal sample lines along which the brightness data of the filtered image are gathered; Fig. $73 \mathrm{~b}$ is a plot of the brightness values along these lines, as two functions of horizontal pixel position, the black curve corresponding to the horizontal line going through the middle of the white line in Fig. 73a, and the grey curve to the horizontal line going through the upper part of the white disc. From Fig. 73b we see that if the filtered image is scaled such that the brightness of the horizontal white line (to be white is to have a brightness value of 1) is still 1 after the filtering process, then the brightness of that upper part of the white disc will manifestly exceed 1 (see the middle part of the grey curve). That means that the upper part of the white disc appears brighter to the human viewer than the horizontal white line. (This second effect is crucial to the mechanism of the spokes illusion, to be discussed next.) These two illusionary effects together make the discs, though completely flat in form, look somewhat like white, translucent beads.

\section{The spokes illusion}

In Fig. 74 is yet another pattern that creates what can be well explained by the notion of vector brightness illusion. In this image, the centre of the white spokes (straight, radial lines of constant thickness throughout) is a white disc of the same size and the same colour as the white disc on the right of the image; however, the left disc, serving as the centre of the spokes, looks much brighter than the right disc, and even shining if the number of spokes is large enough. Peripheral vision is required for a clear experience of the illusion, and is easily performed by gazing at a dark area that is equally distant from these two discs. To better view the shining effect of the disc at the centre of the spokes, one can look at an area (as opposed to a precise point) half the length of a spoke away from the central disc. (If possible, it would be even better to change the background colour of this PDF document temporarily from white to a dark colour.) A variant of this pattern is shown in Fig. 75, where the central disc of the spokes looks evidently much brighter than any of the spokes, despite being of the same white colour. 


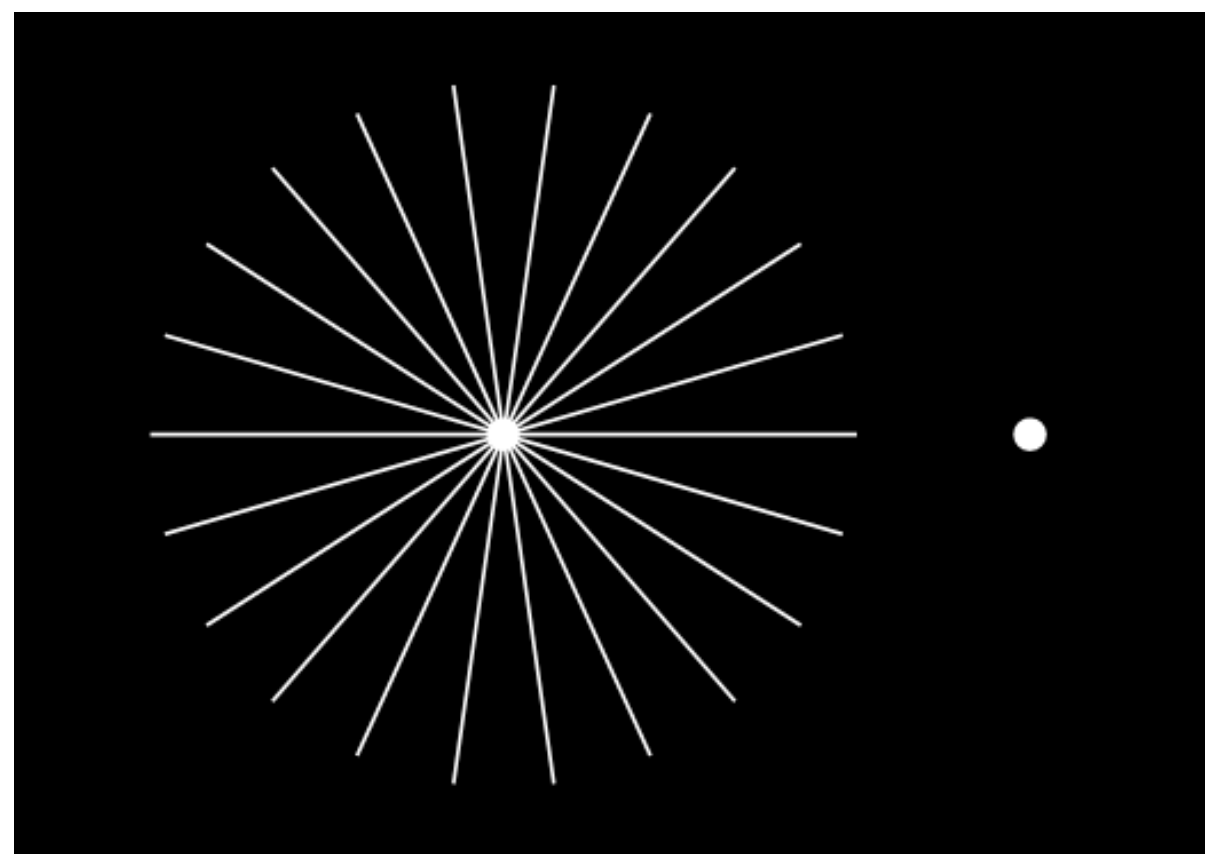

Figure 74

Moreover, in the central disc of a spokes illusion figure (Fig. 74; but not Fig. 75 as in its normal size), there is also a secondary but equally significant effect to observe. When appreciating the brightness of the central disc, we can vaguely see that the central part of the disc is more or less darker than the outer parts of the disc. This less obvious effect will also be explained below.

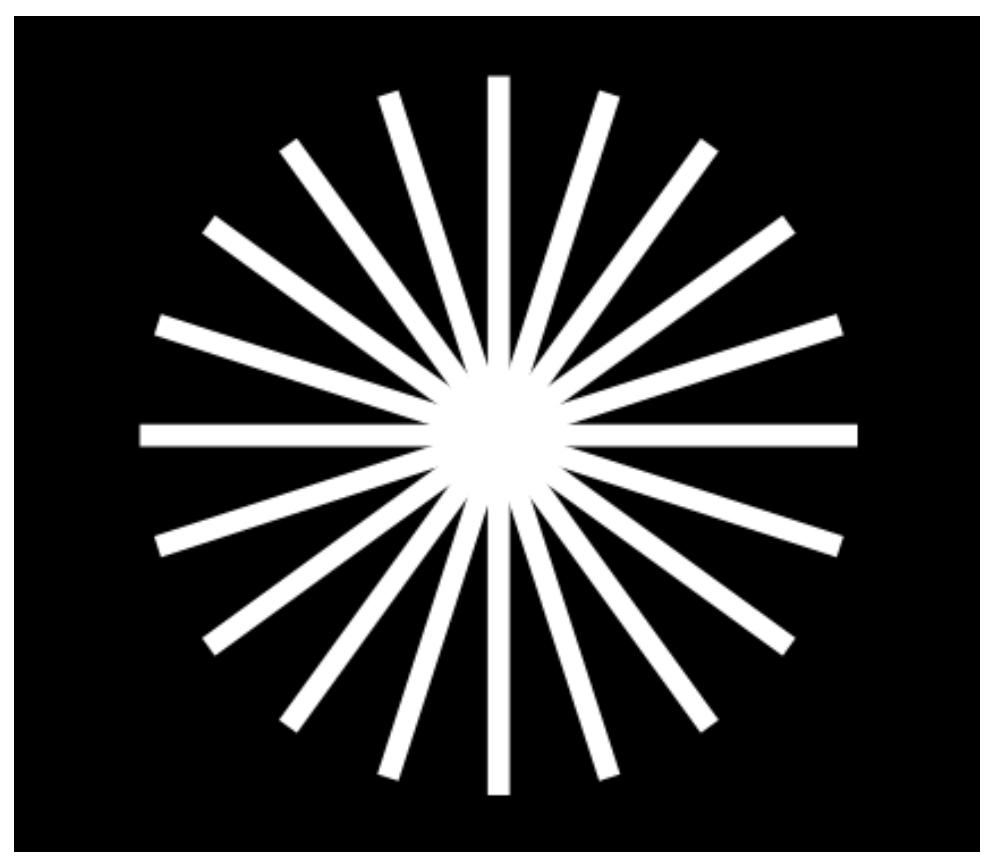

Figure 75

Fig. 76a, -b, -c, and -d show the brightness patterns of the central disc in the spokes illusion when there are $2,6,12$, and 18 spokes respectively (the spokes are not drawn). In all the figures of spokes illusion given in this paper, the spokes are always in pairs such that each pair comprises two opposite spokes which are on the same straight line. According to the 
discussion of the disc-on-straight-line illusion, especially the result contained in Fig. 73, each pair of spokes slightly brighten the central white disc except in the 'grey bar' part, which is very slightly darkened by them instead. The superposition of such effects produced by all the different pairs of spokes present is exactly what is illustrated in Fig. 76.

In any of the four panels of Fig. 76, the brightness of any point on the plotted central disc is not itself intended as the perceived brightness of that point in the illusion, but instead helps to indicate the perceived brightness thereof according to the legend for that disc, that vertical bar, marked with numbers, to the right of it. Along the legend, the numbers specify the degrees of brightness observed by the human visual system for all the greyscale values found in the disc, with the white ceiling of the legend corresponding to the white parts in the disc and representing a perceived brightness that can conspicuously exceed 1. Moreover, the degree of perceived brightness of the white spokes (outside the white disc but not plotted here) is assumed to be 1 in this illusion.

Thus, in Fig. 76d, for example, which involves 18 spokes, the outer part of the disc is, according to the legend, roughly 1.7 times as bright as the spokes, hence the shining effect. This disc figure itself, it should here be noted, is not exactly how the illusionary disc looks to the human eye; nor is any other disc in Fig. 76. As an illustration, in Fig. 76d the central part of the disc is quite dark, but has in spite of that a brightness degree of 0.65 , which means that this part looks grey to the eye. This grey centre is not difficult to observe (peripheral vision needed) when the central disc is of an appropriate size, as in Fig. 77. (In addition, the degree of darkness of the central part of the disc is overdone, i.e. a bit too dark, in the author's calculation, a crude superposition of 9 single disc-on-straight-line illusion patterns, because the more spokes there are, the higher the average brightness value of the background for any single disc-on-straight-line illusion, and the lighter its illusionary grey bar.) 

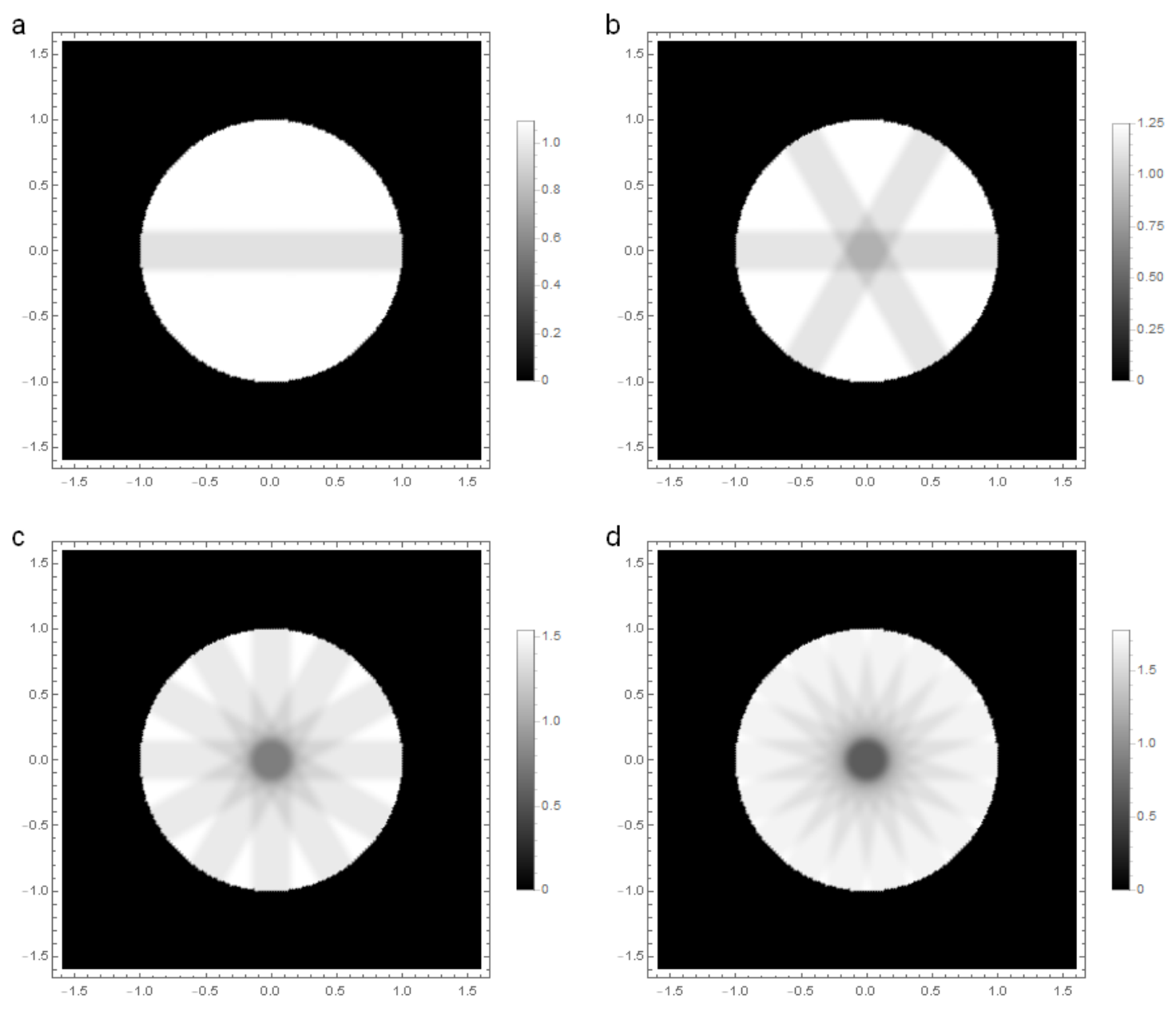

Figure 76

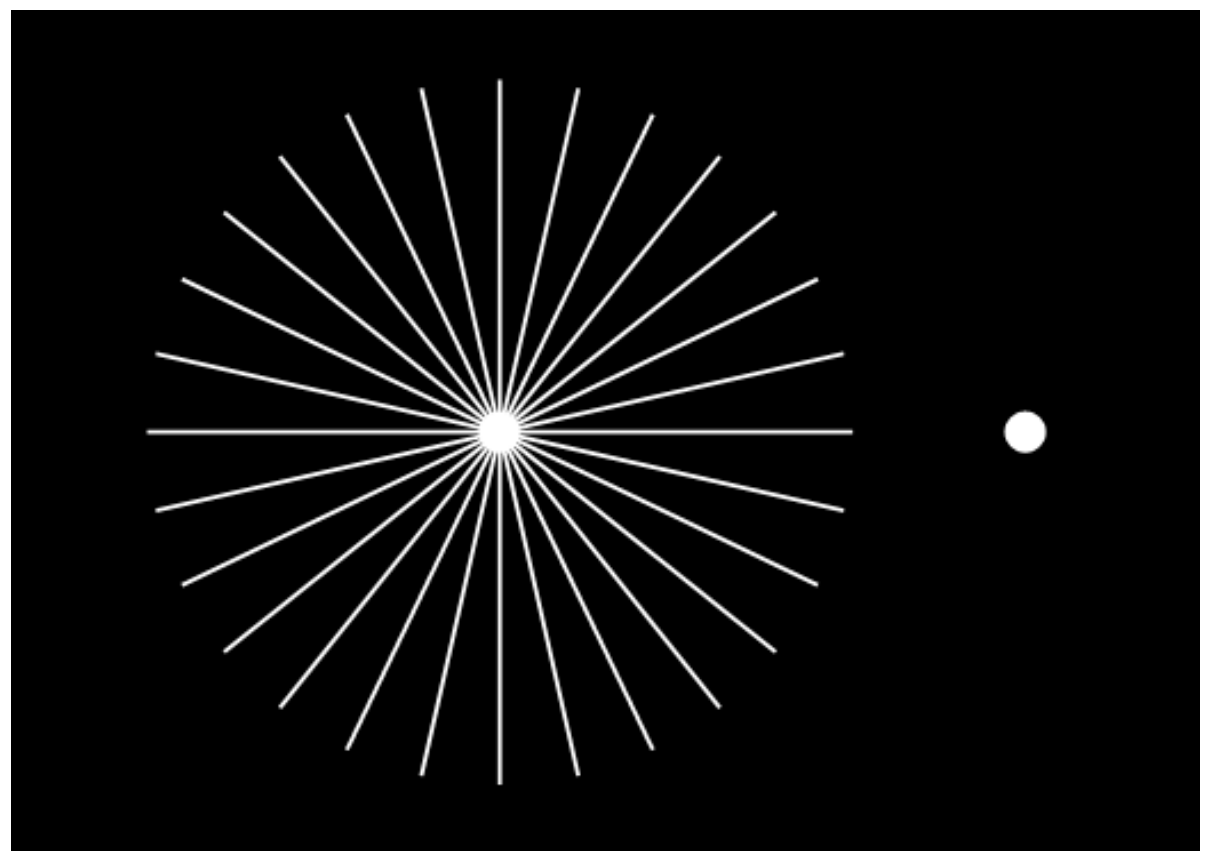

Figure 77 


\section{The Hermann grid and the scintillating grid illusions}

The less obvious illusion hidden in the Hermann grid having in a previous section been treated with isotropic filtering, now the commonly known Hermann grid illusion is about to be simulated below with anisotropic filtering. Clearly the Hermann grid pattern (Fig. 78) has two prominent directions, the horizontal and the vertical; there are therefore the horizontal and the vertical prominent flows of virtual particles in this pattern. Such recognition should be represented in the anisotropic filter to be used (Fig. 79c). The result of filtering the Hermann grid (Fig. 79a) with this filter is given in Fig. 79b. Incidentally, it can be seen in the filtering result that the grey blobs at passage intersections have each a roughly square shape, which, if one looks more attentively at the Hermann grid, seems to match one's visual illusion.

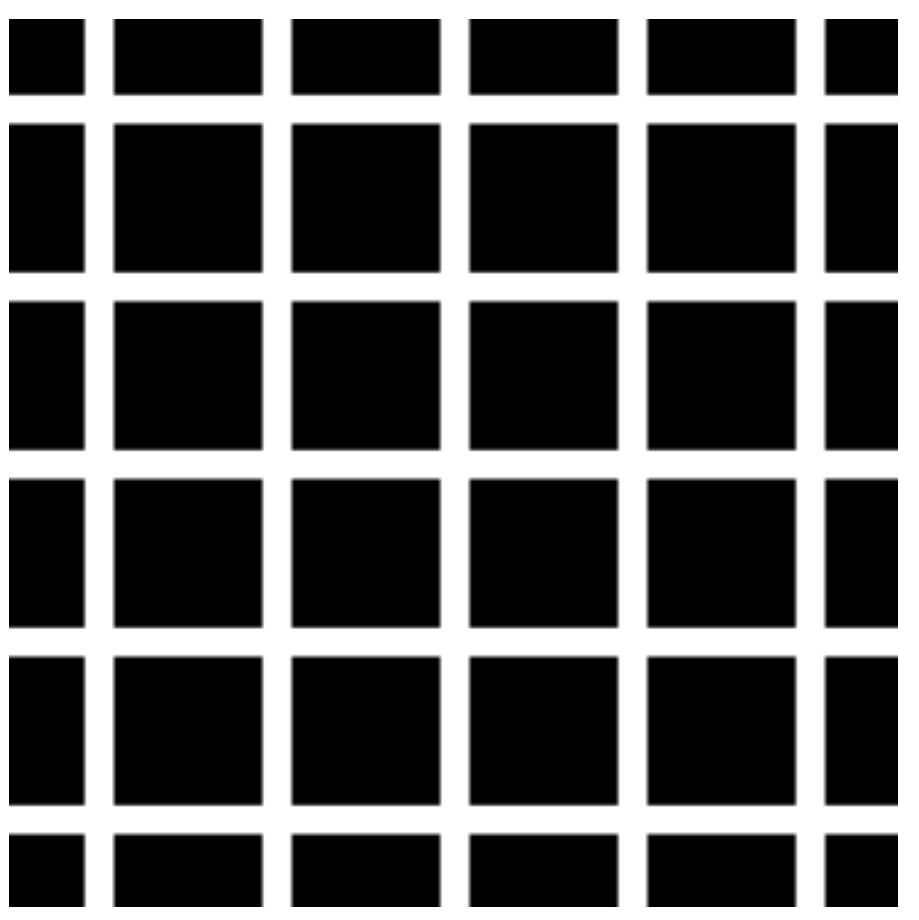

Figure 78

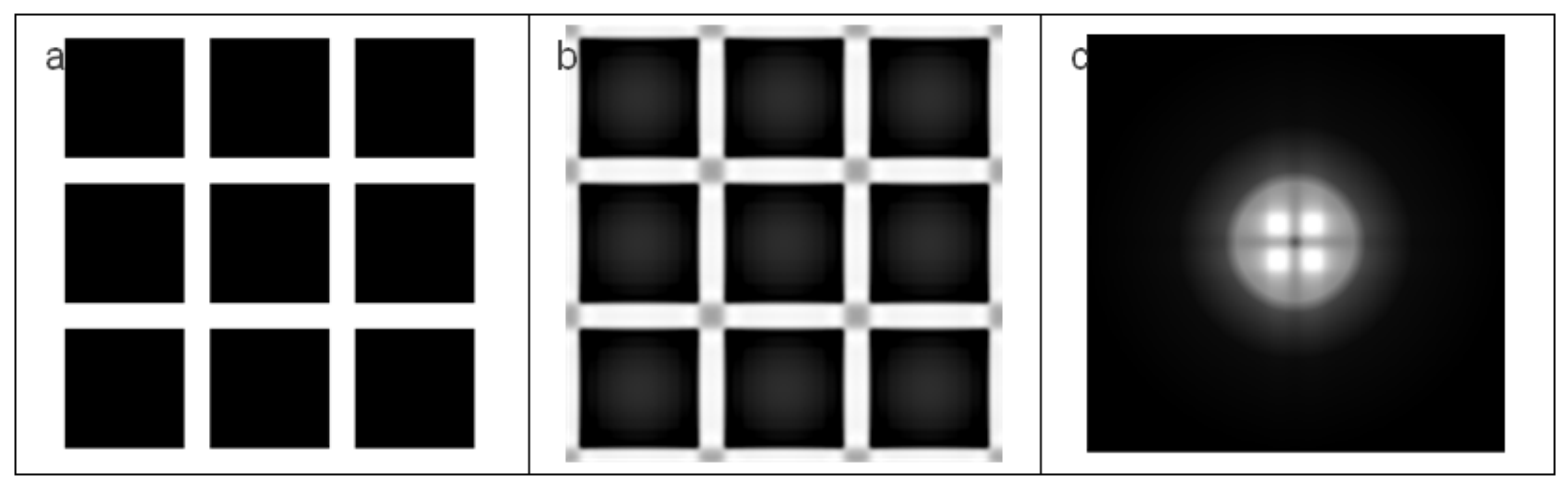

Figure 79 
When the white passages become wavy, however, as in Fig. 27, the prominent horizontal and vertical flows disappear, the visual filtering becomes isotropic, and what remains is only the much less obvious raster brightness illusion discussed earlier (Fig. 31).

Besides turning the white passages wavy, another way of destroying the Hermann grid illusion, this time able to affect a specific grid intersection, is as shown in Fig. 80, with two new oblique straight white lines going through the same intersection (here at the lower righthand part of the image). Owing to the addition of two paths of virtual particles, the intersection turns so much brighter than before that the original illusionary grey spot here vanishes, a phenomenon having been explained in the discussion of the spokes illusion.

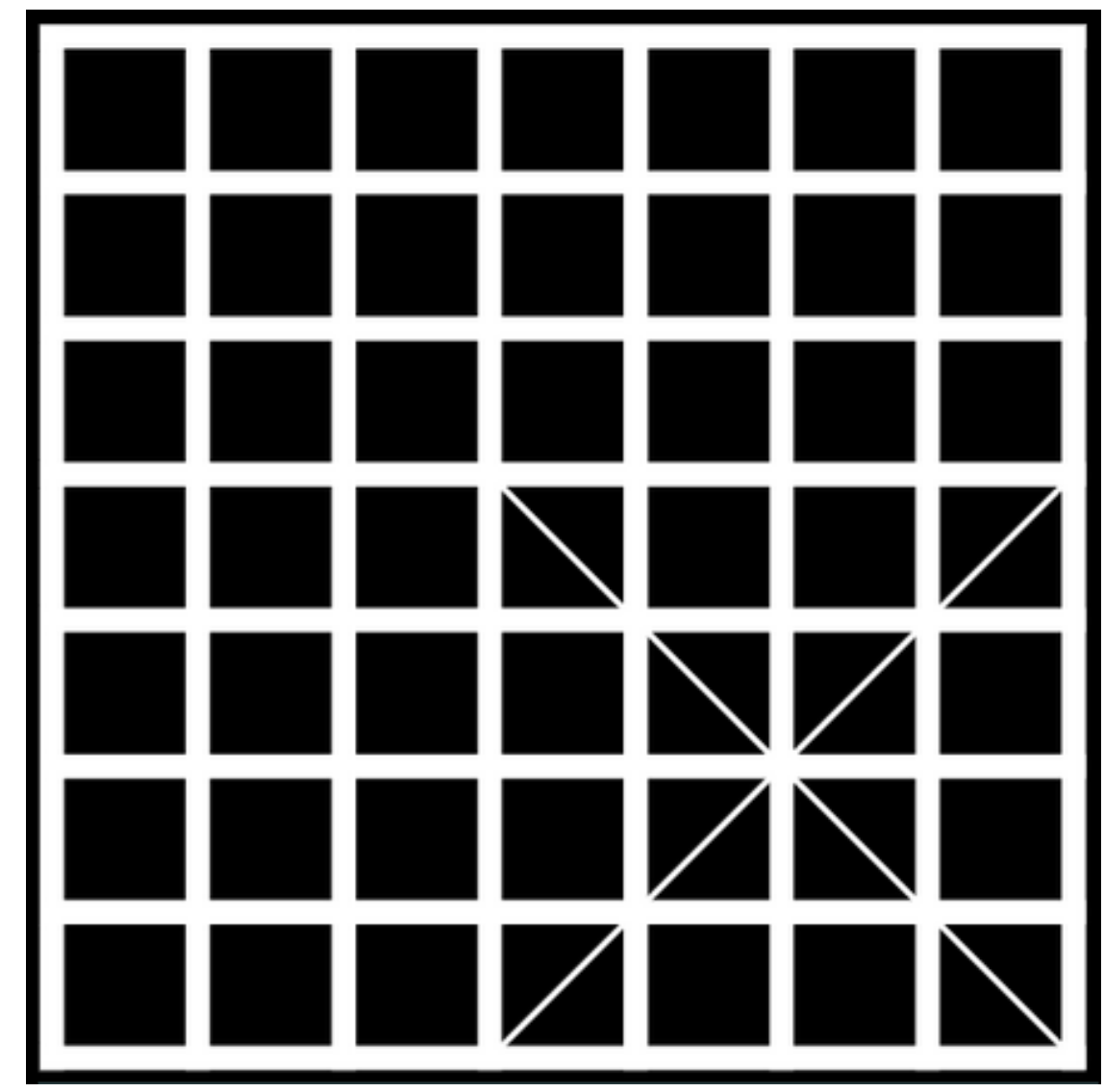

Figure 80 


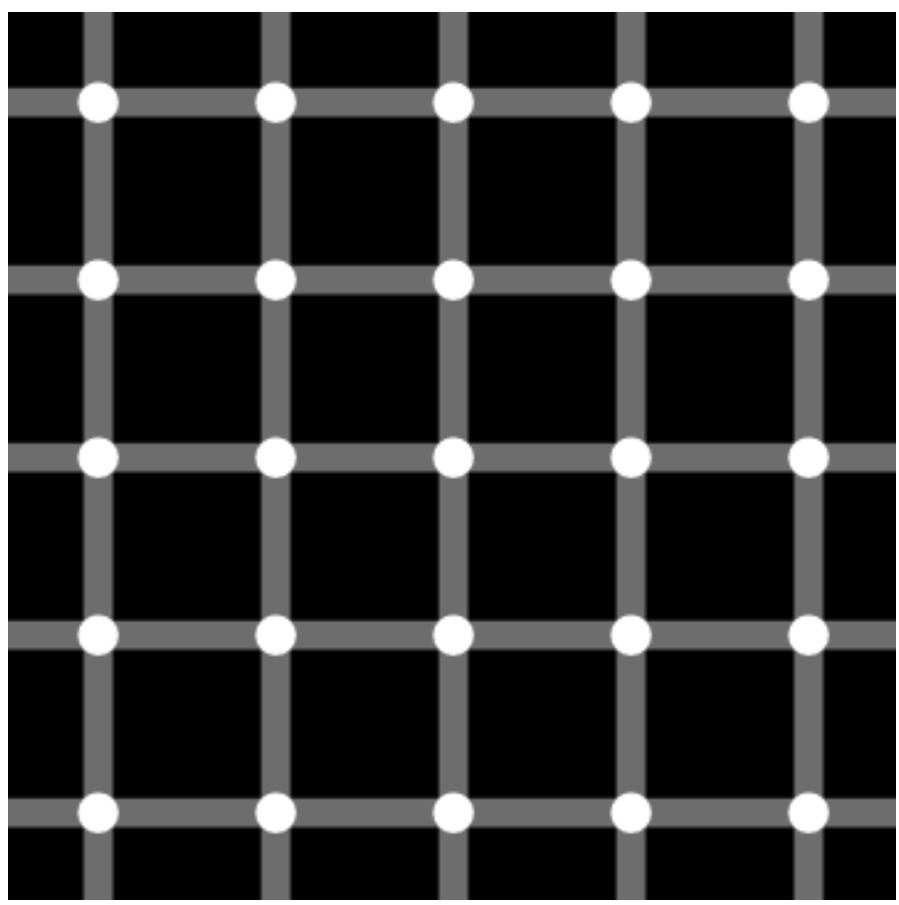

Figure 81

Closely related to the Hermann grid illusion is the scintillating grid illusion ${ }^{5}$ (Fig. 81): when one moves one's eyes around while looking at the figure, the white discs at the intersections flash or scintillate, with dark spots appearing inside the discs. The illusion is destroyed too when the grid lines become wavy. The basic nature of the scintillating grid is a darkened Hermann grid pattern (white passages having been changed into grey passages) all of whose intersections are covered by identical white discs of an appropriate size, large enough for a disc to fully cover an intersection, or more precisely the square-shaped overlap between two intersecting passages, but small enough so that the presence of the discs does not disrupt the horizontal and vertical flows of virtual particles in the Hermann grid pattern.

Analysing from the point of view of virtual moving particles, there are two strata of visual patterns: the stratum of the Hermann grid pattern, which is the primary one, and that of the isolated white discs at all the crossings. Thus grey illusionary spots are generated at the crossings in largely the same way as in the Hermann grid pattern, except that here the spots are darker because the grid is darker.

Now the mechanism of the scintillating grid illusion can be deduced. Firstly, there is in the visual system a blending superposition of those grey illusionary spots and the white discs, at which stage the scintillating effect is still absent. Secondly, when the observing eye moves around, a different mechanism, highly likely related to the visual system's sensitive detection of temporal changes in the visual field, breaks that blending and makes the contrast between the grey spot and its overlapping white disc briefly but hugely sharper, hence the scintillation.

To conclude this section, a colour figure is added (Fig. 82) to illustrate where the grey spots of the Hermann grid illusion acquire their 'greyness' from. The background of the left half of Fig. 82 is red, and it is interesting to see that the grey spots at the white passage intersections of this left part are, correspondingly, reddish; likewise, the background of the right half of the figure is blue, and the grey spots of this right part bluish. The main source, therefore, of the greyness of the grey spots in the Hermann grid illusion is the background of the white 
passages. (The same reasoning and conclusion apply also to the raster brightness illusion in the Hermann grid, discussed in a much earlier section.)

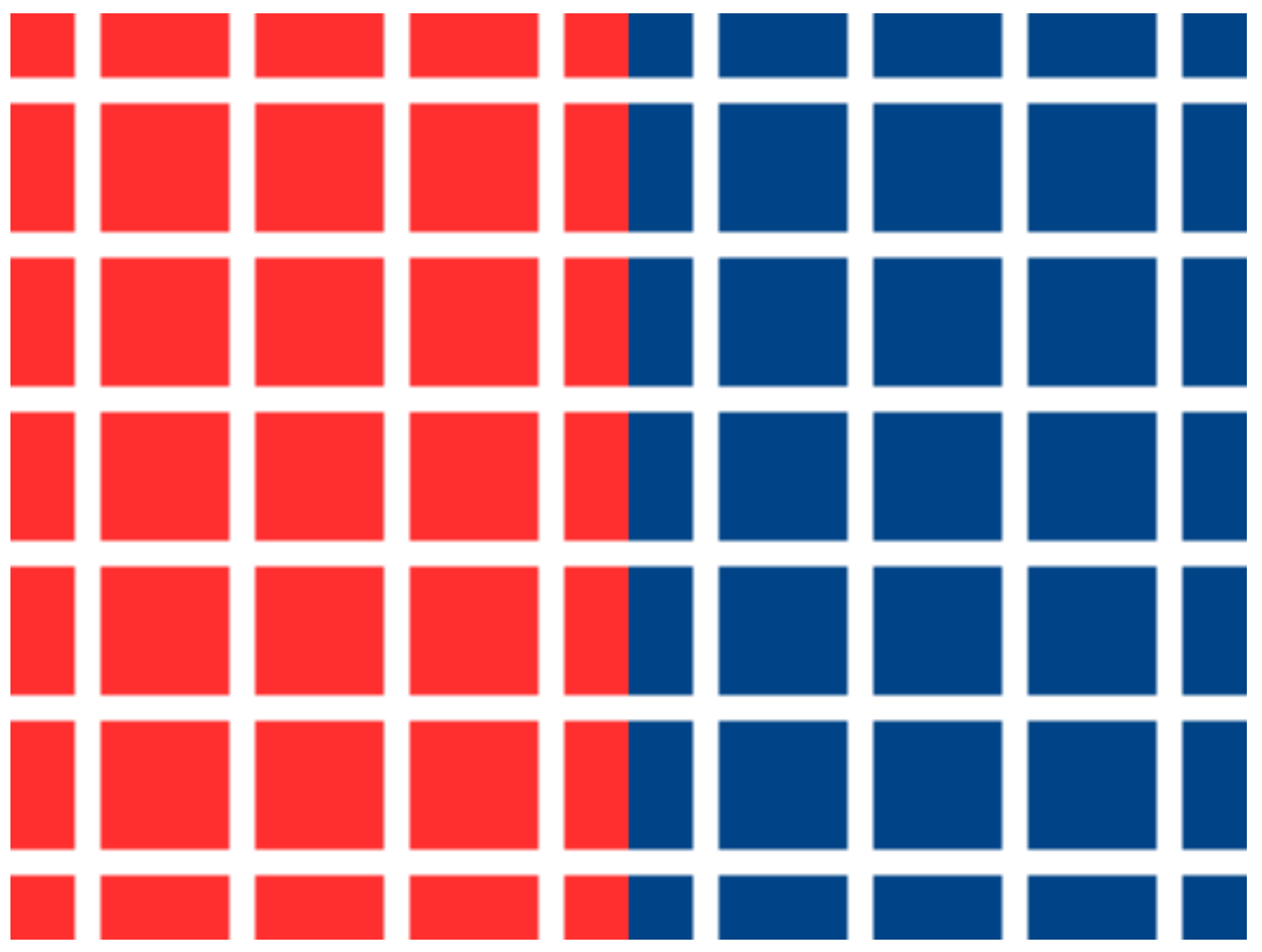

Figure 82

\section{A short retrospect}

Fig. 83a, -b, -c, -d, -e, and -f are a series of spokes-illusion figures with an increasing number of spokes. In that order, they represent a gradual transition of illusions that are intrinsically similar, from the vague horizontal grey bar of the disc-on-straight-line illusion (Fig. a), through the vague dark dot on the white central disc of sparse spokes (Fig. b and c, with the former being in a sense a variant form of the Hermann grid illusion), and through the increasingly bright central discs (Fig. $\mathrm{d}$ and e), to the shining bright central disc (Fig. f). 


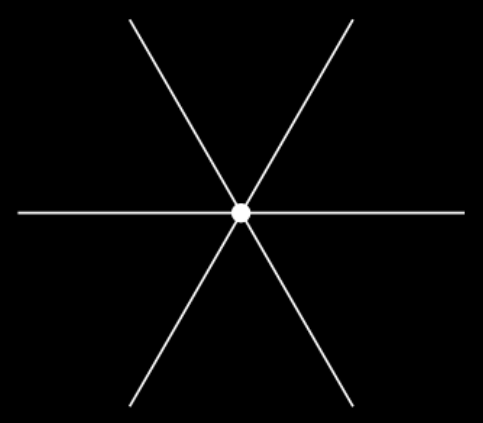

e

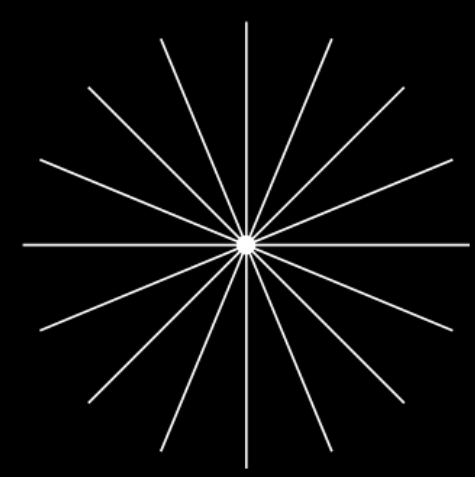

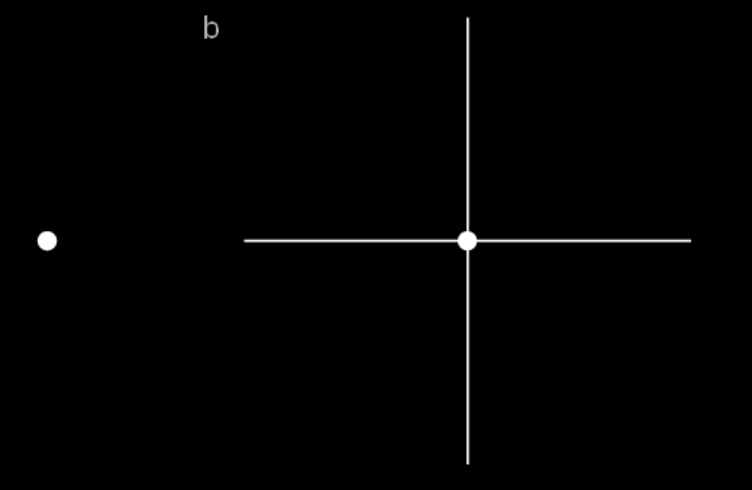

d

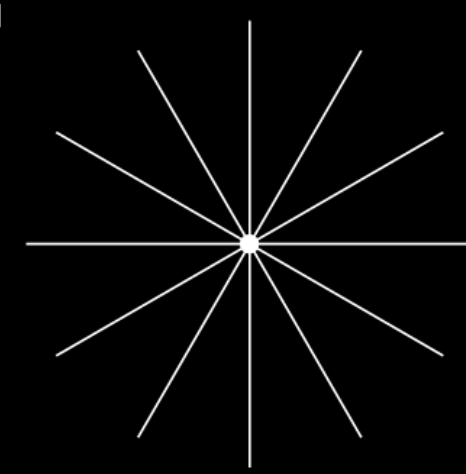

f

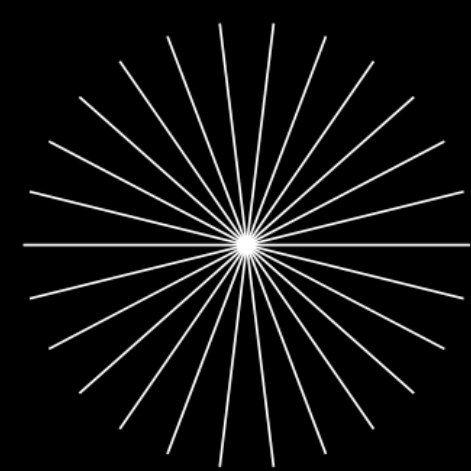

Figure 83

Now that the many vector brightness illusions discussed have been seen to be all caused by certain straight lines, the impression may have been created that the word vector in this paper means 'straight' or 'of a straight line'; but that is false, for which the author is to blame. Instead, here it means 'having direction and sensitive to the given shape and geometry'. Correspondingly, by raster is meant the opposite. Thus those geometrical illusions treated earlier can also be called vector illusions.

Further, a general comment on all the visual filtering models given in this paper is now appropriate. It is plain that visual perception is first the work of millions of receptor cells and other neurons in the eye and then the combined activity of millions of neurons in the brain. Thus it is safe or at least reasonable to assume that any visual filtering model having been used, e.g. the anisotropic filtering in vector brightness illusions, is a statistical outcome of millions of more basic and smaller-scale activities, and probably capable of being modelled 
by a field of virtual moving particles at a deeper level. It may be analogous to this, familiar in the subject of thermodynamics: the pressure exerted on a wall of a container by the gas it contains is caused by billions of billions of the gas molecules colliding, in all possible sorts of ways, with that wall; what is more, the macroscopic pressure can be statistically calculated from the microscopic collisions.

\section{Size illusions}

Similar shapes of distinctly different sizes, when placed closely to each other, will appear to the human visual system in such a way that their difference in size is amplified; this is also explicable by the generalized Gibbs phenomenon. In the Ebbinghaus illusion (Fig. 84), for example, the inner circle to the left, surrounded by tiny circles, looks larger than the inner circle on the right, surrounded by large circles. In truth these two inner ones are identical in size.
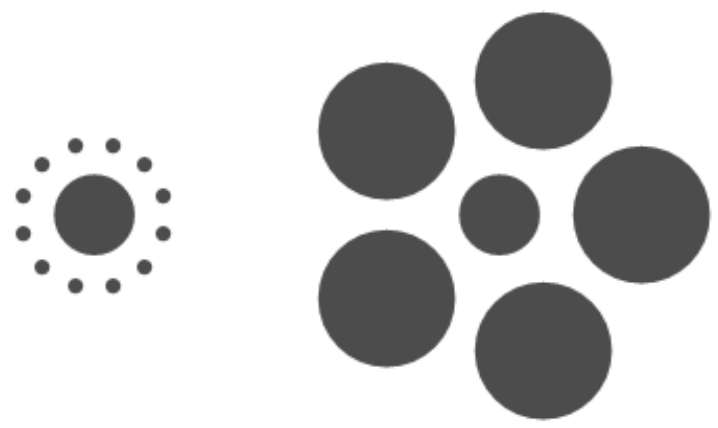

Figure 84

Detailed mathematical simulation of this effect is not covered in this paper; it would presumably be more complicated than is the case with those illusions discussed above. As a whole it would in certain ways be akin to the treatment of multi-path figures in which the lines do not meet (Fig. 58a); hence essential would be a size difference amplification factor as a function of distance between different shapes. The distance between two shapes, despite sounding simple and unambiguous, may pose problems because each shape comprises an infinite number of points, whereas a distance is between two points only; as a possible solution, the centre of mass of a shape can be adopted as representing the position of the whole shape.

\section{Motion illusions}

When one is looking at moving objects, it is likely that how they are seen moving is not how they actually are moving. The human visual system creates arguably no weaker illusions with dynamic objects than with static ones. 
Application of visual filtering to the motion (also two-dimensional) of a single, visually observable particle, or rather, a dot, is straightforward. First, parametric equations of the dot's trajectory should be given, namely, its position $(x, y)$ as a function of time:

$x=x(t) ; y=y(t)$.

Then the trajectory is discretized into a great number of sample points, with equal and very short time intervals between consecutive ones. Next, these sample points undergo visual filtering, to generate the illusionary dot motion.

That is the same method used in the case of single-path figures discussed earlier. In the case of multiple particles moving simultaneously, the method dealing with multi-path figures should be transplanted here. To this a separate section will be devoted.

\section{The step jump motion}

As indicated in the form of a path in Fig. 85a, the particle, or dot, first moves from left to right at a constant speed along the lower horizontal line; then suddenly it jumps vertically up and immediately moves at the same speed and in the same direction along an upper horizontal line, its path resembling a step. Ideally the vertical jump is instant and takes no time at all. To be precise the vertical part of the step path represents, kinematically, a trajectory segment with infinite speed along it, or, mathematically, a discontinuity of a function; this is just what is needed. To the human viewer, an evident generalized Gibbs phenomenon can be observed at the step jump, as shown by the black curve in Fig. 85b, which is the result of filtering the step path in Fig. 85a with the filter shown in Fig.85c, and behind which the grey line is the original step path. One should of course look directly at a movie or an animated image of this motion, not at the static figure given here.

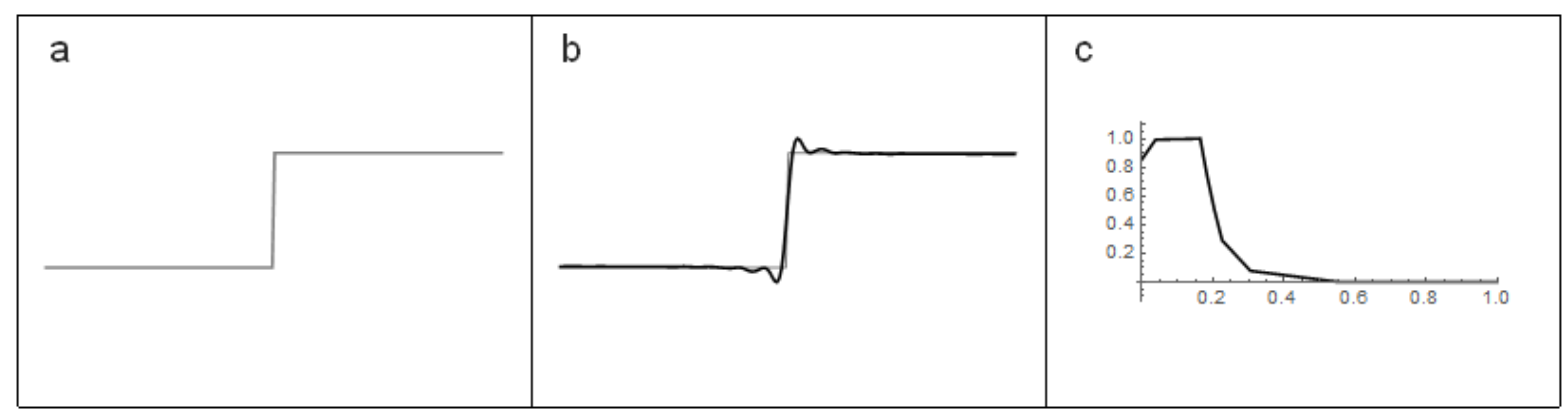

Figure 85

\section{Regular polygonal motions}

A dot moves at a constant speed along the straight sides of a regular $n$-sided convex polygon (Fig. 86); $n$ is here chosen to be 4,6, and 10, corresponding to the three polygons, from left to right, in the figure. Fig. 87 gives the paths (the black curves) of the particles after visual filtering, on top of the original polygons (the grey lines). It is extremely obvious that the motions as seen by a human viewer are strikingly similar to those depicted in Fig. 87 (in this 
paper, only their paths can be shown). Here we see again the shrinkage of single-path angles as in Fig. 45, and once more the illusory motions are examples of the contiguity illusion, or equivalently the generalized Gibbs phenomenon.

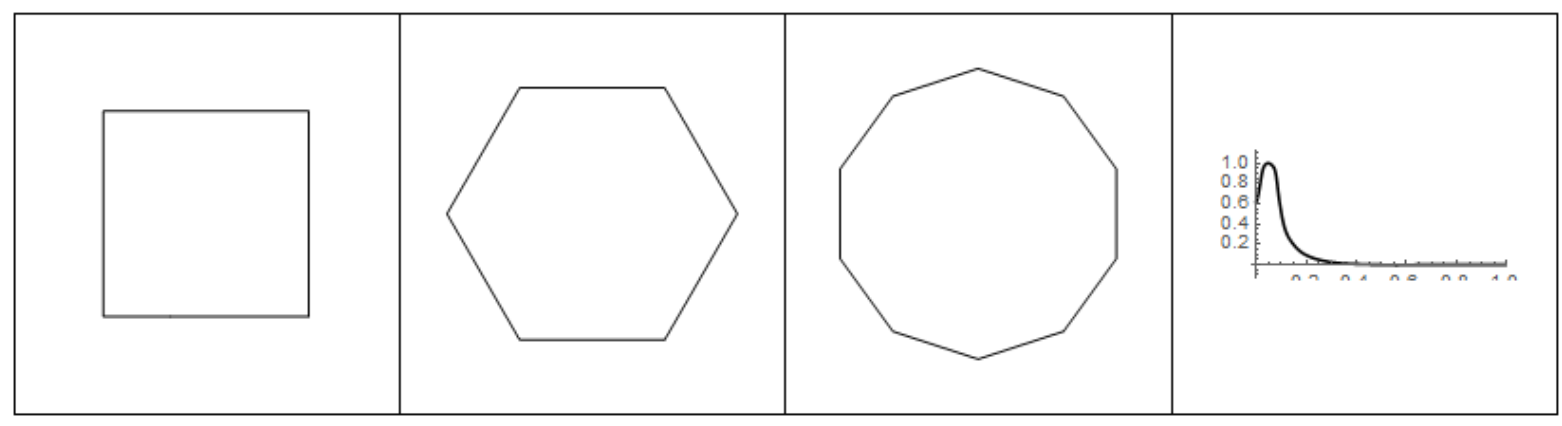

Figure 86

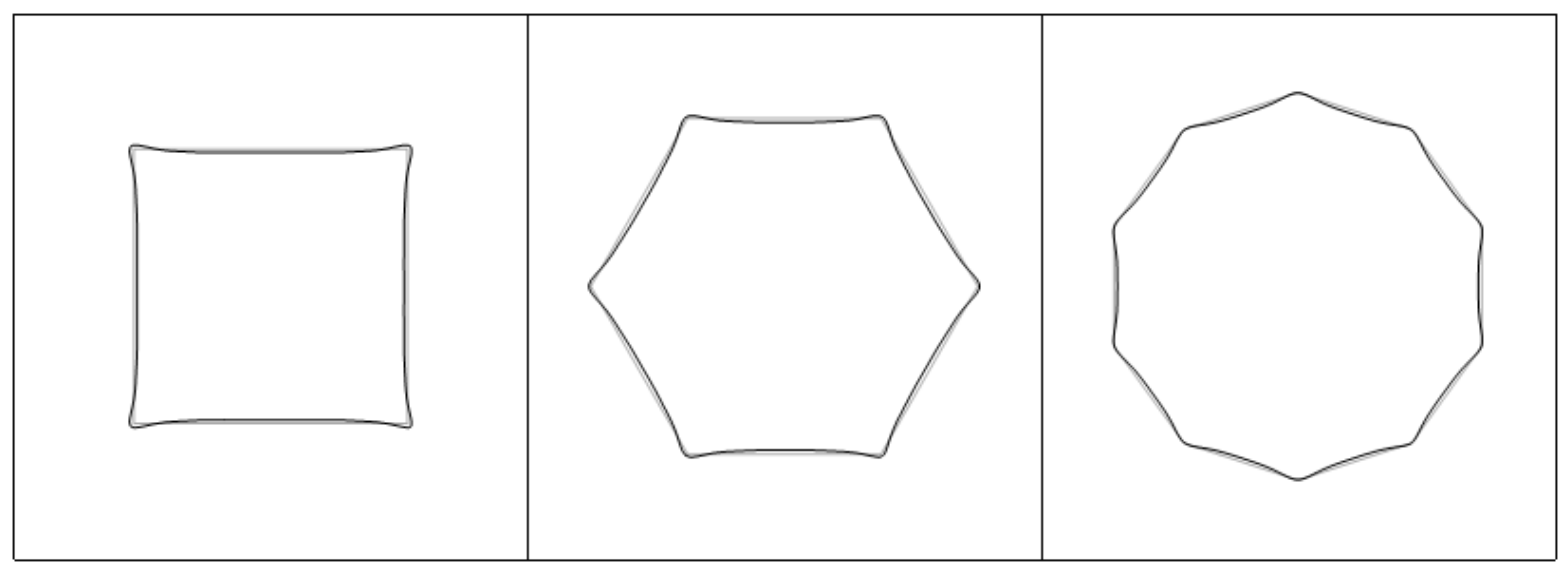

Figure 87

The filtered curves are aesthetically more attractive than the corresponding original regular polygons, especially from the point of view of Chinese calligraphy; but such a conclusion may not appear apparent to people without experience in this art. In any case, a bit of imagination is perhaps worth taking. One can imagine, for example, that the two 10-sided polygons in Fig. 86 and Fig. 87, original and filtered, respectively, are the cross sections of two huge stone columns that are otherwise identical. Which of the two columns is the more beautiful? At least the ancient Greeks would have chosen the latter.

\section{Multi-path motion illusions}

The two examples above of motion illusions are both single-path, namely, involving only one virtual particle and no interactions between different particles. However, such interaction occurs in multi-path motion illusions. Experiments on multi-path ones can readily be conducted based on relevant methods discussed in the sections Angle expansion of intersecting lines and Illusion of a pure multi-path figure, and have been done, with the result that the illusions are very well modelled.

For example, the motions represented in Fig. 53b, of two dots (but, in that section, of two virtual particles) starting simultaneously from $\mathrm{A}$ and $\mathrm{C}$ respectively and moving to the left at 
the same speed along the two straight lines (which themselves should be absent in the animation), generate in the visual system illusionary motions whose paths are as simulated in Fig. 55. A noteworthy trait of the simulated paths, not yet mentioned, is the transverse shift of each of the four path segments that makes the in-angle $<$ AOC and out-angle $<$ DOB swell. The faster the two dots move, the more observable the swelling, namely the shifting of their illusionary paths. When the two paths are perpendicular, in particular, the illusionary shifting of the paths is perhaps the largest.

In addition, what is easier with motion illusions is that the motions of dots are specified freely by the experimenter. The multi-path geometric illusion construct of Fig. 58a serves as another example; it can be turned into a multi-path motion illusion animation in which a white dot moves anticlockwise at a constant speed along the left half of the circle (without the circle's presence, of course), and another white dot moves at a constant speed downward along the vertical straight line (without the straight line's presence), the two white dots being so synchronized that at a certain moment they reach the midpoints of their respective paths simultaneously, thus getting as near to each other as allowed by the geometry in Fig. 58a.

\section{The turning strokes in Chinese characters}

A great many, or perhaps most, Chinese characters have one or more turning strokes. Such a stroke comprises two straight, or relatively straight, segments, normally of different lengths, that are written consecutively as one stroke, and together form, approximately, a right angle, or in certain cases an acute one. For instance, immediately below the next paragraph is the same Chinese character as displayed in two different fonts, and at its upper right corner is a right angle formed by such a turning stroke.

In the stylistic variation of Chinese calligraphy considered as the most basic and the most formal, namely that which is called 楷书 (Kai Shu), the turning stroke very often has, where the bending occurs, a gentle protrusion, somewhat similar to the protrusions occurring in Fig. 45 and Fig. 87. As an illustration, the first large character below this paragraph, which is in a style of Kai Shu digitized as a computer font, has such a protrusion at the upper right corner of it, and another one inside the character. (Strictly speaking, the protrusion at a stroke corner is often only one of the two things that the calligrapher does there at the same time, the other being a small pressing manipulation of the brush that effects at the corner what in a way resembles a chamfer. Because of this additional slightly chamfer-like segment, there are now two obtuse corners close together, and each of them may have a very small protrusion.) In fact, this phenomenon is most distinctly seen in the traditional and predominant typeface of Chinese characters, 宋体 (Song Ti), having been in use for hundreds of years; the second large character below is an example. Additionally, although the two sides of the upper left corner of this character belong to two separate strokes, in the overall appearance of the character that is also a right-angled corner with an obvious protrusion.

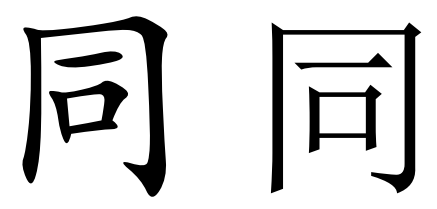


Apparent counterexamples, however, also exist, wherein there is no protrusion at the turning point. For instance, in certain styles of Kai Shu by masters the presence of the protrusion and its absence are equally noticeable, and it is completely absent in any variant of the typeface called 黑体 (Hei Ti), which is in a certain sense analogous to a sans serif typeface of the letters in English. Below is that same character as displayed in $\mathrm{Hei} \mathrm{Ti}$. Nevertheless, it can be argued, and is really apparent at least to some, such as the author, that the one below is, so far as the shape of the stroke's bending is concerned, somewhat less attractive than the two above, owing to the lack of any details specially designed to accommodate the abrupt turning transition between the two segments in the stroke. Those specially designed details no doubt have, among others, a decorative function. A turning stroke with no decorative protrusion is basically aesthetically neutral, whereas that with a small, well-designed protrusion is aesthetically positive.

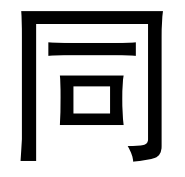

As a matter of fact, in the predecessor of Kai Shu, namely 隶书 ( $\mathrm{LiShu}$ ), or to be precise the ancient $L i S h u$, such protrusions in bending strokes are much less often found. In this sense, we see an evolution in Chinese calligraphy from the 'bare' corner to the more sophisticated corner with protrusion. (Note that here only the corner of the turning stoke is being discussed, and that that evolution by no means suggests that $L i S h u$, as a variation of calligraphy, is aesthetically inferior to Kai Shu.) With respect to the level of complexity of the corner of a turning stroke, $\mathrm{Hei} \mathrm{Ti}$ (e.g. the character immediately above) is comparable to $\mathrm{Li}$ Shu; and Song Ti, on the other hand, to Kai Shu.

(A closer inspection of the character above in $\mathrm{Hei} \mathrm{Ti}$, best performed by greatly enlarging the font, reveals, unexpectedly, that its turning strokes have been designed such that their corners, unlike other styles of $\mathrm{Hei} \mathrm{Ti}$, have very slight protrusions too, certainly in order for these strokes to look more agreeable.)

There are two main reasons for which a protrusion at the turning point does not always seem to be that necessary, and neither of them really contradicts the virtual particle theory on lines.

Here is the first one. If the abrupt turning is so modified as to become a small, slightly chamfer-like stroke, or even an arc, the need for a protrusion here is diminished, according to the simulated deformation of single-path figures. An illustration of that is the bulb illusion (Fig. 41), where the swelling is tiny, far from being comparable in magnitude with those in Fig. 45 and Fig. 87. This may explain the absence of the protrusions at the turning points in certain styles of Kai Shu, and in certain other variations of Chinese calligraphy.

The second reason is this. The protrusion as found in Fig. 45 and Fig. 87 arises manifestly only when the angle in question is a single-path figure (a topic discussed in the section Deformation of single-path angles). If the multi-path aspect is also taken into consideration, the illusion of angle sharpening and protrusion will be considerably decreased for both the right angle and the acute angle, so that no illusory protrusion can normally be observed with ease when the angle is viewed in isolation. In this connection, the presence of a protrusion at the corner of a turning stroke and the absence of it there are no longer contradictory to each 
other, but merely two alternatives for the calligrapher to choose between in harmony with his or her personal style.

The following character, as in different fonts, is another example for this section.

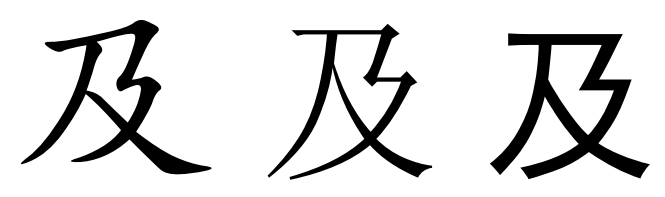

Whether or not the facts and analyses in this section can be regarded as evidence that supports the theory having been proposed on geometrical and motion illusions, the author is not yet sure, because counterexamples can often be found, and the dispelling of them that has been given does not now seem to be perfectly conclusive. In spite of that, an inconclusive discussion can still be worthwhile, for showing theoretical possibilities of which further explorations of the subject may obtain either confirmation or refutation.

\section{Aesthetic elevation of the colours of an image}

In Fig. 88 (where sub-labels, $a$ and $b$, for the two images, have been left out, in order not to interfere with the effects intended in relation to fine art), the left image is a computer generated 3-D graphic designed deliberately to be rather unsatisfactory in terms of colours, at least to the eye of a painter in oil. The red colour of the red cube (there being, to be precise, different shades of red), in particular, is unpleasant for being too strong and harsh, and lacking in harmony with its environment, mainly the bluish grey background; the large size of that red contributes significantly to its harshness too. It is almost the same with the brown colour of the brown cube, but to a lesser extent.

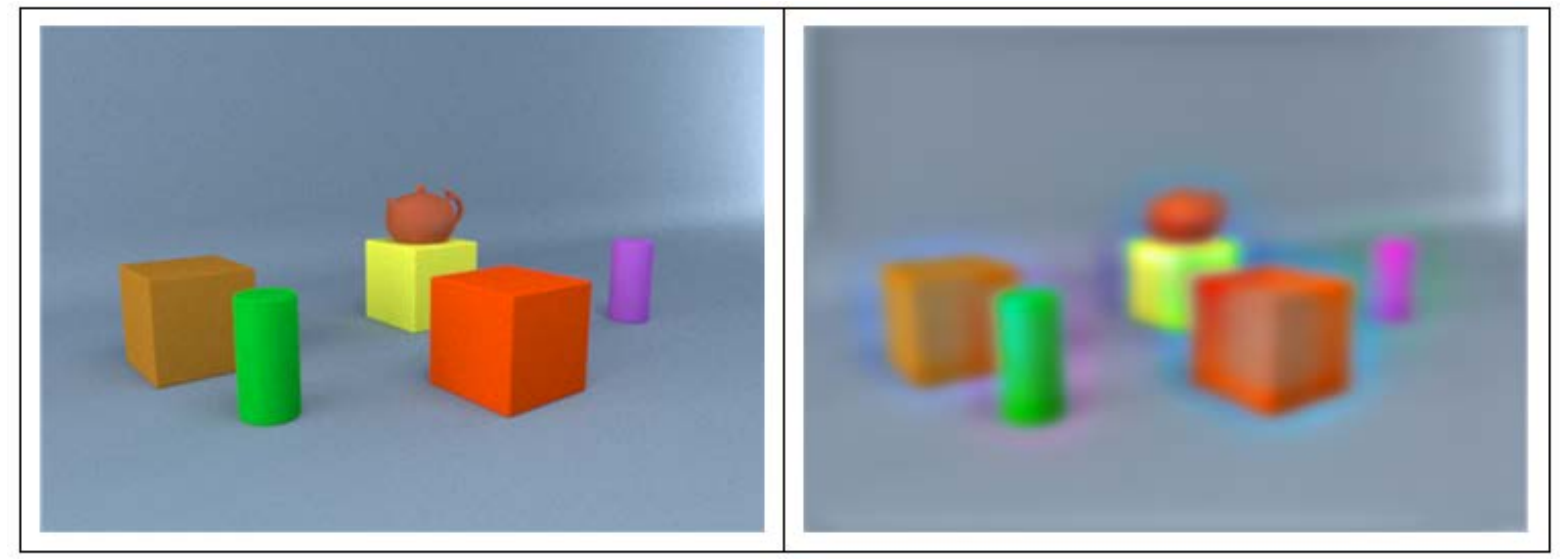

Figure 88

The right image of Fig. 88 is the visual-filtered outcome of the left one. Particularly noticeable in this filtered version is the appearance of bluish grey colours on the red cube, due to the large areas of bluish grey of the background being entangled in the filtering process with the red colours of the red cube. Harmony thus arises between the red cube and its environment. The same is true of the brown cube, which now looks more mellow and 
agreeable. Meanwhile, around the red cube in the filtered image, a gentle, light blue aura has been created, adding, along with the grey colour within the cube, to the vibrancy of the colour pattern of the image.

The overall effect of the Fourier visual filtering is that the pattern of colours of the picture becomes evidently more harmonious and more colourful simultaneously, i.e. a marked increase in unity and variety. The reason, as treated earlier, is that the visual filtering, besides enhancing colour contrast between adjacent colours, makes colours in different parts, in fact all parts, of the image be more 'entangled' with each other, such entanglement being a crucial part of the beauty of the art of oil painting. In a word, the Fourier visual filtering has made the image more painterly and cordial to the human eye.

The non-expert in oil painting would perhaps find it unacceptable for the red cube in the filtered image to bear those obvious grey parts on it and that blue aura around it, thinking that with those greys it is no longer the original red cube anymore, an astonishing breach of reality. If hearing such thoughts being expressed, the painter would answer that rigid, exact depiction of the external world as it is - from an aesthetic rather than functional point of view - is never the goal of true art, which seeks instead aesthetic elevation of the objective world, and that the filtered image is, to the trained eye, indeed aesthetically superior. As will be illustrated in a section below, those unrealistic colour patterns existing in the filtered image are fairly common to works by masters. Nevertheless, even to some painters the effects of the Fourier visual filtering in Fig. 88 (right image) may appear somewhat overdone; but the strength of that filtering can of course be reduced, so that the output image looks pleasant to all artists, and to non-experts as well.

Actually the strength of filtering is probably not the main reason for which the filtered image may appear somewhat overdone to painters. That reason appears to be this: following the colour illusion described by Fourier visual filtering, there often is a secondary illusion taking effect that resembles, or perhaps is, the watercolour illusion, as was previously mentioned in the section The Gibbs phenomenon as a good thing (more specifically, the paragraph beginning with 'yet'). What it does is, for example, to 'dissolve' into the whole red cube shape the bluish grey that is now seen only in the central area of the shape; the final result is a red cube which as a whole is a little greyer, and thus mellower, than before, but which does not have an obvious central grey part as in the filtered image. This painters often do.

Roughly in opposition to the above method of spreading and weakening of the illusionary colour, there are also other things they often do which make the effects of Fourier visual filtering exemplified in Fig. 88 rather subtle and fairly difficult for the non-expert to notice. The grey area on the red cube, for instance, produced by the filtering, may be seen on a painting as some grey strokes intermingled cohesively with red strokes that depict the red cube. Alternatively, the grey area may be rendered considerably smaller (perhaps as the colour of certain little objects) than in the filtered image.

In almost all cases, it is not hard to find examples from works of art, especially paintings, by masters. Some selected illustrations will be given below, but before that is a section about the mind's eye of the qualified painter, which is first mentioned in the section The simultaneous contrast illusion. 


\section{The mind's eye}

The existence of a surprising phenomenon called blindsight is known to psychologists: some blindsight patients, in particular, can identify an object's colour, while insisting that they do not see the colour but are just guessing. The mind's eye for colour illusions in the qualified artist can be thought of as a form of extra blindsight, for colours, existing in a normal, healthy visual perception system - a function which the normal, healthy visual perception systems of other people do not possess. The artist, during years of being an apprentice or student in a studio, acquires the mind's eye as here described by learning to draw and paint in the correct ways and, no less importantly, by making efforts in studying excellent works of art, especially those by masters; also, a gift for it will definitely be of substantial help. This extra blindsight, the mind's eye, creates rather vague brightness and colour illusions that people without it do not quite understand. Further, in the case of qualified artists, it is more likely that there are continuous intermediate states between the capacity for seeing such illusions completely consciously and that for seeing them in a blindsight fashion.

\section{Analysis of some works by Western masters}

The range of painters in oils from whose works illustrative examples can be chosen is certainly not limited to the very small set of highly acclaimed masters. Masters, however, are the most convincing, who are regularly discussed in books on art history and whose works are easy to find in art museums in the West. Hence the selection is from works by masters.

In the collage White Seaweed by the 20th-century French painter Henry Matisse, of which Artwork Fig. 2 (figures of works of art are included at the end of this paper) is a reproduction, the red areas in the middle of the white leaves and the stem are obviously analogous to the bluish grey areas on the two cubes in the filtered image of Fig. 88. Here, it is worth noting, the artist was looking not at real seaweed but at his white plant in paper against its red background (Artwork Fig. 3) when he sensed the need for adding the central red pieces of paper within the white plant. In other similar seaweed collages of his, it may be noticed that Matisse did not choose the background colour for the patches in the middle of the leaves, which seems to be a contradiction to the current example. There are two points to know about this seeming contradiction: $(a)$ if the drawing aspects of the artworks are considered (or, simplistically, when they, despite being in colour, are seen as being black and white), there is no contradiction but only agreement; $(b)$ depiction of the type of visual illusion discussed here is not the sole aim of fine art, and other considerations (such as trying to achieve vibrancy in colour) are functioning too that can lead the artist to avoid choosing the background colour for the illusionary grey or dark grey central areas.

From the above example we see that the visual illusions do not come only from observing the real world objects being painted, but they also emerge from observing the work itself in progress; moreover, the less realistic the painting, the more the illusions come from contemplating the artwork in progress.

Reproduced in Artwork Fig. 4, 5, and 6 are three oil paintings of children by the French Impressionist painter Auguste Renoir: Child in a White Dress, Child with a Whip, and Child with a Doll, respectively. In the first of these works, the blue background of the child's face generates at the centre of the face a blue illusionary area, which is then concentrated by the 
painter as the blue colour of the child's eyes, as discussed several paragraphs back. Now there seems to be room for suspicion that it is pure coincidence that the blue colour of the eyes matches the colour of the background; there will be none, however, if more paintings are investigated. In the second of these three paintings, the background of the child's face is primarily brown, those other background colours being much farther away from the face; consequently the child's eyes are brown. In the third painting, the colour of the background of the face is primarily dark grey interspersed with many black strokes; so the eyes are black.

Renoir was not the only master that did this with the colour of the eyes being rendered, and it also happened to some others later than the Impressionists, painters in oil earlier than that being more concerned with the representation of physical reality and much less free in searching for and expressing what they could see beyond that reality with the eye and the mind's eye. Artwork Fig. 7 is a reproduction of Portrait of Joseph Roulin, a further example, by the Post-Impressionist Vincent van Gogh. Here we see a totally-green-eyed and blueeyebrowed man, whose face, especially the nose, bears some green strokes too. His background is green and his uniform blue. The relevant analysis can be omitted, but reference should perhaps be made to a slightly earlier paragraph, beginning with 'roughly'.

It may be suspected, in the case of the portrait just mentioned, that some green light reflected by the green background (possibly a large piece of green cloth hanging behind the model) reached the man's face and thus turned it, especially the eyes, greenish, so that the painter was actually depicting only the green colour that was physically present, instead of his colour illusion. This idea is wrong, because the amount of green light that was finally able to reach the man's face was too weak to produce the strength of the green colours applied to the man's nose and, above all, to his eyes. As a fact that should be taken into consideration, painters do not have, in front of or around the model, other large pieces of cloth the same colour as the cloth behind the model. So the green diffuse light reflected from the green cloth in the background, already very weak, had first to reach some other objects (for example, a wall far away in front of the model), and then to be diffusely reflected a second time, before reaching the man's face, from which it had to be diffusely reflected a third time to reach the painter's eyes. It was absolutely impossible, therefore, for the man's eyes to physically appear as green as in the painting; and hardly possible for other parts of his face to be physically as greenish as the corresponding strokes on it in the painting. Thus, by an analysis that can be applied in general, the sort of doubt as expressed in the first sentence of this paragraph is dispelled.

Likewise, a work by the Post-Impressionist painter Paul Cézanne can be added, who had profound influence on Western painting after the turn of the 20th century. Artwork Fig. 8 is a reproduction of his painting Boy in a Red Waistcoat, where relevant to discussions in this section are the two or three green strokes in the middle of the boy's left hand, hanging down at the bottom of the painting. The hand is mainly of different tones of brownish red, but it is surrounded in effect by a large amount of green, i.e. a greenish background (in it there are of course bluish and yellowish areas, but the combination of blue and yellow is green), hence the colour illusion of a green area in the middle of the hand, similar to the bluish grey in the middle of the red or brown cube in the filtered image of Fig. 88. In this case, as in all previous works of art mentioned in this section, the strokes are an 'echo in colour' from the background. 
It will be interesting to observe further that in the painting Girl with Racket and Shuttlecock by Chardin (of which Artwork Fig. 1 is a colour reproduction), talked about in a much earlier section, there are some objects hanging from the girl's waist, seen right in the middle of her white skirt, and that these objects, though of different colours, are also required to appear by the eye or the mind's eye as in the previous cases.

Perhaps a little surprisingly, it was not very rare for Cézanne to paint auras around people or objects, like those found in the filtered image of Fig. 88, though less regular in shape and in colour. In his Woman with a Coffee Pot (reproduced in Artwork Fig. 9), blue auras are seen around the woman's head, especially around her hair and beside the part of her face in darkness; also, a brownish or reddish aura can be found touching her left shoulder, which is in shadow. If, in addition, a 'dark aura' can be called an aura as well, we can find such things touching the woman's right cheek (the left part of the face in the painting), right shoulder, and right arm. Further, around the cup, saucer, and spoon, which are on the table, auras also appear. Incidentally, there is an area of different shades of blue in the middle of the woman's left hand, in yellow, whose background is the blue skirt; as with the boy's left hand in Artwork Fig. 8, an equivalent occurs again to the bluish grey on the red cube in the second image of Fig. 88. Also, a patch of light bluish grey can be found in the middle of the orange table cloth.

Most of the works of art mentioned above being either by one of or by painters later than the Impressionists, it is desirable to add here an oil painting from a considerably earlier period: Portrait of a Woman at the Spinning Wheel (reproduced in Artwork Fig. 10), by the 16thcentury Dutch painter Maerten van Heemskerck. In it, there are two white cloth bands hanging down from the woman's white hood; of primary interest is the right one, part of which is in front of her left shoulder. Being fairly thick, the band, lit by light from the right, has a long thin dark side on the left of it. Yet noticeably this dark side is not at all of the same colour throughout: it is almost pitch-black where its background is the well-lit brown wall, but rather light-coloured where its background is the woman's black garment. This pattern is practically the same as that in the simultaneous contrast illusion, discussed much earlier (Fig. 23-25), except that in this painting the colour of the background in question changes abruptly from black to brown, rather than smoothly. Rendered in this way, this band, in particular its upper part against the wall, appears aesthetically appealing, and in fact significantly more so than if its dark side had been everywhere painted with the same colour (namely, a dark grey in accordance with photographic truth), regardless of the marked shift in brightness in the background.

\section{Additional remarks on colour visual filtering}

As a supplement to the preceding three sections, a couple of questions are raised and answered. The first one: What if an already filtered image is filtered again? In other words, is it going to look even better? Not if the filter used is good enough; the effects will probably look overdone. Two or more visual filterings of an image with the same filter is equivalent to a single filtering with a new filter that has the same peak position as, but has a horizontally narrower shape (namely, is thinner) than, the original filter. The second one: What if an excellent oil painting, as by a master, is filtered? Is it going to be improved aesthetically? No. If the painting is thought of analogously as a table (or alternatively a chair) of high quality, 
then the aspect of the artwork for which visual filtering is responsible, is, correspondingly, one of the legs of the table, which together firmly support it. If this leg is lengthened, the table will thereby, in a rather peculiar manner, be damaged. There is no chance that the computer, by doing visual filtering, will make changes on the painting that surpass the aesthetic judgement of a fully qualified painter.

Very complex of course art is, and naturally what Fourier visual filtering reveals can only be an aspect of it, and a tentative step towards a better understanding of it and the visual system. Thus one cannot expect to create a work of art by using visual filtering alone; nor should one expect to form expert opinions or judgement of paintings solely from this point of view.

\section{More observations in relation to fine art}

Art is not only art: it is created by and for the human visual system and the human brain, and, scientifically speaking, about them it must tell a lot in return, of which too little is known yet. This paper may, it is hoped, have given a small amount of evidence for it. Conversely, a deeper knowledge of human vision is likely to improve the understanding of art. To this view can be added the following paragraph.

Western art of painting and traditional Chinese art of painting and calligraphy rely most heavily on, and have deeply explored, these two important function domains of human vision respectively: raster processing of visual information and vector processing of visual information. (As explained earlier, by vector is meant 'having direction and sensitive to shape and geometry', and by raster the opposite.) Hence the following traits in them, at once parallel and distinct, are readily understandable: the aesthetic value of an oil painting by a master is highly sensitive to the colours (which here include light and shade) and the combinations thereof used in it; and the aesthetic value of a masterpiece of Chinese calligraphy is highly sensitive to the shapes and relative positions of the strokes. Thus, for example, the difference in quality is colossal even between a single stroke by a master of calligraphy and the same stroke by a learner of calligraphy which looks, to the layman, remarkably similar to the master's; comparably, minute discrepancies in colour between a reproduction of a master's painting and that original painting can generally severely reduce the artistic value or beauty of the reproduced painting.

Brightness and colour illusion patterns found represented in oil painting are the more direct evidence of the relations between art and visual illusions, while the connection between geometric, or vector, illusions and Chinese painting and calligraphy is less clear.

Painters in oil, either today or in the past, have not seemed to be unanimously aware, however, that depicting their visual illusions is a thing of great import that they always do, although they generally know that certain very obvious phenomena that they see of colour contrast between two adjacent colours one of which is large, saturated, and vivid are visual illusions. A crucial thing to painting that they do with close attention, by training, is to look for on the objects from life what can be called the 'conditional', or 'imparted', colours, and then depict them on the canvas, those two terms referring herein to the same thing.

Colours are 'imparted' to an object, through physical, rather than mental, processes, by (1) the light source, if the light from it is not completely white, and (2) the object's physical 
environment, namely those other objects which are beside or around that object. For example, suppose a real scene is constructed according to the one in the left image of Fig. 88 . If this real scene is illuminated by a light source that is moderately yellow, as from an incandescent lamp, then all the objects in the scene, including of course the ground and the wall, will be somewhat 'dyed' by this yellow light and bear a tint of yellow; in other words, the light source imparts its yellow colour to the objects in the scene. Also, the objects in the scene can usually, to a small extent, impart their own colours to each other by means of reflection. For example, since the ground and the wall are greyish-blue, light reflected from them onto the cubes is greyish-blue and will therefore impart this colour to the cubes; the red cube too can likewise impart its red colour slightly onto the green cylinder as well as the ground, and so forth. Such imparted colours enhance the unity and harmony of the scene and hence are of great value and special importance to the painter.

Thus when the painter is looking at the objects of a scene from life, he or she is sensing not only the physically imparted colours but also colours that are in fact colour illusions created by his or her visual system; but traditionally they think that in most cases what they see is simply the physically imparted colours. What is more, even if one knows that visual illusion is taking part in one's observation while one is painting from life, it is often impossible for one to tell, as when a tint of greyish-blue is seen on the red cube, how much of that tint is physically real and how much is visual illusion. Nevertheless, in certain circumstances (as with the artworks collectively discussed above), it is still possible to decide with certainty that what is represented is, or mainly is, visual illusion.

Why then have artists often depicted their visual illusions? As regards the art of oil painting, the answer is as follows.

In artistic terms, the concise answer is twofold: first, the illusions are what the traditional artist sees, either with the eye or, often, with the mind's eye; and, second, consolidating or reasonably emphasizing the illusions with paint on the canvas is necessary for producing an aesthetically superior painting.

In more detail, the answer that the author attempts to give is this. The visual illusion formed of a visual scene helps to produce in the brain a more unified (and, in this sense, simpler) and more beautiful representation of that scene; in other words the illusion is, as mentioned before, aesthetically superior to the real scene. Nevertheless, in order to form that more unified and more beautiful representation, the brain has to do work and use energy, and would in fact do more and use more than if the visual scene being looked at were replaced by the illusionary form of the original scene. Being more unified, however, is not equivalent to being more monotonous or having fewer details, but to being more harmonious, having simpler mechanisms, and possessing more unity. For example, of the two images in Fig. 88, the filtered one on the right is the more unified, although it has more details in colour, and appears to be more complicated, than the original one on the left; those new details have by no means been added randomly, but to give more order and more unity to the original image. The same goes for other original-and-filtered image pairs. So it is with enough variations (for example in colour) that the illusionary representation is more unified than the original scene, and in those variations the eye and brain can play, free from fatigue.

It must be made very clear, however, that depiction of such visual illusions as have been accounted for by Fourier visual filtering is just one of the aesthetically significant ingredients 
of oil painting, there being other ones as well of no less importance. Yet, by standards of science, what the others are is still unknown.

\section{References}

1. De Valois, R.L., Morgan, H., \& Snodderly, D.M. Psychophysical studies of monkey vision. 3. Spatial luminance contrast sensitivity tests of macaque and human observers. Vision Res 14, 75-81. (1974).

2. Speed, H. Oil Painting Techniques and Materials (Dover Publications, 1987).

3. Geier, J., Bernáth, L., Hudák, M., \& Séra, L. Straightness as the main factor of the Hermann grid illusion. Perception 37, 651-65. (2008).

4. Hubel, D.H. \& Wiesel, T.N. (1959) Receptive fields of single neurons in the cat's striate cortex. Journal of Physiology, 148, 574-591.

5. Schrauf, M., Lingelbach, B., \& Wist, E.R. The scintillating grid illusion. Vision Research 37, 1033-8. (1997).

\section{Artwork figures}




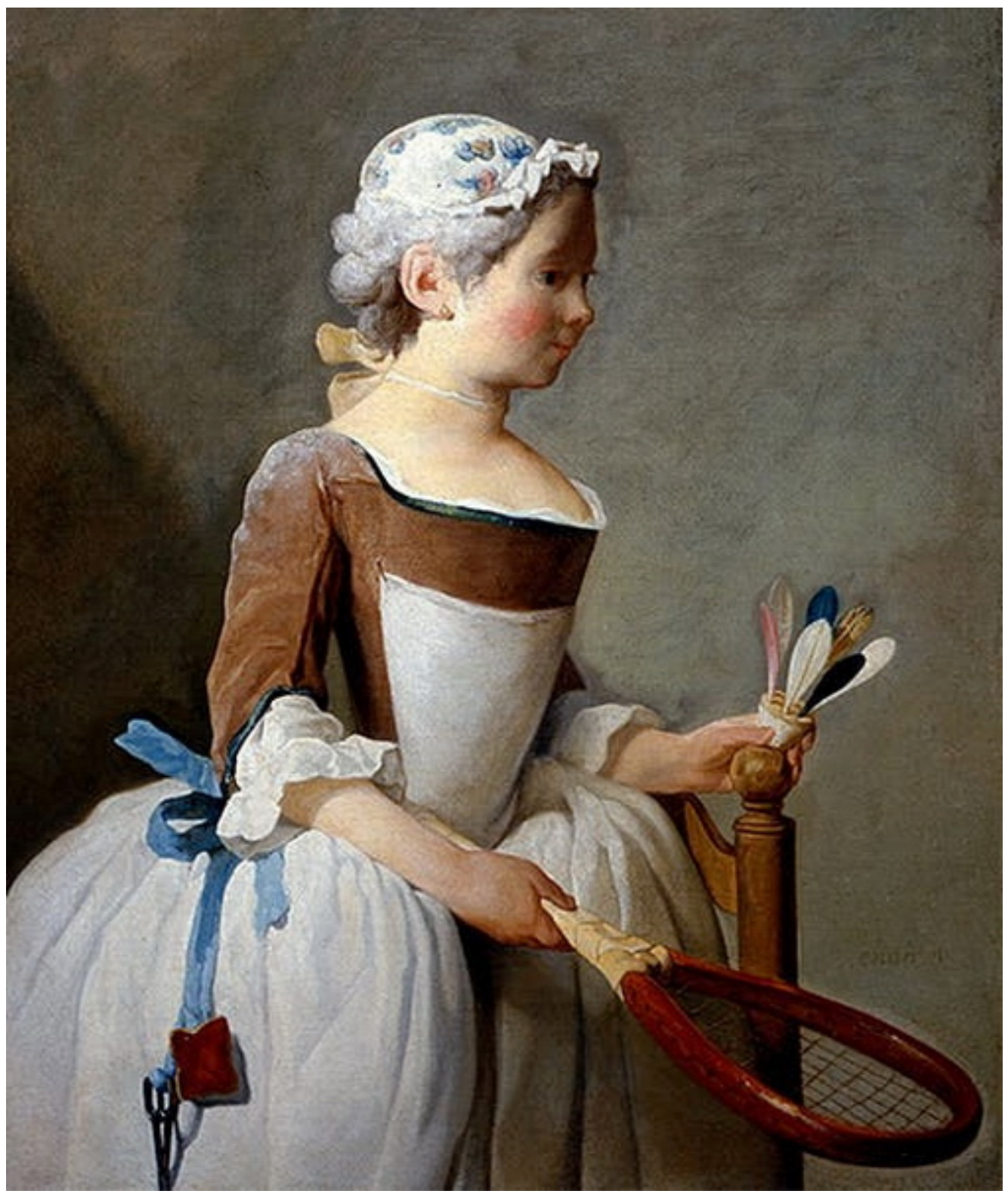

Artwork Figure 1 


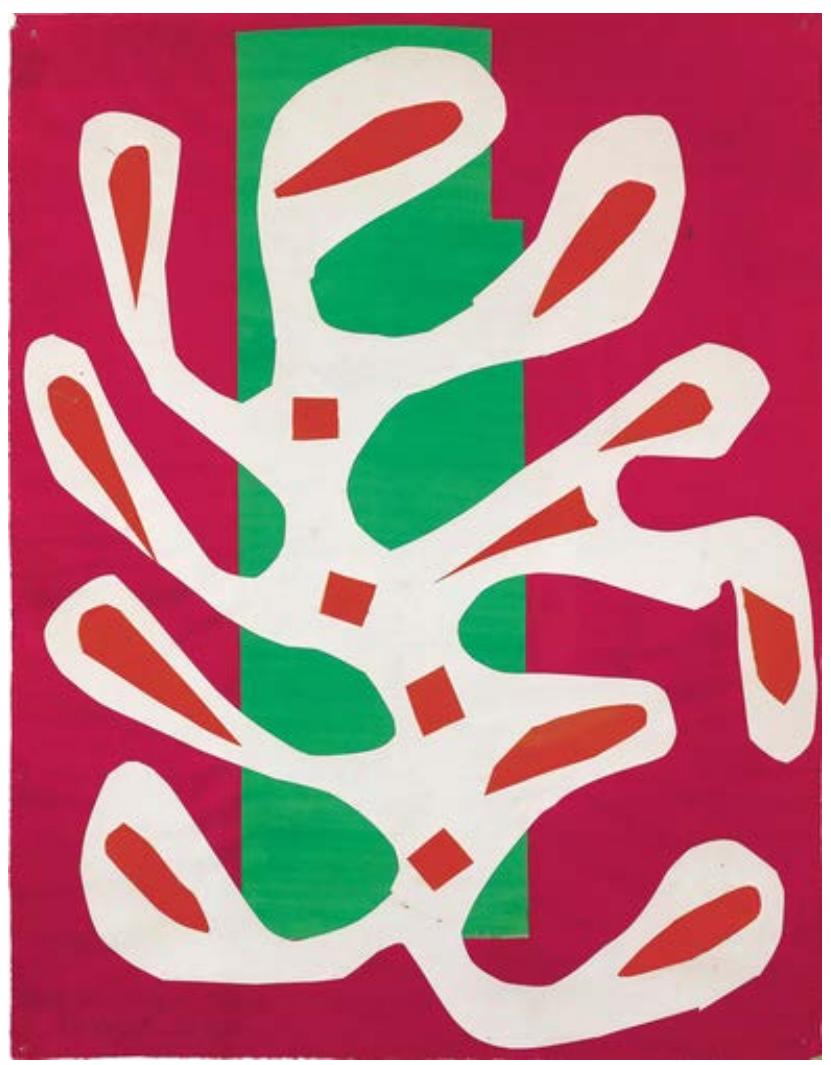

Artwork Figure 2

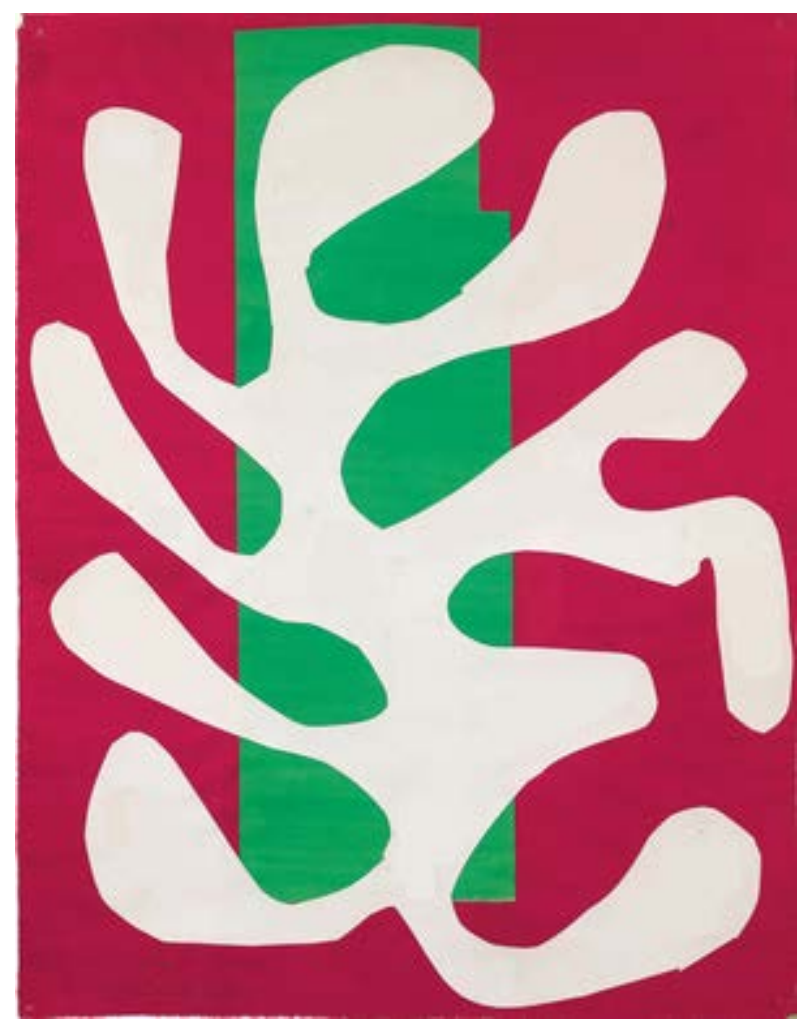

Artwork Figure 3 


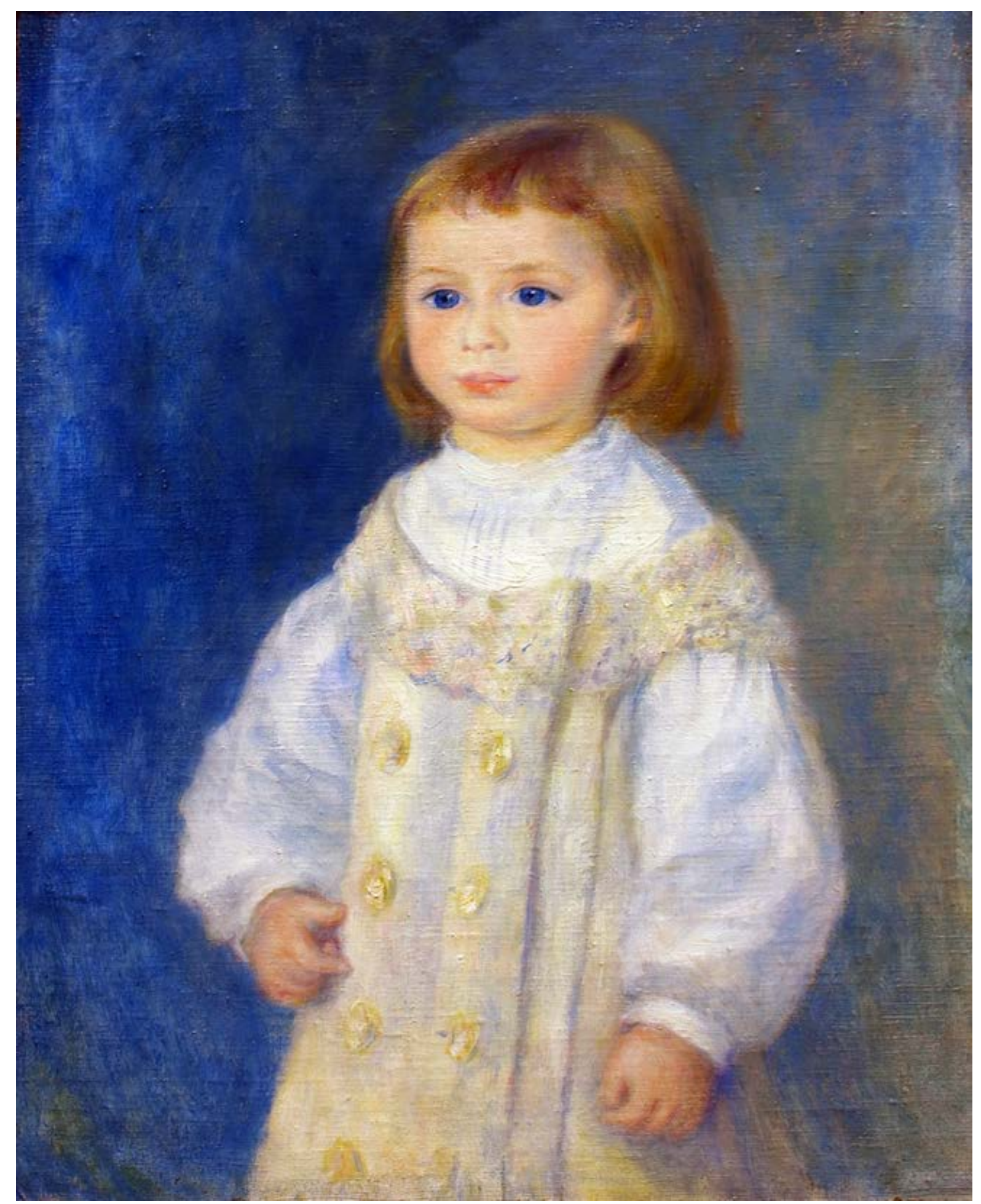

Artwork Figure 4 


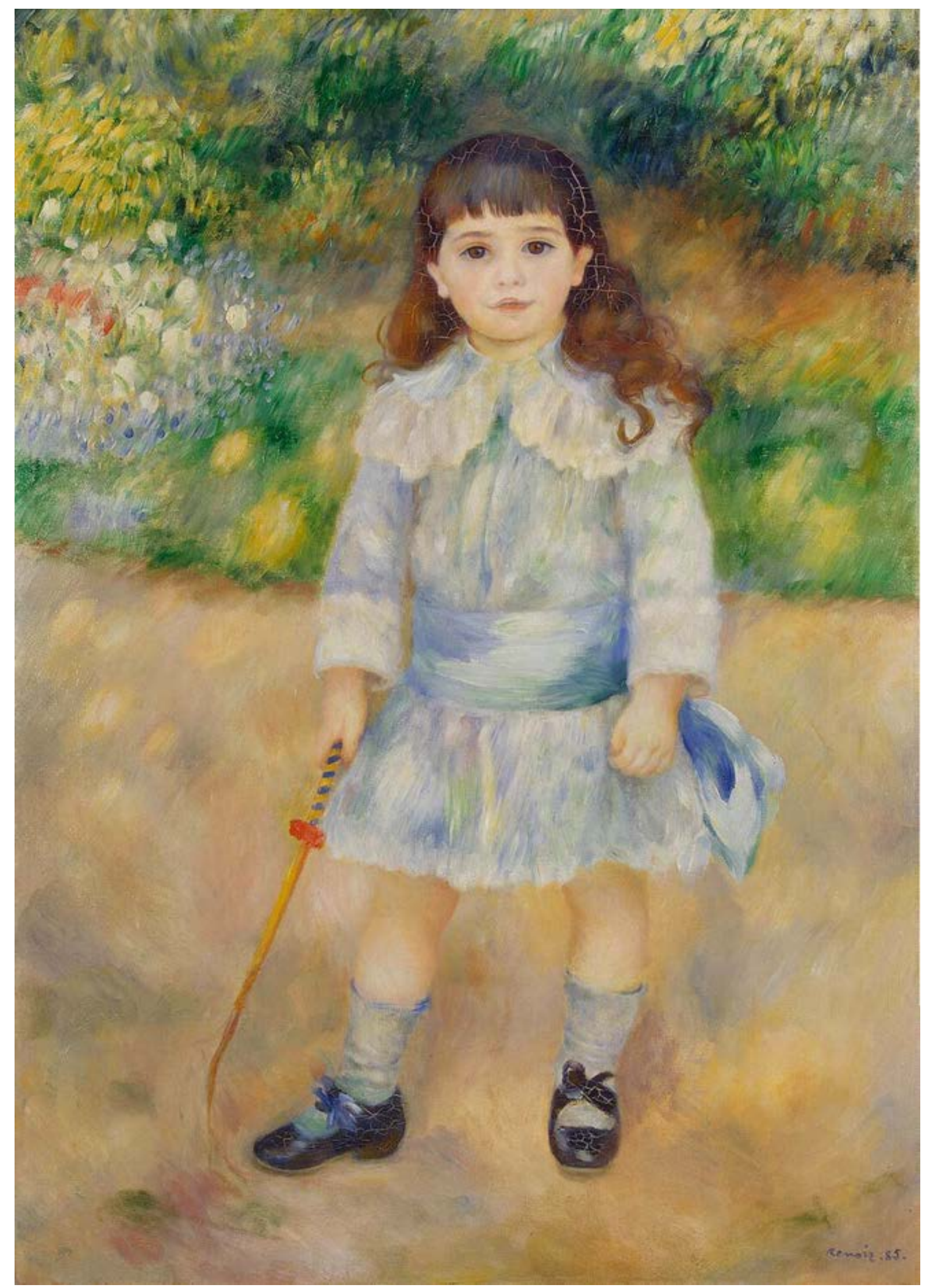

Artwork Figure 5 


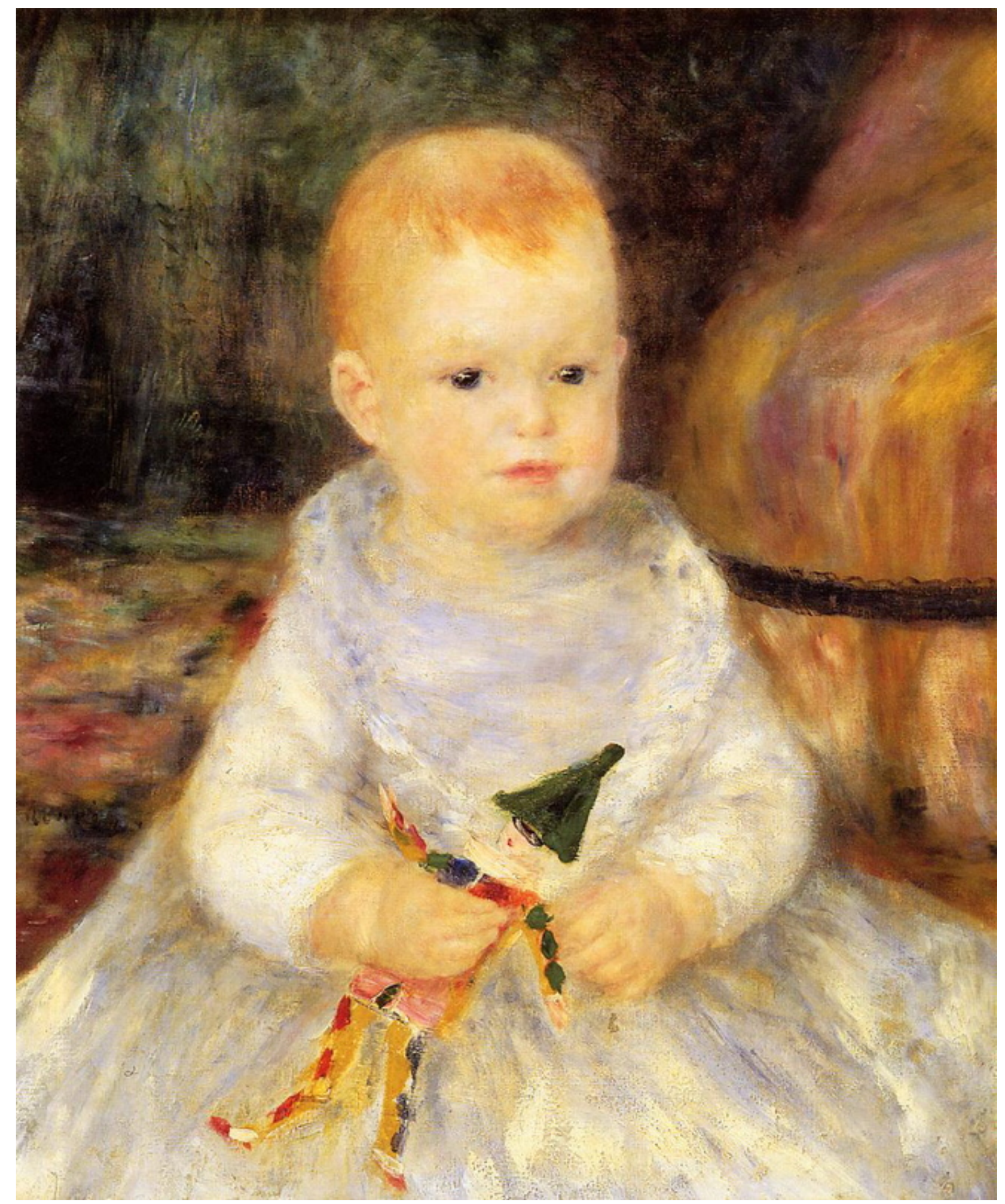

Artwork Figure 6 


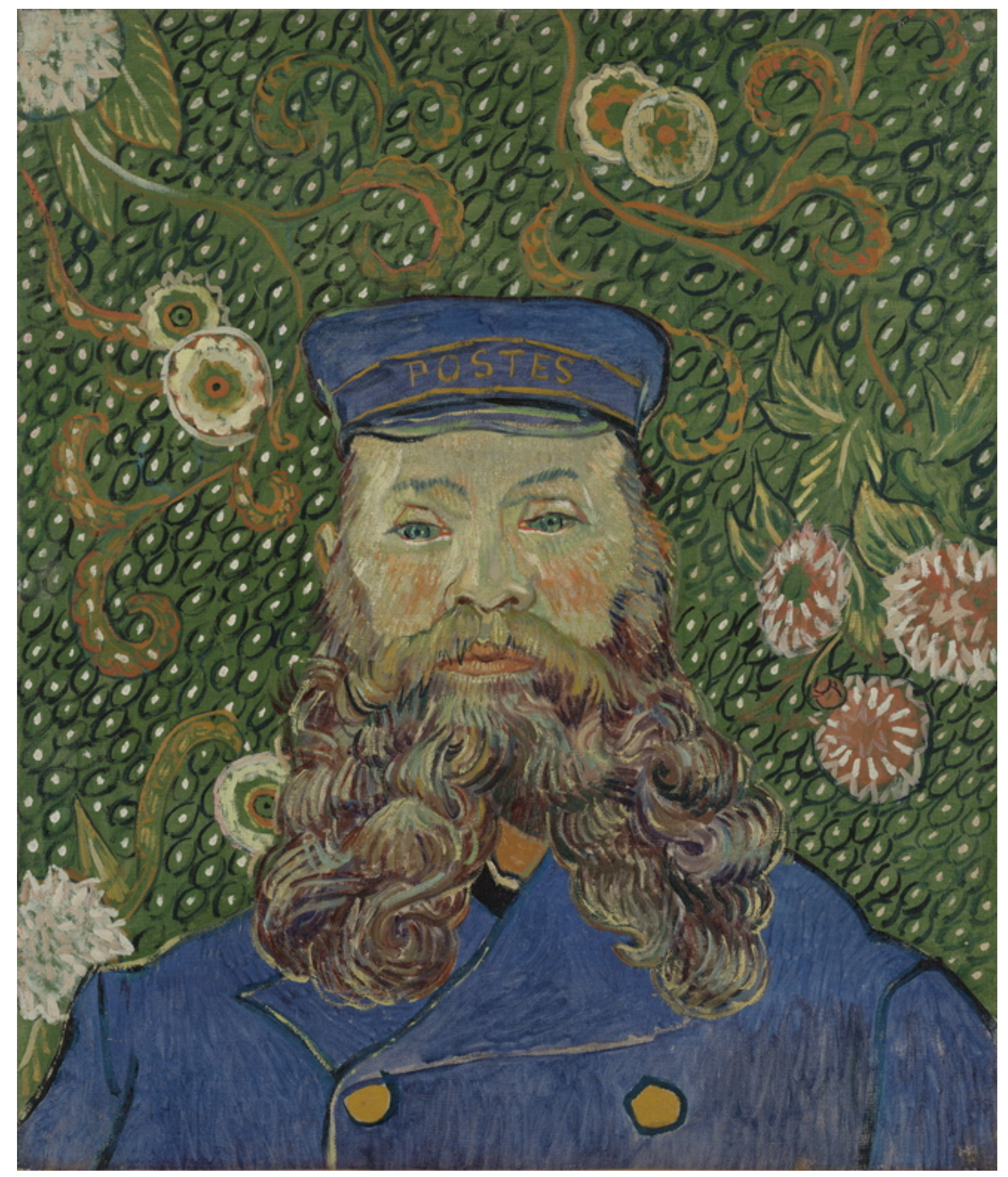

Artwork Figure 7 


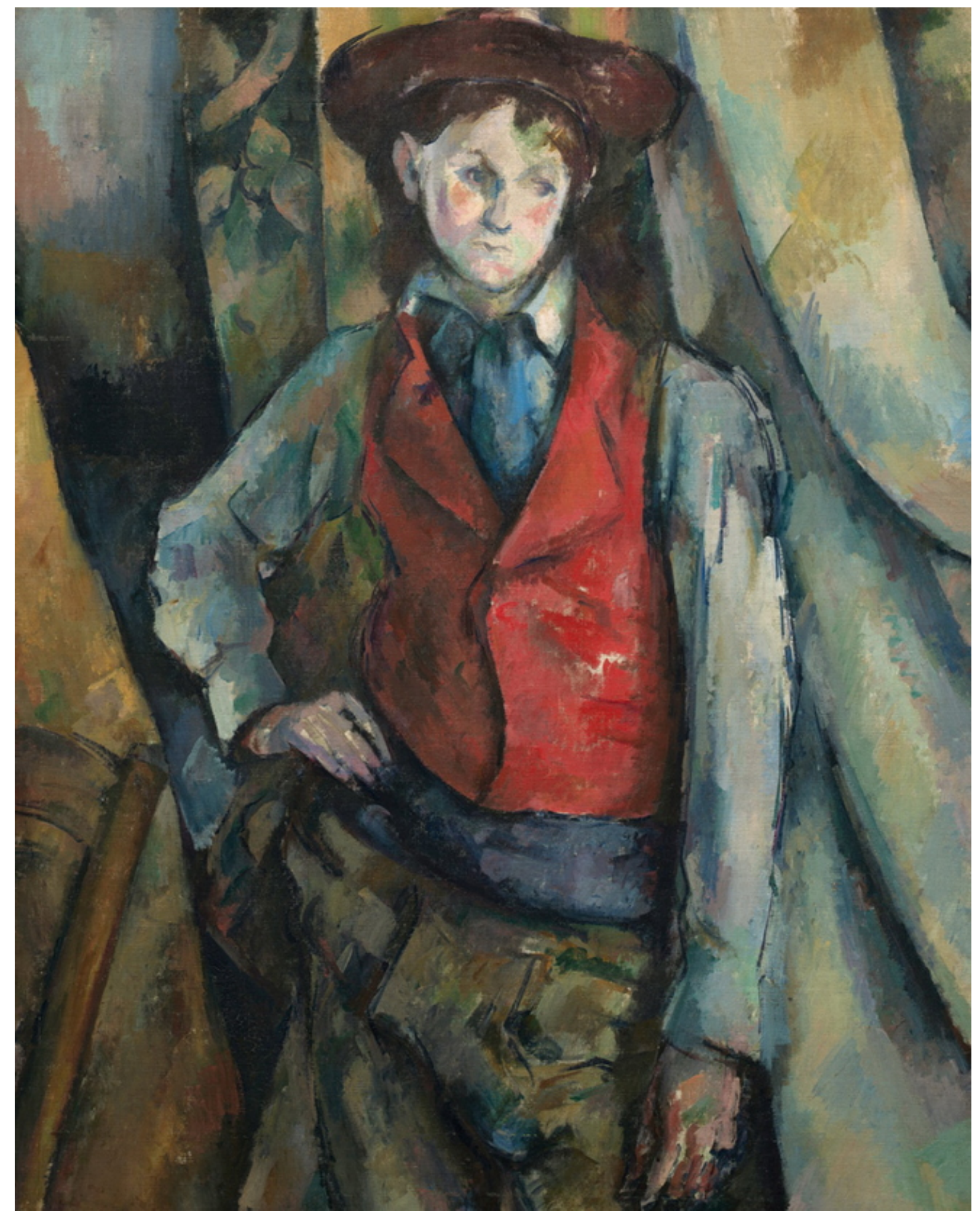

Artwork Figure 8 


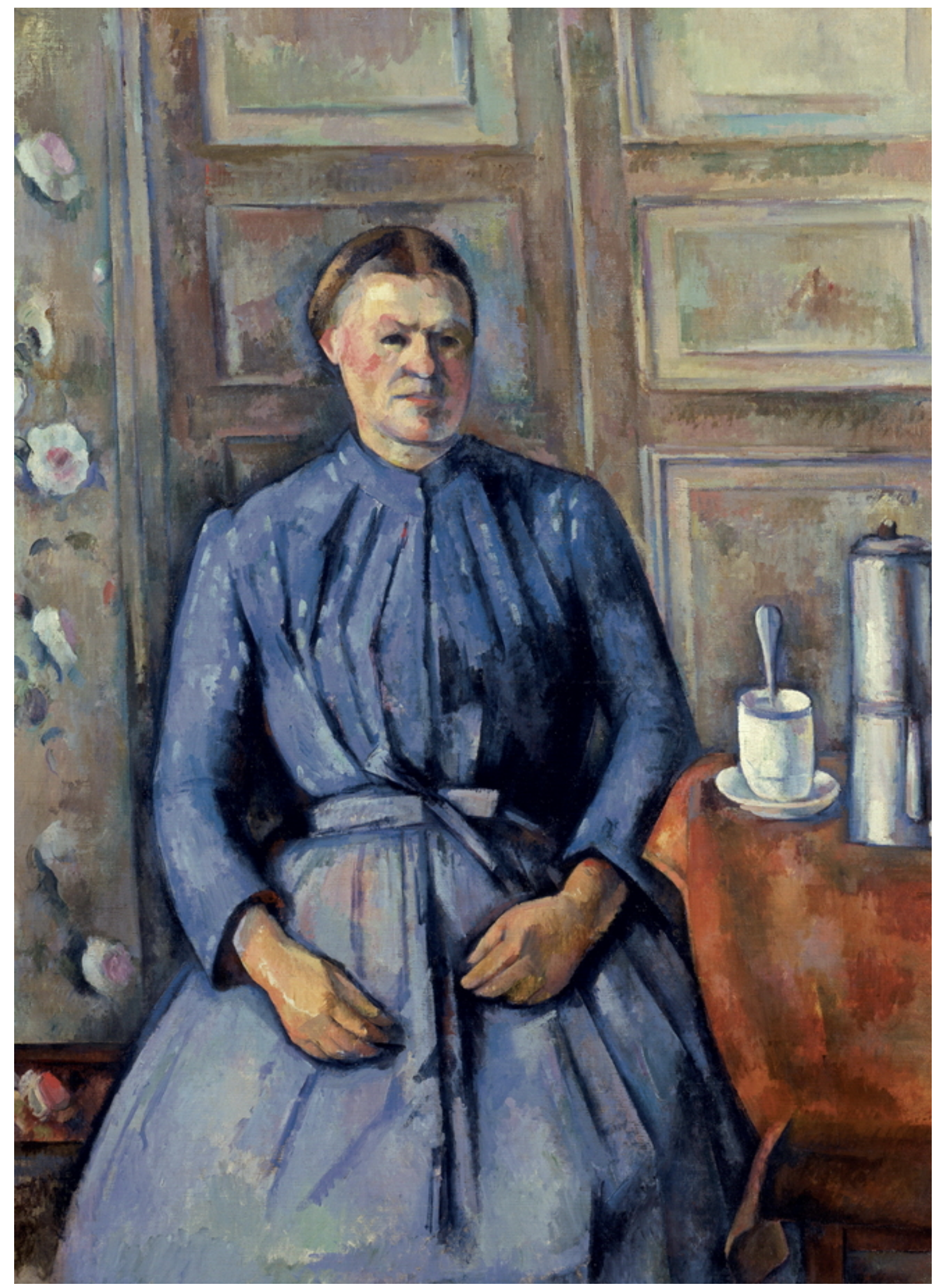

Artwork Figure 9 


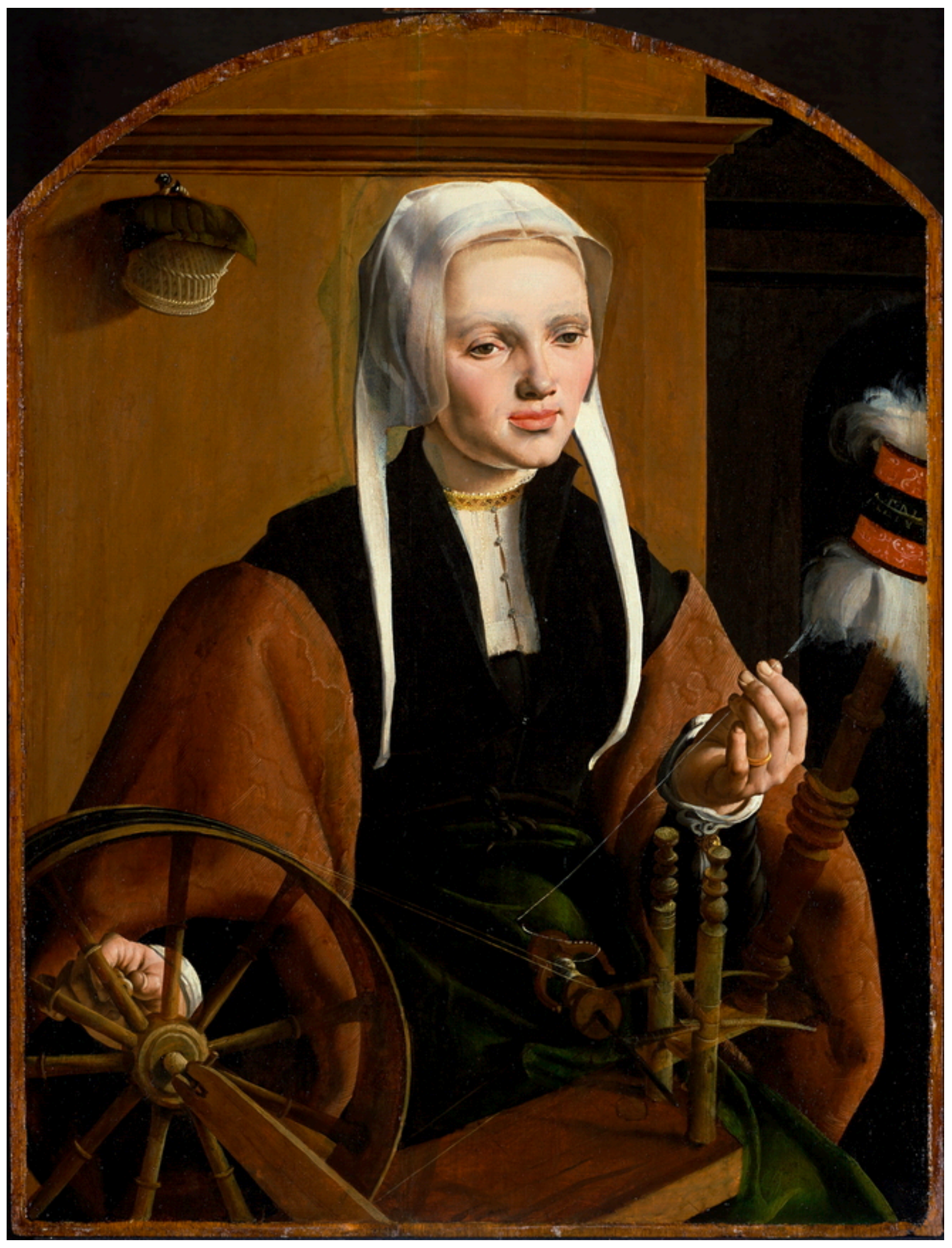

Artwork Figure 10 